\title{
RESIDUAL-BASED A POSTERIORI ERROR ANALYSIS FOR THE COUPLING OF THE NAVIER-STOKES AND DARCY-FORCHHEIMER EQUATIONS
}

\author{
Sergio Caucho ${ }^{1, *}$, Gabriel N. Gatica ${ }^{2}$, \\ Ricardo Oyarzúa ${ }^{2,3}$ AND Felipe SANDOvaL ${ }^{2}$
}

\begin{abstract}
In this paper we consider a mixed variational formulation that have been recently proposed for the coupling of the Navier-Stokes and Darcy-Forchheimer equations, and derive, though in a non-standard sense, a reliable and efficient residual-based a posteriori error estimator suitable for an adaptive mesh-refinement method. For the reliability estimate, which holds with respect to the square root of the error estimator, we make use of the inf-sup condition and the strict monotonicity of the operators involved, a suitable Helmholtz decomposition in non-standard Banach spaces in the porous medium, local approximation properties of the Clément interpolant and Raviart-Thomas operator, and a smallness assumption on the data. In turn, inverse inequalities, the localization technique based on triangle-bubble and edge-bubble functions in local $L^{p}$ spaces, are the main tools for developing the efficiency analysis, which is valid for the error estimator itself up to a suitable additional error term. Finally, several numerical results confirming the properties of the estimator and illustrating the performance of the associated adaptive algorithm are reported.
\end{abstract}

Mathematics Subject Classification. 65N30, 65N12, 65N15, 35Q79, 80A20, 76R05, 76D07.

Received April 20, 2020. Accepted January 25, 2021.

\section{INTRODUCTION}

We have recently introduced in [11] a primal-mixed finite element method to numerically approximate the fluid flow between porous media and free-flow zones described by the coupling of the Navier-Stokes and DarcyForchheimer equations together with mass conservation, balance of normal forces, and the Beavers--JosephSaffman condition on the interface. More precisely, a primal-mixed variational formulation was derived and analyzed in [11], which consists in employing the standard velocity-pressure mixed formulation in the NavierStokes domain and the dual-mixed one in the Darcy-Forchheimer region, which yields the introduction of the trace of the porous medium pressure as a suitable Lagrange multiplier. The well-posedness of the problem is achieved by combining a fixed-point strategy, classical results on nonlinear monotone operators and the

Keywords and phrases. Navier-Stokes problem, Darcy-Forchheimer problem, primal-mixed finite element methods, a posteriori error analysis.

1 Departamento de Matemática y Física Aplicadas, Universidad Católica de la Santísima Concepción, Casilla 297, Concepción, Chile.

${ }^{2} \mathrm{CI}^{2} \mathrm{MA}$ and Departamento de Ingeniería Matemática, Universidad de Concepción, Casilla 160-C, Concepción, Chile

3 GIMNAP-Departamento de Matemática, Universidad del Bío-Bío, Casilla 5-C, Concepción and $\mathrm{CI}^{2} \mathrm{MA}$, Universidad de Concepción, Casilla 160-C, Concepción, Chile.

*Corresponding author: scaucao@ucsc.cl 
well-known Schauder and Banach fixed-point theorems. A feasible choice of finite element subspaces for the formulation introduced in [11] is given by Bernardi-Raugel and Raviart-Thomas elements for the velocities, and piecewise constant elements for the pressures and the Lagrange multiplier. Sub-optimal a priori error estimates were also derived.

Now, it is well known that under the eventual presence of singularities, as well as when dealing with nonlinear problems, as in the present case, most of the standard Galerkin procedures such as finite element and mixed finite element methods inevitably lose accuracy, and hence one usually tries to recover it by applying an adaptive algorithm based on a posteriori error estimates. For example, residual-based a posteriori error analyses for the Stokes-Darcy and Navier-Stokes/Darcy coupled problems have been developed in $[4,9]$ for the associated primalmixed and fully-mixed formulations, respectively. In fact, standard arguments relying on duality techniques, suitable decompositions and classical approximation properties, are combined there with corresponding small data assumptions to derive the reliability of the estimators. In turn, inverse inequalities and the usual localization technique based on bubble functions are employed in both works to prove the corresponding efficiency estimates. Now, concerning the Navier-Stokes problem, we begin by referring to [29,31], where the authors develop one of the first a posteriori error analyses for the incompressible Navier-Stokes problem in its classical velocitypressure formulation. Interestingly, several terms that are derived in Section 5.4 of [31] will also appear in the estimator to be introduced in the present paper. Other recent and not so recent contributions on a posteriori error estimates for Navier-Stokes and related models include, e.g. $[2,15,19,23,28]$. In particular, the evolutionary problem is considered in [15] and a Newton-type linearization is employed there to reduce the corresponding a posteriori error analysis to that of a linear steady problem. In addition, the stationary model with singular sources is studied in [2], whereas a dual mixed-formulation and exactly divergence-free discontinuous Galerkin methods are employed in [19,28], respectively. Furthermore, an a posteriori error analysis for an augmented mixed formulation of the Navier-Stokes-Brinkman problem is developed in [23]. On the other hand, for quasiNewtonian fluid flows obeying to the power law, as in the case of the Darcy-Forchheimer model, not much has been done and we just refer to $[13,17,18]$, where different contributions addressing this interesting issue can be found. In particular, an a posteriori error estimator defined via a non-linear projection of the residues of the variational equations for a three-field model of a generalized Stokes problem was proposed and analyzed in [17]. We remark that the non-linear projections do not need to be explicitly computed to construct the a posteriori error estimates. In turn, a fully local residual-based a posteriori error estimator for the mixed formulation of the $p$-Laplacian problem in a polygonal domain, was derived in [13]. In this case, the authors study the reliability of the estimator defining two residues and then bounding the norm of the errors in terms of the norms of these residues. Moreover, the discretized dual-mixed formulation is hybridized and it is provided several tests for $p=1.8$ and $p=3$ to experimentally verify the reliability of the estimator. We remark that up to the authors' knowledge, there are no works dealing with the a posteriori error analysis for the coupling of the Navier-Stokes (or Stokes) and the Darcy-Forchheimer models.

According to the above discussion and aiming to complement previous results on the numerical analysis of the coupled Navier-Stokes and Darcy-Forchheimer equations, in this paper we proceed similarly to $[4,13,17,18,21,22]$ and [9], and develop a residual-based a posteriori error estimator, say $\Theta$, for the primal-mixed finite element method introduced and analyzed in [11]. Due to the nonlinear nature of the coupled problem, $\Theta^{1 / 2}$ is shown to be reliable, whereas $\Theta$ itself becomes efficient up to the error associated to a lifting of the trace of the porous medium pressure, which we name "lifting-error". More precisely, we basically prove that there exist positive constants $C_{\text {eff }}$ and $C_{\mathrm{rel}}$, independent of the meshsizes, such that

$$
\| \text { error } \| \leq C_{\text {rel }} \Theta^{1 / 2}, \quad \text { and } \quad C_{\text {eff }} \Theta \leq \| \text { error }\|+\| \text { lifting-error } \|+ \text { h.o.t., }
$$

where h.o.t. is a generic expression denoting one or several terms of higher order. Indeed, starting from the inf-sup condition and the strict monotonicity of the operators involved, and employing suitable Helmholtz decompositions in non-standard Banach spaces, we prove the reliability of the estimator under a smallness condition on the data. In turn, the efficiency estimate is consequence of standard arguments such as inverse inequalities and the localization technique based on triangle-bubble and edge-bubble functions in local $L^{p}$ spaces. 
The rest of this work is organized as follows. In the remainder of this section we introduce some standard notations and definitions of functional spaces. In Section 2 we recall from [11] the model problem. Next, in Section 3 we describe the continuous formulation and the corresponding primal-mixed finite element method, whereas some preliminary results, necessary to the a posteriori error analysis, are established in Section 4 . Then, in Section 5 we introduce an a posteriori error indicator and, assuming small data, we derive the corresponding theoretical bounds yielding reliability and efficiency of the estimator in the sense of (1.1). Finally, some numerical results confirming the theoretical sub-optimal order of convergence, and at the same time suggesting an optimal rate of convergence as in [11], are presented in Section 6. Additionally, these numerical essays illustrate the efficiency and reliability of the a posteriori error estimator, and show the good performance of the associated adaptive algorithm for the finite element method.

We end this section by introducing some definitions and fixing some notations. Let $\mathcal{O} \subseteq \mathbb{R}^{2}$ be a domain with Lipschitz-continuous boundary $\Gamma$. For $s \geq 0$ and $p \in[1,+\infty]$ we denote by $L^{p}(\mathcal{O})$ and $W^{s, p}(\mathcal{O})$ the usual Lebesgue and Sobolev spaces endowed with the norms $\|\cdot\|_{L^{p}(\mathcal{O})}$ and $\|\cdot\|_{s, p ; \mathcal{O}}$, respectively. Also, we let $|\cdot|_{s, p ; \mathcal{O}}$ be the seminorm of $W^{s, p}(\mathcal{O})$. Note that $W^{0, p}(\mathcal{O})=L^{p}(\mathcal{O})$. In turn, when $p=2$, we write $H^{s}(\mathcal{O})$ in place of $W^{s, 2}(\mathcal{O})$, and denote the corresponding Lebesgue and Sobolev norms by $\|\cdot\|_{0, \mathcal{O}}$ and $\|\cdot\|_{s, \mathcal{O}}$, respectively, and the seminorm by $|\cdot|_{s, \mathcal{O}}$. In addition, we denote by $W^{1 / q, p}(\Gamma)$ the trace space of $W^{1, p}(\mathcal{O})$, and let $W^{-1 / q, q}(\Gamma)$ be the dual space of $W^{1 / q, p}(\Gamma)$ endowed with the norms $\|\cdot\|_{1 / q, p ; \Gamma}$ and $\|\cdot\|_{-1 / q, q ; \Gamma}$, respectively, with $p, q \in(1,+\infty)$ satisfying $1 / p+1 / q=1$. By $\mathbf{M}$ and $\mathbb{M}$ we will denote the corresponding vectorial and tensorial counterparts of a generic scalar functional space $M$, and $\|\cdot\|$, with no subscripts, will stand for the natural norm of either an element or an operator in any product functional space. Additionally, we recall that

$$
\mathbf{H}(\operatorname{div} ; \mathcal{O}):=\left\{\mathbf{w} \in \mathbf{L}^{2}(\mathcal{O}): \quad \operatorname{div} \mathbf{w} \in L^{2}(\mathcal{O})\right\}
$$

is a standard Hilbert space in the realm of mixed problems (see, e.g. [5]). On the other hand, the following symbol for the $L^{2}(\Gamma)$ inner product

$$
\langle\xi, \lambda\rangle_{\Gamma}:=\int_{\Gamma} \xi \lambda \quad \forall \xi, \lambda \in L^{2}(\Gamma)
$$

will also be employed for their respective extension as the duality parity between $W^{-1 / q, q}(\Gamma)$ and $W^{1 / q, p}(\Gamma)$. Hereafter, when no confusion arises, $|\cdot|$ will denote the Euclidean norm in $\mathbb{R}^{2}$ or $\mathbb{R}^{2 \times 2}$. Furthermore, given a non-negative integer $k$ and a subset $S$ of $\mathbb{R}^{2}, P_{k}(S)$ denotes the space of polynomial functions on $S$ of degree $\leq k$. In addition, and coherently with previous notations, we set

$$
\mathbf{P}_{k}(S):=\left[P_{k}(S)\right]^{2} \quad \text { and } \quad \mathbb{P}_{k}(S):=\left[P_{k}(S)\right]^{2 \times 2} .
$$

Finally, we employ $\mathbf{0}$ as a generic null vector, and use $C$ and $c$, with or without subscripts, bars, tildes or hats, to denote generic positive constants independent of the discretization parameters, which may take different values at different places.

\section{THE MODEL PROBLEM}

In order to describe the geometry under consideration we let $\Omega_{S}$ and $\Omega_{D}$ be two bounded and simply connected polygonal domains in $\mathbb{R}^{2}$ such that $\partial \Omega_{S} \cap \partial \Omega_{D}=\Sigma \neq \emptyset$ and $\Omega_{S} \cap \Omega_{D}=\emptyset$. Then, let $\Gamma_{S}:=\partial \Omega_{S} \backslash \bar{\Sigma}$, $\Gamma_{D}:=\partial \Omega_{D} \backslash \bar{\Sigma}$, and denote by $\mathbf{n}$ the unit normal vector on the boundaries, which is chosen pointing outward from $\Omega:=\Omega_{S} \cup \Sigma \cup \Omega_{D}$ and $\Omega_{S}$ (and hence inward to $\Omega_{D}$ when seen on $\Sigma$ ). On $\Sigma$ we also consider a unit tangent vector $\mathbf{t}$ (see Fig. 1).

The problem we are interested in consists of the movement of an incompressible viscous fluid occupying $\Omega_{S}$ which flows towards and from a porous medium $\Omega_{D}$ through $\Sigma$, where $\Omega_{D}$ is saturated with the same fluid. The 


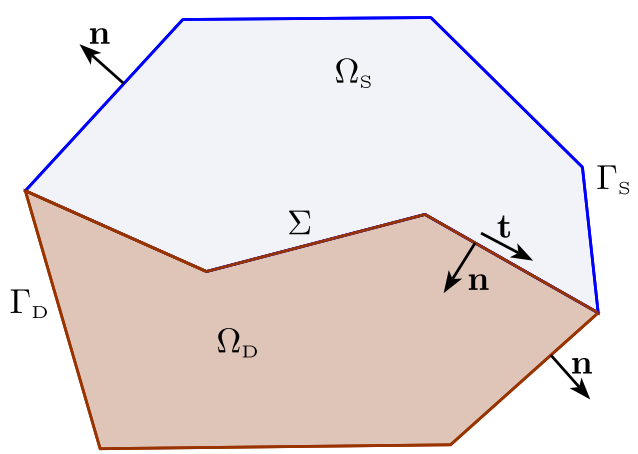

FiguRE 1. Sketch of a 2D geometry of our Navier-Stokes/Darcy-Forchheimer model.

mathematical model is defined by two separate groups of equations and by a set of coupling terms. In the free fluid domain $\Omega_{S}$, the motion of the fluid can be described by the incompressible Navier-Stokes equations:

$$
\begin{array}{llll}
\boldsymbol{\sigma}_{S}=-p_{S} \mathbb{I}+2 \mu \mathbf{e}\left(\mathbf{u}_{S}\right) & \text { in } \quad \Omega_{S}, & -\operatorname{div} \boldsymbol{\sigma}_{S}+\rho\left(\nabla \mathbf{u}_{S}\right) \mathbf{u}_{S}=\mathbf{f}_{S} \text { in } \Omega_{S}, \\
\operatorname{div} \mathbf{u}_{S}=0 \text { in } \Omega_{S}, & & \mathbf{u}_{S}=\mathbf{0} \text { on } \Gamma_{S},
\end{array}
$$

where the unknowns are the fluid velocity $\mathbf{u}_{S}$, the pressure $p_{S}$, and the Cauchy stress tensor $\boldsymbol{\sigma}_{S}$. In addition, $\mathbf{e}\left(\mathbf{u}_{S}\right):=\frac{1}{2}\left\{\nabla \mathbf{u}_{S}+\left(\nabla \mathbf{u}_{S}\right)^{t}\right\}$ stands for the strain tensor of small deformations, $\mu$ is the viscosity of the fluid, $\rho$ is the density, and $\mathbf{f}_{S} \in \mathbf{L}^{2}\left(\Omega_{S}\right)$ is a given external force.

On the other hand, as was explained in [11], given functions $\mathbf{f}_{D} \in \mathbf{L}^{3 / 2}\left(\Omega_{D}\right)$ and $g_{D} \in L^{2}\left(\Omega_{D}\right)$, in the porous medium $\Omega_{D}$ we consider the Darcy-Forchheimer equations to approximate the velocity $\mathbf{u}_{D}$ and the pressure $p_{D}:$

$$
\frac{\mu}{\rho} \mathbf{K}^{-1} \mathbf{u}_{D}+\frac{\mathrm{F}}{\rho}\left|\mathbf{u}_{D}\right| \mathbf{u}_{D}+\nabla p_{D}=\mathbf{f}_{D} \quad \text { in } \quad \Omega_{D}, \quad \operatorname{div} \mathbf{u}_{D}=g_{D} \quad \text { in } \quad \Omega_{D}, \quad \mathbf{u}_{D} \cdot \mathbf{n}=0 \quad \text { on } \quad \Gamma_{D},
$$

where $\mathbf{F}$ represents the Forchheimer number of the porous medium, and $\mathbf{K} \in \mathbb{L}^{\infty}\left(\Omega_{D}\right)$ is a symmetric tensor in $\Omega_{D}$ representing the intrinsic permeability $\boldsymbol{\kappa}$ of the porous medium divided by the viscosity $\mu$ of the fluid. We assume that there exists $C_{\mathbf{K}}>0$ such that $\mathbf{w} \cdot \mathbf{K}^{-1}(\mathbf{x}) \mathbf{w} \geq C_{\mathbf{K}}|\mathbf{w}|^{2}$, for almost all $\mathbf{x} \in \Omega_{D}$, and for all $\mathbf{w} \in \mathbb{R}^{2}$. In addition, according to the compressibility conditions, the boundary conditions on $\mathbf{u}_{D}$ and $\mathbf{u}_{S}$, and the principle of mass conservation ( $c f$. (2.3) below), $g_{D}$ must satisfy the compatibility condition:

$$
\int_{\Omega_{D}} g_{D}=0
$$

Finally, the transmission conditions that couple the Navier-Stokes and the Darcy-Forchheimer models through the interface $\Sigma$ are given by

$$
\mathbf{u}_{S} \cdot \mathbf{n}=\mathbf{u}_{D} \cdot \mathbf{n} \quad \text { on } \quad \Sigma \quad \text { and } \quad \boldsymbol{\sigma}_{S} \mathbf{n}+\frac{\alpha_{d} \mu}{\sqrt{\mathbf{t} \cdot \boldsymbol{\kappa} \mathbf{t}}}\left(\mathbf{u}_{S} \cdot \mathbf{t}\right) \mathbf{t}=-p_{D} \mathbf{n} \quad \text { on } \quad \Sigma,
$$

where $\alpha_{d}$ is a dimensionless positive constant which depends only on the geometrical characteristics of the porous medium and usually assumes values between 0.8 and 1.2. The first condition in (2.3) is a consequence of the incompressibility of the fluid and of the conservation of mass across $\Sigma$, whereas the second one establishes the balance of normal forces and a Beavers-Joseph-Saffman law.

\section{THE VARIATIONAL FORMULATION}

In this section we introduce the variational formulation for the coupling of the Navier-Stokes and DarcyForchheimer equations proposed in Section 2.2 of [11], and recall the respective solvability results. 


\subsection{Preliminaries}

We first introduce further notations and definitions. In what follows, given $\star \in\{S, D\}$, we set

$$
(u, v)_{\star}:=\int_{\Omega_{\star}} u v, \quad(\mathbf{u}, \mathbf{v})_{\star}:=\int_{\Omega_{\star}} \mathbf{u} \cdot \mathbf{v}, \quad \text { and } \quad(\boldsymbol{\sigma}, \boldsymbol{\tau})_{\star}:=\int_{\Omega_{\star}} \boldsymbol{\sigma}: \boldsymbol{\tau} .
$$

Furthermore, given $p \in[2,+\infty)$, in the sequel we will employ the following Banach space,

endowed with the norm

$$
\mathbf{H}^{p}\left(\operatorname{div} ; \Omega_{D}\right):=\left\{\mathbf{v}_{D} \in \mathbf{L}^{p}\left(\Omega_{D}\right): \quad \operatorname{div} \mathbf{v}_{D} \in L^{2}\left(\Omega_{D}\right)\right\},
$$

$$
\left\|\mathbf{v}_{D}\right\|_{\mathbf{H}^{p}\left(\operatorname{div} ; \Omega_{D}\right)}:=\left(\left\|\mathbf{v}_{D}\right\|_{\mathbf{L}^{p}\left(\Omega_{D}\right)}^{p}+\left\|\operatorname{div} \mathbf{v}_{D}\right\|_{0, \Omega_{D}}^{p}\right)^{1 / p}
$$

and the following subspaces of $\mathbf{H}^{p}\left(\operatorname{div} ; \Omega_{D}\right)$ and $\mathbf{H}^{1}\left(\Omega_{S}\right)$, respectively

$$
\begin{aligned}
\mathbf{H}_{\Gamma_{D}}^{p}\left(\operatorname{div} ; \Omega_{D}\right) & :=\left\{\begin{array}{llll}
\mathbf{v}_{D} \in \mathbf{H}^{p}\left(\operatorname{div} ; \Omega_{D}\right): & \mathbf{v}_{D} \cdot \mathbf{n}=0 \quad \text { on } \quad \Gamma_{D}
\end{array}\right\}, \\
H_{\Gamma_{S}}^{1}\left(\Omega_{S}\right) & :=\left\{\begin{array}{llll}
v_{S} \in H^{1}\left(\Omega_{S}\right): & v_{S}=0 & \text { on } & \Gamma_{S}
\end{array}\right\}, \quad \mathbf{H}_{\Gamma_{S}}^{1}\left(\Omega_{S}\right):=\left[H_{\Gamma_{S}}^{1}\left(\Omega_{S}\right)\right]^{2} .
\end{aligned}
$$

In addition, we write $\Omega:=\Omega_{S} \cup \Sigma \cup \Omega_{D}$, and define $p:=p_{S} \chi_{S}+p_{D} \chi_{D}$, with $\chi_{\star}$ being the characteristic function:

$$
\chi_{\star}:=\left\{\begin{array}{ll}
1 & \text { in } \Omega_{\star}, \\
0 & \text { in } \Omega \backslash \bar{\Omega}_{\star},
\end{array} \quad \text { for } \star \in \in S, D\right\},
$$

and introduce the space $L_{0}^{2}(\Omega):=\left\{q \in L^{2}(\Omega): \quad \int_{\Omega} q=0\right\}$.

\subsection{The continuous formulation}

Now, we introduce the weak formulation derived for the coupled problem given by (2.1)-(2.3) (see [11], Sect. 2.2 for details). In fact, we first group the spaces and unknowns as follows:

$$
\begin{array}{ll}
\mathbf{H}:=\mathbf{H}_{\Gamma_{S}}^{1}\left(\Omega_{S}\right) \times \mathbf{H}_{\Gamma_{D}}^{3}\left(\operatorname{div} ; \Omega_{D}\right), & \mathbf{Q}:=L_{0}^{2}(\Omega) \times W^{1 / 3,3 / 2}(\Sigma), \\
\mathbf{u}:=\left(\mathbf{u}_{S}, \mathbf{u}_{D}\right) \in \mathbf{H}, & (p, \lambda) \in \mathbf{Q},
\end{array}
$$

where $\lambda:=\left.p_{D}\right|_{\Sigma} \in W^{1 / 3,3 / 2}(\Sigma)$ is an additional unknown. Thus, we arrive at the mixed variational formulation: Find $(\mathbf{u},(p, \lambda)) \in \mathbf{H} \times \mathbf{Q}$, such that

$$
\begin{array}{ll}
{\left[\mathbf{a}\left(\mathbf{u}_{S}\right)(\mathbf{u}), \mathbf{v}\right]+[\mathbf{b}(\mathbf{v}),(p, \lambda)]=[\mathbf{f}, \mathbf{v}]} & \forall \mathbf{v}:=\left(\mathbf{v}_{S}, \mathbf{v}_{D}\right) \in \mathbf{H} \\
{[\mathbf{b}(\mathbf{u}),(q, \xi)]=[\mathbf{g},(q, \xi)]} & \forall(q, \xi) \in \mathbf{Q},
\end{array}
$$

where, given $\mathbf{w}_{S} \in \mathbf{H}_{\Gamma_{S}}^{1}\left(\Omega_{S}\right)$, the operator $\mathbf{a}\left(\mathbf{w}_{S}\right): \mathbf{H} \rightarrow \mathbf{H}^{\prime}$ is defined by

$$
\left[\mathbf{a}\left(\mathbf{w}_{S}\right)(\mathbf{u}), \mathbf{v}\right]:=\left[\mathcal{A}_{S}\left(\mathbf{u}_{S}\right), \mathbf{v}_{S}\right]+\left[\mathcal{B}_{S}\left(\mathbf{w}_{S}\right)\left(\mathbf{u}_{S}\right), \mathbf{v}_{S}\right]+\left[\mathcal{A}_{D}\left(\mathbf{u}_{D}\right), \mathbf{v}_{D}\right],
$$

with

$$
\begin{aligned}
{\left[\mathcal{A}_{S}\left(\mathbf{u}_{S}\right), \mathbf{v}_{S}\right] } & :=2 \mu\left(\mathbf{e}\left(\mathbf{u}_{S}\right), \mathbf{e}\left(\mathbf{v}_{S}\right)\right)_{S}+\left\langle\frac{\alpha_{d} \mu}{\sqrt{\mathbf{t} \cdot \boldsymbol{\kappa} \mathbf{t}}} \mathbf{u}_{S} \cdot \mathbf{t}, \mathbf{v}_{S} \cdot \mathbf{t}\right\rangle_{\Sigma} \\
{\left[\mathcal{B}_{S}\left(\mathbf{w}_{S}\right)\left(\mathbf{u}_{S}\right), \mathbf{v}_{S}\right] } & :=\rho\left(\left(\nabla \mathbf{u}_{S}\right) \mathbf{w}_{S}, \mathbf{v}_{S}\right)_{S}, \\
{\left[\mathcal{A}_{D}\left(\mathbf{u}_{D}\right), \mathbf{v}_{D}\right] } & :=\frac{\mu}{\rho}\left(\mathbf{K}^{-1} \mathbf{u}_{D}, \mathbf{v}_{D}\right)_{D}+\frac{\mathrm{F}}{\rho}\left(\left|\mathbf{u}_{D}\right| \mathbf{u}_{D}, \mathbf{v}_{D}\right)_{D},
\end{aligned}
$$


whereas the operator $\mathbf{b}: \mathbf{H} \rightarrow \mathbf{Q}^{\prime}$ is given by

$$
[\mathbf{b}(\mathbf{v}),(q, \xi)]:=-\left(\operatorname{div} \mathbf{v}_{S}, q\right)_{S}-\left(\operatorname{div} \mathbf{v}_{D}, q\right)_{D}+\left\langle\mathbf{v}_{S} \cdot \mathbf{n}-\mathbf{v}_{D} \cdot \mathbf{n}, \xi\right\rangle_{\Sigma} .
$$

In turn, the functionals $\mathbf{f}$ and $\mathbf{g}$ are defined by

$$
[\mathbf{f}, \mathbf{v}]:=\left(\mathbf{f}_{S}, \mathbf{v}_{S}\right)_{S}+\left(\mathbf{f}_{D}, \mathbf{v}_{D}\right)_{D} \quad \text { and } \quad[\mathbf{g},(q, \xi)]:=-\left(g_{D}, q\right)_{D} .
$$

In all the terms above, $[\cdot, \cdot]$ denotes the duality pairing induced by the corresponding operators. Further details for the solvability of (3.1) follows from the fixed-point strategy developed in Theorem 3.12 of [11].

\subsection{The finite element method}

Let $\mathcal{T}_{h}^{S}$ and $\mathcal{T}_{h}^{D}$ be respective triangulations of the domains $\Omega_{S}$ and $\Omega_{D}$ formed by shape-regular triangles of diameter $h_{T}$ and denote by $h_{S}$ and $h_{D}$ their corresponding mesh sizes. Assume that they match on $\Sigma$ so that $\mathcal{T}_{h}:=\mathcal{T}_{h}^{S} \cup \mathcal{T}_{h}^{D}$ is a triangulation of $\Omega:=\Omega_{S} \cup \Sigma \cup \Omega_{D}$. Hereafter $h:=\max \left\{h_{S}, h_{D}\right\}$. For each $T \in \mathcal{T}_{h}^{D}$ we consider the local Raviart-Thomas space of the lowest order:

$$
\mathbf{R T}_{0}(T):=\operatorname{span}\left\{(1,0),(0,1),\left(x_{1}, x_{2}\right)\right\} .
$$

In addition, for each $T \in \mathcal{T}_{h}^{S}$ we denote by $\mathbf{B R}(T)$ the local Bernardi-Raugel space:

$$
\mathbf{B R}(T):=\mathbf{P}_{1}(T) \oplus \operatorname{span}\left\{\eta_{2} \eta_{3} \mathbf{n}_{1}, \eta_{1} \eta_{3} \mathbf{n}_{2}, \eta_{1} \eta_{2} \mathbf{n}_{3}\right\},
$$

where $\left\{\eta_{1}, \eta_{2}, \eta_{3}\right\}$ are the barycentric coordinates of $T$, and $\left\{\mathbf{n}_{1}, \mathbf{n}_{2}, \mathbf{n}_{3}\right\}$ are the unit outward normals to the opposite sides of the corresponding vertices of $T$. Hence, the finite element subspaces for the velocities and pressure are, respectively,

$$
\begin{aligned}
\mathbf{H}_{h, \Gamma_{S}}\left(\Omega_{S}\right) & :=\left\{\mathbf{v} \in \mathbf{H}_{\Gamma_{S}}^{1}\left(\Omega_{S}\right):\left.\quad \mathbf{v}\right|_{T} \in \mathbf{B R}(T), \quad \forall T \in \mathcal{T}_{h}^{S}\right\}, \\
\mathbf{H}_{h, \Gamma_{D}}\left(\Omega_{D}\right) & :=\left\{\mathbf{v} \in \mathbf{H}_{\Gamma_{D}}^{3}\left(\operatorname{div} ; \Omega_{D}\right):\left.\quad \mathbf{v}\right|_{T} \in \mathbf{R T}_{0}(T), \quad \forall T \in \mathcal{T}_{h}^{D}\right\}, \\
L_{h, 0}(\Omega) & :=\left\{q \in L_{0}^{2}(\Omega):\left.\quad q\right|_{T} \in P_{0}(T), \quad \forall T \in \mathcal{T}_{h}\right\} .
\end{aligned}
$$

Next, for introducing the finite element subspace of $W^{1 / 3,3 / 2}(\Sigma)$, we denote by $\Sigma_{h}$ the partition of $\Sigma$ inherited from $\mathcal{T}_{h}^{D}\left(\right.$ or $\mathcal{T}_{h}^{S}$ ), which is formed by edges $e$ of length $h_{e}$, and set $h_{\Sigma}:=\max \left\{h_{e}: e \in \Sigma_{h}\right\}$. Therefore, we can define (see [11], Sect. 4 for details):

$$
\Lambda_{h}(\Sigma):=\left\{\xi_{h}: \Sigma \rightarrow \mathbb{R}:\left.\quad \xi_{h}\right|_{e} \in P_{0}(e) \quad \forall \text { edge } e \in \Sigma_{h}\right\} .
$$

In this way, grouping the discrete spaces and unknowns as follows:

$$
\begin{array}{ll}
\mathbf{H}_{h}:=\mathbf{H}_{h, \Gamma_{S}}\left(\Omega_{S}\right) \times \mathbf{H}_{h, \Gamma_{D}}\left(\Omega_{D}\right), & \mathbf{Q}_{h}:=L_{h, 0}(\Omega) \times \Lambda_{h}(\Sigma), \\
\mathbf{u}_{h}:=\left(\mathbf{u}_{S, h}, \mathbf{u}_{D, h}\right) \in \mathbf{H}_{h}, & \left(p_{h}, \lambda_{h}\right) \in \mathbf{Q}_{h},
\end{array}
$$

where $p_{h}:=p_{S, h} \chi_{S}+p_{D, h} \chi_{D}$, the Galerkin approximation of (3.1) reads: Find $\left(\mathbf{u}_{h},\left(p_{h}, \lambda_{h}\right)\right) \in \mathbf{H}_{h} \times \mathbf{Q}_{h}$, such that

$$
\begin{array}{ll}
{\left[\mathbf{a}_{h}\left(\mathbf{u}_{S, h}\right)\left(\mathbf{u}_{h}\right), \mathbf{v}_{h}\right]+\left[\mathbf{b}\left(\mathbf{v}_{h}\right),\left(p_{h}, \lambda_{h}\right)\right]=\left[\mathbf{f}, \mathbf{v}_{h}\right]} & \forall \mathbf{v}_{h}:=\left(\mathbf{v}_{S, h}, \mathbf{v}_{D, h}\right) \in \mathbf{H}_{h}, \\
{\left[\mathbf{b}\left(\mathbf{u}_{h}\right),\left(q_{h}, \xi_{h}\right)\right]=\left[\mathbf{g},\left(q_{h}, \xi_{h}\right)\right]} & \forall\left(q_{h}, \xi_{h}\right) \in \mathbf{Q}_{h} .
\end{array}
$$


Here, $\mathbf{a}_{h}\left(\mathbf{w}_{S, h}\right): \mathbf{H}_{h} \rightarrow \mathbf{H}_{h}^{\prime}$ is the discrete version of $\mathbf{a}\left(\mathbf{w}_{S}\right)\left(\right.$ with $\mathbf{w}_{S, h} \in \mathbf{H}_{h, \Gamma_{S}}\left(\Omega_{S}\right)$ in place of $\mathbf{w}_{S} \in \mathbf{H}_{\Gamma_{S}}^{1}\left(\Omega_{S}\right)$ ) defined by

$$
\left[\mathbf{a}_{h}\left(\mathbf{w}_{S, h}\right)\left(\mathbf{u}_{h}\right), \mathbf{v}_{h}\right]:=\left[\mathcal{A}_{S}\left(\mathbf{u}_{S, h}\right), \mathbf{v}_{S, h}\right]+\left[\mathcal{B}_{S}^{h}\left(\mathbf{w}_{S, h}\right)\left(\mathbf{u}_{S, h}\right), \mathbf{v}_{S, h}\right]+\left[\mathcal{A}_{D}\left(\mathbf{u}_{D, h}\right), \mathbf{v}_{D, h}\right],
$$

where $\mathcal{B}_{S}^{h}\left(\mathbf{w}_{S, h}\right)$ is the well-known skew-symmetric convective form:

$$
\left[\mathcal{B}_{S}^{h}\left(\mathbf{w}_{S, h}\right)\left(\mathbf{u}_{S, h}\right), \mathbf{v}_{S, h}\right]:=\rho\left(\left(\nabla \mathbf{u}_{S, h}\right) \mathbf{w}_{S, h}, \mathbf{v}_{S, h}\right)_{S}+\frac{\rho}{2}\left(\operatorname{div} \mathbf{w}_{S, h} \mathbf{u}_{S, h}, \mathbf{v}_{S, h}\right)_{S},
$$

for all $\mathbf{u}_{S, h}, \mathbf{v}_{S, h}, \mathbf{w}_{S, h} \in \mathbf{H}_{h, \Gamma_{S}}\left(\Omega_{S}\right)$. Moreover, we recall from equation (4.4) of [11] that for all $\mathbf{w}_{S, h}, \mathbf{u}_{S, h}, \mathbf{v}_{S, h} \in$ $\mathbf{H}_{h, \Gamma_{S}}\left(\Omega_{S}\right)$ there holds

$$
\left|\left[\mathcal{B}_{S}^{h}\left(\mathbf{w}_{S, h}\right)\left(\mathbf{u}_{S, h}\right), \mathbf{v}_{S, h}\right]\right| \leq C_{\mathrm{sk}}\left\|\mathbf{w}_{S, h}\right\|_{1, \Omega_{S}}\left\|\mathbf{u}_{S, h}\right\|_{1, \Omega_{S}}\left\|\mathbf{v}_{S, h}\right\|_{1, \Omega_{S}}
$$

with $C_{\mathrm{sk}}:=\rho C^{2}\left(\Omega_{S}\right)\left(1+\frac{\sqrt{2}}{2}\right)$, where $C\left(\Omega_{S}\right)$ is the norm of the injection of $H^{1}\left(\Omega_{S}\right)$ into $L^{4}\left(\Omega_{S}\right)(c f$. [11], Eq. (2.19)). The inequality (3.5) will be employed later on to derive the estimate (5.21) within the proof of Lemma 5.2, which is the starting point of the reliability analysis for the a posteriori error estimator to be proposed in Section 5 .

The solvability analysis and a priori error bounds for (3.3) are established in Theorems 4.9 and 5.2 of [11], respectively.

\section{Preliminaries for the a posteriori error analysis}

Now we introduce a few useful notations for describing local information on elements and edges. First, given $T \in \mathcal{T}_{h}^{S} \cup \mathcal{T}_{h}^{D}$, we let $\mathcal{E}(T)$ be the set of edges of $T$, and denote by $\mathcal{E}_{h}$ the set of all edges of $\mathcal{T}_{h}^{S} \cup \mathcal{T}_{h}^{D}$, subdivided as follows:

$$
\mathcal{E}_{h}=\mathcal{E}_{h}\left(\Gamma_{S}\right) \cup \mathcal{E}_{h}\left(\Gamma_{D}\right) \cup \mathcal{E}_{h}\left(\Omega_{S}\right) \cup \mathcal{E}_{h}\left(\Omega_{D}\right) \cup \mathcal{E}_{h}(\Sigma),
$$

where $\mathcal{E}_{h}\left(\Gamma_{\star}\right):=\left\{e \in \mathcal{E}_{h}: e \subseteq \Gamma_{\star}\right\}, \mathcal{E}_{h}\left(\Omega_{\star}\right):=\left\{e \in \mathcal{E}_{h}: e \subseteq \Omega_{\star}\right\}$, for $\star \in\{S, D\}$, and the edges of $\mathcal{E}_{h}(\Sigma)$ are exactly those forming the previously defined partition $\Sigma_{h}$, that is $\mathcal{E}_{h}(\Sigma):=\left\{e \in \mathcal{E}_{h}: e \subseteq \Sigma\right\}$. Moreover, $h_{e}$ stands for the length of a given edge $e$. Also for each edge $e \in \mathcal{E}_{h}$ we fix a unit normal vector $\mathbf{n}_{e}:=\left(n_{1}, n_{2}\right)^{t}$, and let $\mathbf{t}_{e}:=\left(-n_{2}, n_{1}\right)^{t}$ be the corresponding fixed unit tangential vector along $e$. Now, let $\mathbf{v} \in \mathbf{L}^{2}\left(\Omega_{\star}\right)$ such that $\left.\mathbf{v}\right|_{T} \in \mathbf{C}(T)$ on each $T \in \mathcal{T}_{h}^{\star}$. Then, given $e \in \mathcal{E}(T) \cap \mathcal{E}_{h}\left(\Omega_{\star}\right)$, we denote by $\llbracket \mathbf{v} \cdot \mathbf{t}_{e} \rrbracket$ the tangential jump of $\mathbf{v}$ across $e$, that is, $\llbracket \mathbf{v} \cdot \mathbf{t}_{e} \rrbracket:=\left(\left.\left(\left.\mathbf{v}\right|_{T}\right)\right|_{e}-\left.\left(\left.\mathbf{v}\right|_{T^{\prime}}\right)\right|_{e}\right) \cdot \mathbf{t}_{e}$, where $T$ and $T^{\prime}$ are the triangles of $\mathcal{T}_{h}^{\star}$ having $e$ as a common edge. In addition, for $\boldsymbol{\tau} \in \mathbb{L}^{2}\left(\Omega_{\star}\right)$ such that $\left.\boldsymbol{\tau}\right|_{T} \in \mathbb{C}(T)$, we let $\llbracket \boldsymbol{\tau} \mathbf{n}_{e} \rrbracket$ be the normal jump of $\boldsymbol{\tau}$ across $e$, that is, $\llbracket \boldsymbol{\tau} \mathbf{n}_{e} \rrbracket:=\left(\left.\left(\left.\boldsymbol{\tau}\right|_{T}\right)\right|_{e}-\left.\left(\left.\boldsymbol{\tau}\right|_{T^{\prime}}\right)\right|_{e}\right) \mathbf{n}_{e}$ and we let $\llbracket \boldsymbol{\tau} \mathbf{t}_{e} \rrbracket$ be the tangential jump of $\boldsymbol{\tau}$ across $e$, that is, $\llbracket \boldsymbol{\tau} \mathbf{t}_{e} \rrbracket:=\left(\left.\left(\left.\boldsymbol{\tau}\right|_{T}\right)\right|_{e}-\left.\left(\left.\boldsymbol{\tau}\right|_{T^{\prime}}\right)\right|_{e}\right) \mathbf{t}_{e}$. From now on, when no confusion arises, we simply write $\mathbf{n}$ and $\mathbf{t}$ instead of $\mathbf{n}_{e}$ and $\mathbf{t}_{e}$, respectively. Finally, given scalar and vector valued fields $\phi$ and $\mathbf{v}=\left(v_{1}, v_{2}\right)^{t}$, respectively, we set

$$
\operatorname{curl}(\phi):=\left(\frac{\partial \phi}{\partial x_{2}},-\frac{\partial \phi}{\partial x_{1}}\right)^{t} \text { and } \operatorname{rot}(\mathbf{v}):=\frac{\partial v_{2}}{\partial x_{1}}-\frac{\partial v_{1}}{\partial x_{2}},
$$

where the derivatives involved are taken in the distributional sense.

Let us now recall the main properties of the Raviart-Thomas interpolator (see [20,24]) and the Clément operator onto the space of continuous piecewise linear functions [12,31]. We begin with the former, denoted $\Pi_{h}: \mathbf{H}^{1}\left(\Omega_{D}\right) \rightarrow \mathbf{H}_{h, \Gamma_{D}}\left(\Omega_{D}\right)$, which is characterized by the identity

$$
\int_{e} \Pi_{h}(\mathbf{v}) \cdot \mathbf{n}=\int_{e} \mathbf{v} \cdot \mathbf{n} \quad \forall \text { edge } e \text { of } \mathcal{T}_{h}^{\star}
$$


Moreover, as a straightforward consequence of (4.1), there holds:

$$
\operatorname{div}\left(\Pi_{h}(\mathbf{v})\right)=\mathcal{P}_{h}^{D}(\operatorname{div} \mathbf{v}),
$$

where $\mathcal{P}_{h}^{D}$ is the $L^{2}\left(\Omega_{D}\right)$-orthogonal projector onto the piecewise constant functions on $\Omega_{D}$. The local approximation properties of $\Pi_{h}$ are established in the following lemma. For the corresponding proof we refer the reader to [20] (see also [5]).

Lemma 4.1. There exist constants $c_{1}, c_{2}>0$, independent of $h$, such that for all $\mathbf{v} \in \mathbf{H}^{1}\left(\Omega_{D}\right)$ there holds

$$
\left\|\mathbf{v}-\Pi_{h} \mathbf{v}\right\|_{0, T} \leq c_{1} h_{T}\|\mathbf{v}\|_{1, T} \quad \forall T \in \mathcal{T}_{h}^{D}
$$

and

$$
\left\|\mathbf{v} \cdot \mathbf{n}-\Pi_{h} \mathbf{v} \cdot \mathbf{n}\right\|_{0, e} \leq c_{2} h_{e}^{1 / 2}\|\mathbf{v}\|_{1, T_{e}} \quad \forall e \in \mathcal{E}_{h},
$$

where $T_{e}$ is a triangle of $\mathcal{T}_{h}^{D}$ containing the edge e on its boundary.

In turn, given $p \in(1,+\infty)$, we make use of the Clément interpolation operator $I_{h}^{\star}: W^{1, p}\left(\Omega_{\star}\right) \rightarrow X_{h}\left(\Omega_{\star}\right)$, with $\star \in\{S, D\}$, where

$$
X_{h}\left(\Omega_{\star}\right):=\left\{v \in \mathcal{C}\left(\bar{\Omega}_{\star}\right):\left.\quad v\right|_{T} \in P_{1}(T) \quad \forall T \in \mathcal{T}_{h}^{\star}\right\} .
$$

The local approximation properties of this operator are established in the following lemma (see [30], Lem. 3.1 for details):

Lemma 4.2. For each $\star \in\{S, D\}$ there exist constants $c_{3}, c_{4}>0$, independent of $h_{\star}$, such that for all $v \in$ $W^{1, p}\left(\Omega_{\star}\right)$ there hold

$$
\left\|v-I_{h}^{\star} v\right\|_{L^{p}(T)} \leq c_{3} h_{T}\|v\|_{1, p ; \Delta_{\star}(T)} \quad \forall T \in \mathcal{T}_{h}^{\star},
$$

and

where

$$
\left\|v-I_{h}^{\star} v\right\|_{L^{p}(e)} \leq c_{4} h_{e}^{1-1 / p}\|v\|_{1, p ; \Delta_{\star}(e)} \quad \forall e \in \mathcal{E}_{h},
$$

$$
\Delta_{\star}(T):=\cup\left\{T^{\prime} \in \mathcal{T}_{h}^{\star}: T^{\prime} \cap T \neq \emptyset\right\} \quad \text { and } \quad \Delta_{\star}(e):=\cup\left\{T^{\prime} \in \mathcal{T}_{h}^{\star}: T^{\prime} \cap e \neq \emptyset\right\} .
$$

In particular, for $p=2$ a vector version of $I_{h}^{S}$, say $\mathbf{I}_{h}^{S}: \mathbf{H}^{1}\left(\Omega_{S}\right) \rightarrow \mathbf{X}_{h}\left(\Omega_{S}\right)$, which is defined component-wise by $I_{h}^{S}$, will be needed as well.

For the forthcoming analysis we will also utilize a stable Helmholtz decomposition for $\mathbf{H}_{\Gamma_{D}}^{3}\left(\operatorname{div} ; \Omega_{D}\right)$. In this regard, and in order to analyze a more general result, given $p \in[2,+\infty)$ we will consider the Banach space $\mathbf{H}_{\Gamma_{D}}^{p}\left(\operatorname{div} ; \Omega_{D}\right)$ introduced in Section 3.1, and analogously to [3] we remark in advance that the decomposition for $\mathbf{H}_{\Gamma_{D}}^{p}\left(\operatorname{div} ; \Omega_{D}\right)$ will require the boundary $\Gamma_{D}$ to lie in a "convex part" of $\Omega_{D}$, which means that there exists a convex domain containing $\Omega_{D}$, and whose boundary contains $\Gamma_{D}$. We begin by introducing the following subspaces of $W^{1, p}\left(\Omega_{D}\right)$,

$$
W_{\Gamma_{D}}^{1, p}\left(\Omega_{D}\right):=\left\{\eta_{D} \in W^{1, p}\left(\Omega_{D}\right): \quad \eta_{D}=0 \quad \text { on } \quad \Gamma_{D}\right\}
$$

and establishing a suitable Helmholtz decomposition of our space $\mathbf{H}_{\Gamma_{D}}^{p}\left(\operatorname{div} ; \Omega_{D}\right)$.

Lemma 4.3. Assume that $\Omega_{D}$ is a simply connected domain and that $\Gamma_{D}$ is contained in the boundary of a convex part of $\Omega_{D}$, that is there exists a convex domain $\Xi$ such that $\bar{\Omega}_{D} \subseteq \Xi$ and $\Gamma_{D} \subseteq \partial \Xi$. Then, for each $\mathbf{v}_{D} \in \mathbf{H}_{\Gamma_{D}}^{p}\left(\operatorname{div} ; \Omega_{D}\right)$ with $p \in[2,+\infty)$, there exist $\mathbf{w}_{D} \in \mathbf{H}^{1}\left(\Omega_{D}\right)$ and $\beta_{D} \in W_{\Gamma_{D}}^{1, p}\left(\Omega_{D}\right)$ such that

$$
\mathbf{v}_{D}=\mathbf{w}_{D}+\operatorname{curl} \beta_{D} \text { in } \Omega_{D},
$$

and

$$
\left\|\mathbf{w}_{D}\right\|_{1, \Omega_{D}}+\left\|\beta_{D}\right\|_{1, p ; \Omega_{D}} \leq C_{\text {hel }}\left\|\mathbf{v}_{D}\right\|_{\mathbf{H}^{p}\left(\operatorname{div} ; \Omega_{D}\right)},
$$

where $C_{\mathrm{hel}}$ is a positive constant independent of all the foregoing variables. 
Proof. Since $\operatorname{div} \mathbf{v}_{D} \in L^{2}\left(\Omega_{D}\right)$ for each $\mathbf{v}_{D} \in \mathbf{H}_{\Gamma_{D}}^{p}$ (div; $\Omega_{D}$ ), the first part of the proof proceeds using similar arguments to Section 4.8.4 of [31] (see also [3], Lem. 3.9). In fact, given $\mathbf{v}_{D} \in \mathbf{H}_{\Gamma_{D}}^{p}\left(\operatorname{div} ; \Omega_{D}\right)$, we let $z \in H^{2}(\Xi)$ be the unique weak solution of the boundary value problem:

$$
\Delta z=\left\{\begin{aligned}
\operatorname{div} \mathbf{v}_{D} & \text { in } \Omega_{D} \\
-\frac{1}{\left|\Xi \backslash \bar{\Omega}_{D}\right|} \int_{\Omega_{D}} \operatorname{div} \mathbf{v}_{D} & \text { in } \Xi \backslash \bar{\Omega}_{D}, \quad \nabla z \cdot \mathbf{n}=0 \quad \text { on } \quad \partial \Xi, \quad \int_{\Xi} z=0 .
\end{aligned}\right.
$$

Thanks to the elliptic regularity result of (4.5) we have that $z \in H^{2}(\Xi)$ and

$$
\|z\|_{2, \Xi} \leq c\left\|\operatorname{div} \mathbf{v}_{D}\right\|_{0, \Omega_{D}},
$$

where $c>0$ is independent of $z$. In addition, it is clear that $\mathbf{w}_{D}:=\left.(\nabla z)\right|_{\Omega_{D}} \in \mathbf{H}^{1}\left(\Omega_{D}\right), \operatorname{div} \mathbf{w}_{D}=\Delta z=\operatorname{div} \mathbf{v}_{D}$ in $\Omega_{D}, \mathbf{w}_{D} \cdot \mathbf{n}=0$ on $\partial \Xi$ (which implies $\mathbf{w}_{D} \cdot \mathbf{n}=0$ on $\Gamma_{D}$ ), and

$$
\left\|\mathbf{w}_{D}\right\|_{1, \Omega_{D}} \leq\|z\|_{2, \Omega_{D}} \leq\|z\|_{2, \Xi} \leq c\left\|\operatorname{div} \mathbf{v}_{D}\right\|_{0, \Omega_{D}} .
$$

On the other hand, let us set $\phi_{D}:=\mathbf{v}_{D}-\mathbf{w}_{D}$ and notice that $\phi_{D}$ is a divergence-free vector field in $\Omega_{D}$. Then, using the continuous injection from $H^{1}\left(\Omega_{D}\right)$ into $L^{p}\left(\Omega_{D}\right)$ with $p \in[2,+\infty)$, and the estimate (4.6), we deduce that $\phi_{D} \in \mathbf{L}^{p}\left(\Omega_{D}\right)$ and

$$
\left\|\phi_{D}\right\|_{\mathbf{L}^{p}\left(\Omega_{D}\right)} \leq \widehat{c}\left\{\left\|\mathbf{v}_{D}\right\|_{\mathbf{L}^{p}\left(\Omega_{D}\right)}+\left\|\mathbf{w}_{D}\right\|_{1, \Omega_{D}}\right\} \leq \widetilde{c}\left\|\mathbf{v}_{D}\right\|_{\mathbf{H}^{p}\left(\operatorname{div} ; \Omega_{D}\right)}
$$

In this way, as a consequence of Theorem I.3.1 from [24], given $\phi_{D} \in \mathbf{L}^{p}\left(\Omega_{D}\right)$ with $p \in[2,+\infty)$ satisfying $\operatorname{div} \phi_{D}=0$ in $\Omega_{D}$, and $\Omega_{D}$ simply connected, there exists $\beta_{D} \in W^{1, p}\left(\Omega_{D}\right)$ such that $\phi_{D}=\operatorname{curl} \beta_{D}$ in $\Omega_{D}$, or equivalently

$$
\mathbf{v}_{D}-\mathbf{w}_{D}=\operatorname{curl} \beta_{D} \quad \text { in } \quad \Omega_{D} .
$$

In turn, noting that $0=\left(\mathbf{v}_{D}-\mathbf{w}_{D}\right) \cdot \mathbf{n}=\operatorname{curl} \beta_{D} \cdot \mathbf{n}=\frac{\mathrm{d} \beta_{D}}{\mathrm{~d} \mathbf{t}}$ on $\Gamma_{D}$, we deduce that $\beta_{D}$ is constant on $\Gamma_{D}$, and therefore $\beta_{D}$ can be chosen so that $\beta_{D} \in W_{\Gamma_{D}}^{1, p}\left(\Omega_{D}\right)$, which, together with (4.8), complete the Helmholtz decomposition (4.3). Finally, as a consequence of the generalized Poincaré inequality, it is easy to see that the norm $\left\|\beta_{D}\right\|_{1, p ; \Omega_{D}}$ and the seminorm $\left|\beta_{D}\right|_{1, p ; \Omega_{D}}=\left\|\operatorname{curl} \beta_{D}\right\|_{\mathbf{L}^{p}\left(\Omega_{D}\right)}$ are equivalent in $W_{\Gamma_{D}}^{1, p}\left(\Omega_{D}\right)$ (see [24], Lem. I.3.1 for details), so that employing (4.7), we obtain

$$
\left\|\beta_{D}\right\|_{1, p ; \Omega_{D}} \leq c\left\|\operatorname{curl} \beta_{D}\right\|_{\mathbf{L}^{p}\left(\Omega_{D}\right)}=c\left\|\phi_{D}\right\|_{\mathbf{L}^{p}\left(\Omega_{D}\right)} \leq C\left\|\mathbf{v}_{D}\right\|_{\mathbf{H}^{p}\left(\operatorname{div} ; \Omega_{D}\right)} .
$$

Then, it is clear that (4.6) and (4.9) imply (4.4) and conclude the proof.

Two very useful Green's formulae are recalled next.

Lemma 4.4. Let $p$ and $q$ be two fixed real numbers with $p \geq 1$ and $1 / p+1 / q=1$, and let $\Omega$ be a bounded domain with Lipschitz-continuous boundary $\partial \Omega$. Then there holds

$$
\int_{\Omega} \phi \operatorname{div} \mathbf{v}+\int_{\Omega} \mathbf{v} \cdot \nabla \phi=\langle\mathbf{v} \cdot \mathbf{n}, \phi\rangle_{\partial \Omega} \quad \forall \mathbf{v} \in \mathbf{H}^{p}\left(\operatorname{div}_{p} ; \Omega\right), \forall \phi \in W^{1, q}(\Omega),
$$

and

$$
\int_{\Omega} \phi \operatorname{rot} \mathbf{v}-\int_{\Omega} \mathbf{v} \cdot \operatorname{curl} \phi=\langle\mathbf{v} \cdot \mathbf{t}, \phi\rangle_{\partial \Omega} \quad \forall \mathbf{v} \in \mathbf{H}^{q}\left(\operatorname{rot}_{q} ; \Omega\right), \forall \phi \in W^{1, p}(\Omega),
$$

where

$$
\mathbf{H}^{p}\left(\operatorname{div}_{p} ; \Omega\right):=\left\{\mathbf{v} \in \mathbf{L}^{p}(\Omega): \quad \operatorname{div} \mathbf{v} \in L^{p}(\Omega)\right\}
$$

and

$$
\mathbf{H}^{q}\left(\operatorname{rot}_{q} ; \Omega\right):=\left\{\mathbf{v} \in \mathbf{L}^{q}(\Omega): \quad \operatorname{rot} \mathbf{v} \in L^{q}(\Omega)\right\} .
$$


Proof. We refer to Corollaries B.57 and B.58 of [16] for details (see also [24], Eq. (2.17) and Thm. I.2.11).

Finally, we end this section with a lemma providing estimates in terms of local quantities for the $W^{-1 / q, q}(\Sigma)$ norms of functions in particular subspaces of $L^{q}(\Sigma)$, with $1<p<2$ and $1 / p+1 / q=1$. More precisely, having in mind the definition of $\Lambda_{h}(\Sigma)$ (cf. (3.2)), which is subspace of $W^{1 / q, p}(\Sigma)$, we introduce the orthogonal-type space

$$
\Lambda_{h}^{\perp}(\Sigma):=\left\{\lambda \in W^{-1 / q, q}(\Sigma) \cap L^{q}(\Sigma): \quad\left\langle\lambda, \xi_{h}\right\rangle_{\Sigma}=0 \quad \forall \xi_{h} \in \Lambda_{h}(\Sigma)\right\} .
$$

Then, the announced result is stated as follows.

Lemma 4.5. Let $p$ and $q$ be two fixed real numbers with $1<p<2$ and $1 / p+1 / q=1$. Then, there exists $C>0$, independent of the meshsizes, such that

$$
\|\lambda\|_{-1 / q, q ; \Sigma} \leq C\left\{\sum_{e \in \mathcal{E}_{h}(\Sigma)} h_{e}\|\lambda\|_{L^{q}(e)}^{q}\right\}^{1 / q} \quad \forall \lambda \in \Lambda_{h}^{\perp}(\Sigma) .
$$

Proof. Given $\lambda \in \Lambda_{h}^{\perp}(\Sigma)$, we first observe that $\lambda \in W^{-1 / q, q}(\Sigma)$ and that

$$
\|\lambda\|_{-1 / q, q ; \Sigma}=\sup _{\substack{\xi \in W^{1 / q, p}(\Sigma) \\ \xi \neq 0}} \frac{\langle\lambda, \xi\rangle_{\Sigma}}{\|\xi\|_{1 / q, p ; \Sigma}} .
$$

Then, since $\mathcal{P}_{\Sigma}(\xi) \in \Lambda_{h}(\Sigma)$ with $\mathcal{P}_{\Sigma}$ being the $L^{2}(\Sigma)$-orthogonal projection onto $\Lambda_{h}(\Sigma)$, it follows from (4.14), (4.16), and Hölder's inequality, that

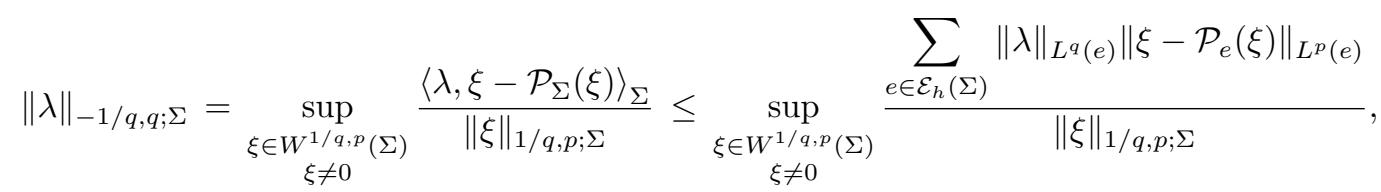

where $\mathcal{P}_{e}(\xi):=\left.\mathcal{P}_{\Sigma}(\xi)\right|_{e}$ on each $e \in \mathcal{E}_{h}(\Sigma)$. In turn, from the local approximation estimates of $\mathcal{P}_{e}$, we have

$$
\left\|\xi-\mathcal{P}_{e}(\xi)\right\|_{L^{p}(e)} \leq c h_{e}^{0}\|\xi\|_{L^{p}(e)} \quad \forall \xi \in L^{p}(e) \quad \text { and } \quad\left\|\xi-\mathcal{P}_{e}(\xi)\right\|_{L^{p}(e)} \leq c h_{e}\|\xi\|_{1, p ; e} \quad \forall \xi \in W^{1, p}(e),
$$

and then, by interpolation arguments, we find that

$$
\left\|\xi-\mathcal{P}_{e}(\xi)\right\|_{L^{p}(e)} \leq c h_{e}^{1 / q}\|\xi\|_{1 / q, p ; e} \quad \forall \xi \in W^{1 / q, p}(e),
$$

with $1 / p+1 / q=1$. Thus, the estimate (4.18) combined with (4.17), yields

$$
\begin{aligned}
& \sum_{e \in \mathcal{E}_{h}(\Sigma)}\|\lambda\|_{L^{q}(e)}\left\|\xi-\mathcal{P}_{e}(\xi)\right\|_{L^{p}(e)} \leq c \sum_{e \in \mathcal{E}_{h}(\Sigma)} h_{e}^{1 / q}\|\lambda\|_{L^{q}(e)}\|\xi\|_{1 / q, p ; e} \\
& \leq C\left\{\sum_{e \in \mathcal{E}_{h}(\Sigma)} h_{e}\|\lambda\|_{L^{q}(e)}^{q}\right\}^{1 / q}\left\{\sum_{e \in \mathcal{E}_{h}(\Sigma)}\|\xi\|_{1 / q, p ; e}^{p}\right\}^{1 / p} \leq C\left\{\sum_{e \in \mathcal{E}_{h}(\Sigma)} h_{e}\|\lambda\|_{L^{q}(e)}^{q}\right\}^{1 / q}\|\xi\|_{1 / q, p ; \Sigma} .
\end{aligned}
$$

Notice that in the last inequality we have used the fact that the space $\prod_{e \in \mathcal{E}_{h}(\Sigma)} W^{1 / q, p}(e)$ coincides with $W^{1 / q, p}(\Sigma)$, without extra conditions when $1<p<2$ ([25], Thm. 1.5.2.3-(a)), to obtain the norm $\|\xi\|_{1 / q, p ; \Sigma}$, which combined with (4.17) imply (4.15) and conclude the proof. 


\section{A POSTERIORI ERROR ANALYSIS}

Let $\overrightarrow{\mathbf{u}}:=(\mathbf{u},(p, \lambda)) \in \mathbf{H} \times \mathbf{Q}$ and $\overrightarrow{\mathbf{u}}_{h}:=\left(\mathbf{u}_{h},\left(p_{h}, \lambda_{h}\right)\right) \in \mathbf{H}_{h} \times \mathbf{Q}_{h}$ be the unique solutions of problems (3.1) and (3.3), respectively. In addition, let us denote $p_{S, h}:=\left.p_{h}\right|_{\Omega_{S}}$ and $p_{D, h}:=\left.p_{h}\right|_{\Omega_{D}}$. Then, we define for each $T \in \mathcal{T}_{h}^{S}$ the local error indicator

$$
\begin{aligned}
\Theta_{S, T}^{2}:= & \left\|\operatorname{div} \mathbf{u}_{S, h}\right\|_{0, T}^{2}+h_{T}^{2}\left\|\mathbf{f}_{S}+\operatorname{div} \boldsymbol{\sigma}_{S, h}-\rho\left(\nabla \mathbf{u}_{S, h}\right) \mathbf{u}_{S, h}-\frac{\rho}{2} \operatorname{div} \mathbf{u}_{S, h} \mathbf{u}_{S, h}\right\|_{0, T}^{2} \\
& +\sum_{e \in \mathcal{E}(T) \cap \mathcal{E}_{h}\left(\Omega_{S}\right)} h_{e}\left\|\llbracket \boldsymbol{\sigma}_{S, h} \mathbf{n} \rrbracket\right\|_{0, e}^{2}+\sum_{e \in \mathcal{E}(T) \cap \mathcal{E}_{h}(\Sigma)} h_{e}\left\|\boldsymbol{\sigma}_{S, h} \mathbf{n}+\lambda_{h} \mathbf{n}+\frac{\alpha_{d} \mu}{\sqrt{\mathbf{t} \cdot \boldsymbol{\kappa} \mathbf{t}}}\left(\mathbf{u}_{S, h} \cdot \mathbf{t}\right) \mathbf{t}\right\|_{0, e}^{2},
\end{aligned}
$$

where

$$
\boldsymbol{\sigma}_{S, h}:=-p_{S, h} \mathbb{I}+2 \mu \mathbf{e}\left(\mathbf{u}_{S, h}\right) \quad \text { on each } \quad T \in \mathcal{T}_{h}^{S} .
$$

Similarly, for each $T \in \mathcal{T}_{h}^{D}$ we set

$$
\widehat{\Theta}_{D, T}^{2}:=\left\|g_{D}-\operatorname{div} \mathbf{u}_{D, h}\right\|_{0, T}^{2}+h_{T}^{2}\left\|\mathbf{f}_{D}-\mathbf{U}_{D, h}\right\|_{0, T}^{2},
$$

and

$$
\widetilde{\Theta}_{D, T}^{3 / 2}:=\left\|\mathbf{f}_{D}-\mathbf{U}_{D, h}-\nabla \varphi_{h}\right\|_{\mathbf{L}^{3 / 2}(T)}^{3 / 2}
$$

where

$$
\mathbf{U}_{D, h}:=\frac{\mu}{\rho} \mathbf{K}^{-1} \mathbf{u}_{D, h}+\frac{\mathrm{F}}{\rho}\left|\mathbf{u}_{D, h}\right| \mathbf{u}_{D, h} \quad \text { on each } \quad T \in \mathcal{T}_{h}^{D},
$$

and $\varphi_{h}$ is any function in $W^{1,3 / 2}\left(\Omega_{D}\right)$ such that $\left.\varphi_{h}\right|_{\partial \Omega_{D}}=\widehat{\lambda}_{h}$, where $\widehat{\lambda}_{h}$ is the extension of $\lambda_{h}$ by the constant values of $p_{D}$ on the corresponding edges of $\Gamma_{D}$. Finally, for each $e \in \mathcal{E}_{h}(\Sigma)$ we define

$$
\Theta_{\Sigma, e}^{3}:=h_{e}\left\|\mathbf{u}_{S, h} \cdot \mathbf{n}-\mathbf{u}_{D, h} \cdot \mathbf{n}\right\|_{L^{3}(e)}^{3}
$$

so that the global a posteriori error estimator is given by:

$$
\Theta:=\left\{\sum_{T \in \mathcal{T}_{h}^{S}} \Theta_{S, T}^{2}+\sum_{T \in \mathcal{T}_{h}^{D}} \widehat{\Theta}_{D, T}^{2}\right\}^{1 / 2}+\left\{\sum_{T \in \mathcal{T}_{h}^{D}} \widetilde{\Theta}_{D, T}^{3 / 2}\right\}^{2 / 3}+\left\{\sum_{e \in \mathcal{E}_{h}(\Sigma)} \Theta_{\Sigma, e}^{3}\right\}^{1 / 3} .
$$

Notice that the second term of $\widehat{\Theta}_{D, T}^{2}$, and the full expression defining $\widetilde{\Theta}_{D, T}^{3 / 2}$, require that $\mathbf{f}_{D} \in \mathbf{L}^{2}(T)$ and $\mathbf{f}_{D} \in \mathbf{L}^{3 / 2}(T)$, respectively, for each $T \in \mathcal{T}_{h}^{D}$, which is ensured by assuming in what follows that $\mathbf{f}_{D}$ lives in $\mathbf{L}^{2}\left(\Omega_{D}\right)$. Then, the main goal of the present section is to establish, under suitable assumptions, the existence of positive constants $C_{\text {rel }}$ and $C_{\text {eff }}$, independent of the meshsizes and the continuous and discrete solutions, such that

$$
\begin{aligned}
\left\|\overrightarrow{\mathbf{u}}-\overrightarrow{\mathbf{u}}_{h}\right\|_{\mathbf{H} \times \mathbf{Q}} & \leq C_{\mathrm{rel}} \Theta^{1 / 2}, \text { and } \\
C_{\text {eff }} \Theta & \leq\left\|\overrightarrow{\mathbf{u}}-\overrightarrow{\mathbf{u}}_{h}\right\|_{\mathbf{H} \times \mathbf{Q}}+\left|p_{D}-\varphi_{h}\right|_{1,3 / 2 ; \Omega_{D}}+\text { h.o.t. }
\end{aligned}
$$

where h.o.t. stands, eventually, for one or several terms of higher order. The upper and lower bounds in (5.8), which are known as the reliability of $\Theta^{1 / 2}$ and efficiency of $\Theta$, are derived below in Sections 5.1 and 5.2, respectively. 


\subsection{Reliability}

First, we recall from [11] the following notation

$$
\mathcal{M}\left(\mathbf{f}_{S}, \mathbf{f}_{D}, g_{D}\right):=\max \left\{\mathcal{N}\left(\mathbf{f}_{S}, \mathbf{f}_{D}, g_{D}\right)^{1 / 2}, \mathcal{N}\left(\mathbf{f}_{S}, \mathbf{f}_{D}, g_{D}\right), \mathcal{N}\left(\mathbf{f}_{S}, \mathbf{f}_{D}, g_{D}\right)^{2}\right\}
$$

where $\mathcal{N}\left(\mathbf{f}_{S}, \mathbf{f}_{D}, g_{D}\right):=\left\|\mathbf{f}_{S}\right\|_{0, \Omega_{S}}+\left\|\mathbf{f}_{D}\right\|_{\mathbf{L}^{3 / 2}\left(\Omega_{D}\right)}+\left\|g_{D}\right\|_{0, \Omega_{D}}+\left\|g_{D}\right\|_{0, \Omega_{D}}^{2}$. Then, we establish the main result of this section.

Theorem 5.1. Assume that $\Omega_{D}$ is a simply connected domain and that $\Gamma_{D}$ is contained in the boundary of a convex part of $\Omega_{D}$, that is there exists a convex domain $\Xi$ such that $\bar{\Omega}_{D} \subseteq \Xi$ and $\Gamma_{D} \subseteq \partial \Xi$. In addition, assume that the data $\mathbf{f}_{S} \in \mathbf{L}^{2}\left(\Omega_{S}\right), \mathbf{f}_{D} \in \mathbf{L}^{2}\left(\Omega_{D}\right)$ and $g_{D} \in L^{2}\left(\Omega_{D}\right)$, satisfy:

$$
\mathcal{M}\left(\mathbf{f}_{S}, \mathbf{f}_{D}, g_{D}\right) \leq \frac{1}{2} \min \{r, \widetilde{r}\}
$$

where $r$ and $\widetilde{r}$ are the positive constants, independent of the data, provided by Lemma 3.11 and Theorem 4.9 of [11], respectively. Then, there exists a constant $C_{\mathrm{rel}}>0$, independent of $h$, such that

$$
\left\|\overrightarrow{\mathbf{u}}-\overrightarrow{\mathbf{u}}_{h}\right\|_{\mathbf{H} \times \mathbf{Q}} \leq C_{\mathrm{rel}} \Theta^{1 / 2} .
$$

We stress here that the data assumption (5.9) is required, on one hand, to ensure that the continuous and discrete problems, namely (3.1) and (3.3), are well-posed (see [11], Thms. 3.12 and 4.9 for details), and on the other hand, to prove next Lemma 5.2, which constitutes the first reliability estimate yielding (5.10). Later on, the same assumption is employed to establish the efficiency estimates given by (5.62), (5.63), and (5.64).

We begin the proof of (5.10) with a preliminary estimate for the total error $\left\|\overrightarrow{\mathbf{u}}-\overrightarrow{\mathbf{u}}_{h}\right\|_{\mathbf{H} \times \mathbf{Q}}$. In fact, proceeding analogously to Section 1 of [13] (see also [17,18]), we first define two residues $\mathcal{R}_{\mathbf{f}}$ and $\mathcal{R}_{\mathbf{g}}$ on $\mathbf{H}$ and $\mathbf{Q}$, respectively, by

$$
\mathcal{R}_{\mathbf{f}}(\mathbf{v}):=[\mathbf{f}, \mathbf{v}]-\left\{\left[\mathbf{a}_{h}\left(\mathbf{u}_{S, h}\right)\left(\mathbf{u}_{h}\right), \mathbf{v}\right]+\left[\mathbf{b}(\mathbf{v}),\left(p_{h}, \lambda_{h}\right)\right]\right\} \quad \forall \mathbf{v}:=\left(\mathbf{v}_{S}, \mathbf{v}_{D}\right) \in \mathbf{H}
$$

and

$$
\mathcal{R}_{\mathbf{g}}(q, \xi):=[\mathbf{g},(q, \xi)]-\left[\mathbf{b}\left(\mathbf{u}_{h}\right),(q, \xi)\right] \quad \forall(q, \xi) \in \mathbf{Q} .
$$

Then we are able to establish the following preliminary a posteriori error estimate.

Lemma 5.2. Assume that the data $\mathbf{f}_{S}, \mathbf{f}_{D}$ and $g_{D}$, satisfy (5.9). Then, there exists a constant $C>0$, depending only on parameters and other constants, all them independent of $h$, such that

$$
\left\|\overrightarrow{\mathbf{u}}-\overrightarrow{\mathbf{u}}_{h}\right\|_{\mathbf{H} \times \mathbf{Q}} \leq C \max \left\{\|\mathcal{R}\|_{(\mathbf{H} \times \mathbf{Q})^{\prime}}^{1 / 2},\|\mathcal{R}\|_{(\mathbf{H} \times \mathbf{Q})^{\prime}}^{2 / 3},\|\mathcal{R}\|_{(\mathbf{H} \times \mathbf{Q})^{\prime}}^{3 / 4},\|\mathcal{R}\|_{(\mathbf{H} \times \mathbf{Q})^{\prime}},\|\mathcal{R}\|_{(\mathbf{H} \times \mathbf{Q})^{\prime}}^{3 / 2}\right\},
$$

where $\mathcal{R}: \mathbf{H} \times \mathbf{Q} \rightarrow \mathbb{R}$ is the residual functional given by $\mathcal{R}(\overrightarrow{\mathbf{v}}):=\mathcal{R}_{\mathbf{f}}(\mathbf{v})+\mathcal{R}_{\mathbf{g}}(q, \xi) \quad \forall \overrightarrow{\mathbf{v}}:=(\mathbf{v},(q, \xi)) \in \mathbf{H} \times \mathbf{Q}$ (cf. (5.11) and (5.12)), which satisfies

$$
\mathcal{R}\left(\overrightarrow{\mathbf{v}}_{h}\right)=0 \quad \forall \overrightarrow{\mathbf{v}}_{h}:=\left(\mathbf{v}_{h},\left(q_{h}, \xi_{h}\right)\right) \in \mathbf{H}_{h} \times \mathbf{Q}_{h} .
$$

Proof. First, from the assumption (5.9) and the a priori estimates ([11] Thms. 3.12 and 4.9), we obtain

$$
\begin{aligned}
\max \left\{\left\|\mathbf{u}_{D}\right\|_{\mathbf{H}^{3}\left(\operatorname{div} ; \Omega_{D}\right)},\left\|\mathbf{u}_{S}\right\|_{1, \Omega_{S}}\right\} & \leq c_{\mathbf{T}} \mathcal{M}\left(\mathbf{f}_{S}, \mathbf{f}_{D}, g_{D}\right), \\
\max \left\{\left\|\mathbf{u}_{D, h}\right\|_{\mathbf{H}^{3}\left(\operatorname{div} ; \Omega_{D}\right)},\left\|\mathbf{u}_{S, h}\right\|_{1, \Omega_{S}}\right\} & \leq \widetilde{c}_{\mathbf{T}} \mathcal{M}\left(\mathbf{f}_{S}, \mathbf{f}_{D}, g_{D}\right) .
\end{aligned}
$$

In addition, since the exact solution $\mathbf{u}_{S} \in \mathbf{H}_{\Gamma_{S}}^{1}\left(\Omega_{S}\right)$ satisfies $\operatorname{div} \mathbf{u}_{S}=0$ in $\Omega_{S}$, we have

$$
\left[\mathcal{B}_{S}^{h}\left(\mathbf{u}_{S}\right)\left(\mathbf{u}_{S}\right), \mathbf{v}_{S, h}\right]=\left[\mathcal{B}_{S}\left(\mathbf{u}_{S}\right)\left(\mathbf{u}_{S}\right), \mathbf{v}_{S, h}\right] \quad \forall \mathbf{v}_{S, h} \in \mathbf{H}_{h, \Gamma_{S}}\left(\Omega_{S}\right)
$$


Consequently, from the continuous problem (3.1), and the definition of the residual functionals $\mathcal{R}_{\mathbf{f}}$ and $\mathcal{R}_{\mathbf{g}}(c f$. (5.11) and (5.12)), it is clear that

$$
\left[\mathbf{a}_{h}\left(\mathbf{u}_{S, h}\right)(\mathbf{u})-\mathbf{a}_{h}\left(\mathbf{u}_{S, h}\right)\left(\mathbf{u}_{h}\right), \mathbf{v}\right]+\left[\mathbf{b}(\mathbf{v}),\left(p-p_{h}, \lambda-\lambda_{h}\right)\right]=\mathcal{R}_{\mathbf{f}}(\mathbf{v})-\left[\mathcal{B}_{S}^{h}\left(\mathbf{u}_{S}-\mathbf{u}_{S, h}\right)\left(\mathbf{u}_{S}\right), \mathbf{v}_{S}\right],
$$

and

$$
\left[\mathbf{b}\left(\mathbf{u}-\mathbf{u}_{h}\right),(q, \xi)\right]=\mathcal{R}_{\mathbf{g}}(q, \xi)
$$

for all $\mathbf{v} \in \mathbf{H}$ and $(q, \xi) \in \mathbf{Q}$. Thus, from the inf-sup condition of $\mathbf{b}(c f$. [11], Lem. 3.5), the identity (5.16), and the continuity of $\mathbf{a}_{h}$ and $\mathcal{B}_{S}^{h}(c f$. [11], Lem. 4.3 and (3.5)), we deduce that

$$
\begin{aligned}
\beta\left\|\left(p-p_{h}, \lambda-\lambda_{h}\right)\right\|_{\mathbf{Q}} \leq & \sup _{\mathbf{0} \neq \mathbf{v} \in \mathbf{H}} \frac{\left[\mathbf{b}(\mathbf{v}),\left(p-p_{h}, \lambda-\lambda_{h}\right)\right]}{\|\mathbf{v}\|_{\mathbf{H}}} \\
\leq & \left\|\mathcal{R}_{\mathbf{f}}\right\|_{\mathbf{H}^{\prime}}+C_{1}\left(1+\left\|\mathbf{u}_{S}\right\|_{1, \Omega_{S}}+\left\|\mathbf{u}_{S, h}\right\|_{1, \Omega_{S}}\right)\left\|\mathbf{u}_{S}-\mathbf{u}_{S, h}\right\|_{1, \Omega_{S}} \\
& +C_{2}\left(1+\left\|\mathbf{u}_{D}\right\|_{\mathbf{H}^{3}\left(\operatorname{div} ; \Omega_{D}\right)}+\left\|\mathbf{u}_{D, h}\right\|_{\mathbf{H}^{3}\left(\operatorname{div} ; \Omega_{D}\right)}\right)\left\|\mathbf{u}_{D}-\mathbf{u}_{D, h}\right\|_{\mathbf{H}^{3}\left(\operatorname{div} ; \Omega_{D}\right)},
\end{aligned}
$$

which together with (5.15) and assumption (5.9), implies that there exists $C>0$, depending only on parameters and other constants, all of them independent of $h$, such that

$$
\left\|\left(p-p_{h}, \lambda-\lambda_{h}\right)\right\|_{\mathbf{Q}} \leq C\left\{\left\|\mathcal{R}_{\mathbf{f}}\right\|_{\mathbf{H}^{\prime}}+\left\|\mathbf{u}-\mathbf{u}_{h}\right\|_{\mathbf{H}}\right\} .
$$

In turn, taking $\mathbf{v}=\mathbf{u}-\mathbf{u}_{h}$ and $(q, \xi)=\left(p-p_{h}, \lambda-\lambda_{h}\right)$ in (5.16) and (5.17), respectively, gives

$$
\left[\mathbf{a}_{h}\left(\mathbf{u}_{S, h}\right)(\mathbf{u})-\mathbf{a}_{h}\left(\mathbf{u}_{S, h}\right)\left(\mathbf{u}_{h}\right), \mathbf{u}-\mathbf{u}_{h}\right]=\mathcal{R}_{\mathbf{f}}\left(\mathbf{u}-\mathbf{u}_{h}\right)-\mathcal{R}_{\mathbf{g}}\left(p-p_{h}, \lambda-\lambda_{h}\right)-\left[\mathcal{B}_{S}^{h}\left(\mathbf{u}_{S}-\mathbf{u}_{S, h}\right)\left(\mathbf{u}_{S}\right), \mathbf{u}_{S}-\mathbf{u}_{S, h}\right] .
$$

Next, employing the trace inequality, the estimate from the second row of (5.15), and the assumption (5.9), we get

$$
\left\|\mathbf{u}_{S, h} \cdot \mathbf{n}\right\|_{0, \Sigma} \leq C_{\mathrm{tr}}\left\|\mathbf{u}_{S, h}\right\|_{1, \Omega_{S}} \leq C_{\mathrm{tr}} \widetilde{c}_{\mathbf{T}} \mathcal{M}\left(\mathbf{f}_{S}, \mathbf{f}_{D}, g_{D}\right) \leq C_{\mathrm{tr}} \widetilde{c}_{\mathbf{T}} \frac{\widetilde{r}}{2}
$$

where $C_{\mathrm{tr}}$ is the norm of the usual trace operator from $H^{1}\left(\Omega_{S}\right)$ into $H^{1 / 2}\left(\partial \Omega_{S}\right)$. Then, using from Theorem 4.9 of [11] that

$$
\widetilde{r}=\frac{2 \mu \alpha_{S}}{\widetilde{c}_{\mathbf{T}} \rho} \min \left\{\frac{1}{C^{2}\left(\Omega_{S}\right)(2+\sqrt{2})}, \frac{1}{C^{2}\left(\partial \Omega_{S}\right) C_{\mathrm{tr}}^{3}}\right\},
$$

where $C\left(\partial \Omega_{S}\right)$ is the norm of the Sobolev embedding from $H^{1 / 2}\left(\partial \Omega_{S}\right)$ into $L^{4}\left(\partial \Omega_{S}\right)$, we deduce from (5.20) that

$$
\left\|\mathbf{u}_{S, h} \cdot \mathbf{n}\right\|_{0, \Sigma} \leq \frac{\mu \alpha_{S}}{\rho C^{2}\left(\partial \Omega_{S}\right) C_{\mathrm{tr}}^{2}} .
$$

This inequality shows that the assumption equation (4.15) of [11] is satisfied, and hence the operator $\mathbf{a}_{h}\left(\mathbf{u}_{S, h}\right)$ is strictly monotone with a constant $\alpha(\Omega)(c f$. [11], Eq. (3.15)), which, together with (5.19), yield

$$
\begin{aligned}
\alpha(\Omega)\left\{\left\|\mathbf{u}_{S}-\mathbf{u}_{S, h}\right\|_{1, \Omega_{S}}^{2}+\left\|\mathbf{u}_{D}-\mathbf{u}_{D, h}\right\|_{\mathbf{L}^{3}\left(\Omega_{D}\right)}^{3}\right\} \leq & \left\|\mathcal{R}_{\mathbf{f}}\right\|_{\mathbf{H}^{\prime}}\left\|\mathbf{u}-\mathbf{u}_{h}\right\|_{\mathbf{H}}+\left\|\mathcal{R}_{\mathbf{g}}\right\|_{\mathbf{Q}^{\prime}}\left\|\left(p-p_{h}, \lambda-\lambda_{h}\right)\right\|_{\mathbf{Q}} \\
& +C_{\text {sk }}\left\|\mathbf{u}_{S}\right\|_{1, \Omega_{S}}\left\|\mathbf{u}_{S}-\mathbf{u}_{S, h}\right\|_{1, \Omega_{S}}^{2},
\end{aligned}
$$

where in the last term of (5.21) we have used the continuity estimate of $\mathcal{B}_{S}^{h}(c f .(3.5))$. Moreover, using (5.15), (5.9), and the definition of $r$ in Lemma 3.11 of [11] to bound $C_{\mathrm{sk}}\left\|\mathbf{u}_{S}\right\|_{1, \Omega_{S}}$ by $\frac{2+\sqrt{2}}{4} \mu \alpha_{S}$, we deduce from (5.21) and (5.18), the existence of a constant $C>0$, independent of meshsizes, such that

$$
\left\|\mathbf{u}_{S}-\mathbf{u}_{S, h}\right\|_{1, \Omega_{S}}^{2}+\left\|\mathbf{u}_{D}-\mathbf{u}_{D, h}\right\|_{\mathbf{L}^{3}\left(\Omega_{D}\right)}^{3} \leq C\left\{\|\mathcal{R}\|_{(\mathbf{H} \times \mathbf{Q})^{\prime}}\left\|\mathbf{u}-\mathbf{u}_{h}\right\|_{\mathbf{H}}+\left\|\mathcal{R}_{\mathbf{f}}\right\|_{\mathbf{H}^{\prime}}\left\|\mathcal{R}_{\mathbf{g}}\right\|_{\mathbf{Q}^{\prime}}\right\} .
$$


Furthermore, from the identity (5.17) and the definition of $\mathbf{b}$ we find that the term $\left\|\operatorname{div}\left(\mathbf{u}_{D}-\mathbf{u}_{D, h}\right)\right\|_{0, \Omega_{D}}$ can be bounded by $\left\|\mathcal{R}_{\mathbf{g}}\right\|_{\mathbf{Q}^{\prime}}$, which, combined with (5.22) and some algebraic manipulations, implies

$$
\left\|\mathbf{u}-\mathbf{u}_{h}\right\|_{\mathbf{H}} \leq C \max \left\{\|\mathcal{R}\|_{(\mathbf{H} \times \mathbf{Q})^{\prime}}^{1 / 2},\|\mathcal{R}\|_{(\mathbf{H} \times \mathbf{Q})^{\prime}}^{2 / 3}\|\mathcal{R}\|_{(\mathbf{H} \times \mathbf{Q})^{\prime}}^{3 / 4},\|\mathcal{R}\|_{(\mathbf{H} \times \mathbf{Q})^{\prime}},\|\mathcal{R}\|_{(\mathbf{H} \times \mathbf{Q})^{\prime}}^{3 / 2}\right\} .
$$

Therefore, the estimate (5.13) follows from (5.18) and (5.23). Finally, from the discrete problem (3.3) we deduce that $\mathcal{R}_{\mathbf{f}}$ and $\mathcal{R}_{\mathbf{g}}$ vanish on $\mathbf{H}_{h}$ and $\mathbf{Q}_{h}$, respectively, which clearly implies (5.14) and conclude the proof.

We remark here that when $\|\mathcal{R}\|_{(\mathbf{H} \times \mathbf{Q})^{\prime}} \rightarrow 0$, the dominant term in (5.13) is $\|\mathcal{R}\|_{(\mathbf{H} \times \mathbf{Q})^{\prime}}^{1 / 2}$. In this way, it only remains now to estimate $\|\mathcal{R}\|_{(\mathbf{H} \times \mathbf{Q})^{\prime}}$. To this end, we first observe that the functional $\mathcal{R}$ can be decomposed as:

$$
\mathcal{R}(\overrightarrow{\mathbf{v}})=\mathcal{R}_{1}\left(\mathbf{v}_{S}\right)+\mathcal{R}_{2}\left(\mathbf{v}_{D}\right)+\mathcal{R}_{3}(q)+\mathcal{R}_{4}(\xi),
$$

for all $\overrightarrow{\mathbf{v}}:=\left(\left(\mathbf{v}_{S}, \mathbf{v}_{D}\right),(q, \xi)\right) \in \mathbf{H} \times \mathbf{Q}$, where

$$
\begin{aligned}
\mathcal{R}_{1}\left(\mathbf{v}_{S}\right):= & \left(\mathbf{f}_{S}, \mathbf{v}_{S}\right)_{S}-2 \mu\left(\mathbf{e}\left(\mathbf{u}_{S, h}\right), \mathbf{e}\left(\mathbf{v}_{S}\right)\right)_{S}-\rho\left(\left(\nabla \mathbf{u}_{S, h}\right) \mathbf{u}_{S, h}, \mathbf{v}_{S}\right)_{S}-\frac{\rho}{2}\left(\operatorname{div} \mathbf{u}_{S, h} \mathbf{u}_{S, h}, \mathbf{v}_{S}\right)_{S} \\
& +\left(\operatorname{div} \mathbf{v}_{S}, p_{h}\right)_{S}-\left\langle\frac{\alpha_{d} \mu}{\sqrt{\mathbf{t} \cdot \boldsymbol{\kappa} \mathbf{t}}} \mathbf{u}_{S, h} \cdot \mathbf{t}, \mathbf{v}_{S} \cdot \mathbf{t}\right\rangle_{\Sigma}-\left\langle\mathbf{v}_{S} \cdot \mathbf{n}, \lambda_{h}\right\rangle_{\Sigma}, \\
\mathcal{R}_{2}\left(\mathbf{v}_{D}\right):= & \left(\mathbf{f}_{D}-\mathbf{U}_{D, h}, \mathbf{v}_{D}\right)_{D}+\left(\operatorname{div} \mathbf{v}_{D}, p_{h}\right)_{D}+\left\langle\mathbf{v}_{D} \cdot \mathbf{n}, \lambda_{h}\right\rangle_{\Sigma}, \\
\mathcal{R}_{3}(q):= & \left(\operatorname{div} \mathbf{u}_{S, h}, q\right)_{S}-\left(g_{D}-\operatorname{div} \mathbf{u}_{D, h}, q\right)_{D}, \\
\mathcal{R}_{4}(\xi):= & -\left\langle\mathbf{u}_{S, h} \cdot \mathbf{n}-\mathbf{u}_{D, h} \cdot \mathbf{n}, \xi\right\rangle_{\Sigma} .
\end{aligned}
$$

Notice here that the above expression for $\mathcal{R}_{2}$ makes use of the definition of $\mathbf{U}_{D, h}$ given previously by (5.5). In this way, it follows that

$$
\|\mathcal{R}\|_{(\mathbf{H} \times \mathbf{Q})^{\prime}} \leq\left\{\left\|\mathcal{R}_{1}\right\|_{\mathbf{H}_{\Gamma_{S}}^{1}\left(\Omega_{S}\right)^{\prime}}+\left\|\mathcal{R}_{2}\right\|_{\mathbf{H}_{\Gamma_{D}}^{3}\left(\operatorname{div} ; \Omega_{D}\right)^{\prime}}+\left\|\mathcal{R}_{3}\right\|_{L_{0}^{2}(\Omega)^{\prime}}+\left\|\mathcal{R}_{4}\right\|_{W^{-1 / 3,3}(\Sigma)}\right\},
$$

and hence our next purpose is to derive suitable upper bounds for each one of the terms on the right-hand side of (5.26). We start with the following lemma, which is a direct consequence of the Cauchy-Schwarz inequality.

Lemma 5.3. There holds

$$
\left\|\mathcal{R}_{3}\right\|_{L_{0}^{2}(\Omega)^{\prime}} \leq\left\{\sum_{T \in \mathcal{T}_{h}^{S}}\left\|\operatorname{div} \mathbf{u}_{S, h}\right\|_{0, T}^{2}+\sum_{T \in \mathcal{T}_{h}^{D}}\left\|g_{D}-\operatorname{div} \mathbf{u}_{D, h}\right\|_{0, T}^{2}\right\}^{1 / 2} .
$$

We now adapt a result taken from [4] in order to obtain an upper bound for $\mathcal{R}_{1}$.

Lemma 5.4. There exists $C>0$, independent of the meshsizes, such that

$$
\left\|\mathcal{R}_{1}\right\|_{\mathbf{H}_{\Gamma_{S}}^{1}\left(\Omega_{S}\right)^{\prime}} \leq C\left\{\sum_{T \in \mathcal{T}_{h}^{S}} \widehat{\Theta}_{S, T}^{2}\right\}^{1 / 2},
$$

where

$$
\begin{aligned}
\widehat{\Theta}_{S, T}^{2}:= & h_{T}^{2}\left\|\mathbf{f}_{S}+\operatorname{div} \boldsymbol{\sigma}_{S, h}-\rho\left(\nabla \mathbf{u}_{S, h}\right) \mathbf{u}_{S, h}-\frac{\rho}{2} \operatorname{div} \mathbf{u}_{S, h} \mathbf{u}_{S, h}\right\|_{0, T}^{2} \\
& +\sum_{e \in \mathcal{E}(T) \cap \mathcal{E}_{h}\left(\Omega_{S}\right)} h_{e}\left\|\llbracket \boldsymbol{\sigma}_{S, h} \mathbf{n} \rrbracket\right\|_{0, e}^{2}+\sum_{e \in \mathcal{E}(T) \cap \mathcal{E}_{h}(\Sigma)} h_{e}\left\|\boldsymbol{\sigma}_{S, h} \mathbf{n}+\lambda_{h} \mathbf{n}+\frac{\alpha_{d} \mu}{\sqrt{\mathbf{t} \cdot \boldsymbol{\kappa} \mathbf{t}}}\left(\mathbf{u}_{S, h} \cdot \mathbf{t}\right) \mathbf{t}\right\|_{0, e}^{2},
\end{aligned}
$$

and $\boldsymbol{\sigma}_{S, h}$ is given by (5.2). 
Proof. We proceed similarly as in the proof of Lemma 3.4 from [4], by replacing $\mathbf{f}_{1}, \boldsymbol{\sigma}_{1, h}, \mathbf{u}_{1, h}, \Omega_{1}$, and $\Gamma_{2}$ in there by $\mathbf{f}_{S}-\rho\left(\nabla \mathbf{u}_{S, h}\right) \mathbf{u}_{S, h}-\frac{\rho}{2} \operatorname{div} \mathbf{u}_{S, h} \mathbf{u}_{S, h}, \boldsymbol{\sigma}_{S, h}, \mathbf{u}_{S, h}, \Omega_{S}$, and $\Sigma$, respectively, and employing the local approximation properties of the Clément interpolation operator $\mathbf{I}_{h}^{S}: \mathbf{H}^{1}\left(\Omega_{S}\right) \rightarrow \mathbf{X}_{h}\left(\Omega_{S}\right)$ provided by Lemma 4.2 with $p=2$. We omit further details.

Next, we derive the upper bound for $\mathcal{R}_{4}$, the functional acting on the interface $\Sigma$.

Lemma 5.5. There exists $C>0$, independent of the meshsizes, such that

$$
\left\|\mathcal{R}_{4}\right\|_{W^{-1 / 3,3}(\Sigma)} \leq C\left\{\sum_{e \in \mathcal{E}_{h}(\Sigma)} h_{e}\left\|\mathbf{u}_{S, h} \cdot \mathbf{n}-\mathbf{u}_{D, h} \cdot \mathbf{n}\right\|_{L^{3}(e)}^{3}\right\}^{1 / 3} .
$$

Proof. We recall from the definition of $\mathcal{R}_{4}(c f$. (5.25)) that

$$
\mathcal{R}_{4}(\xi)=-\left\langle\mathbf{u}_{S, h} \cdot \mathbf{n}-\mathbf{u}_{D, h} \cdot \mathbf{n}, \xi\right\rangle_{\Sigma} \quad \forall \xi \in W^{1 / 3,3 / 2}(\Sigma),
$$

which certainly yields

$$
\left\|\mathcal{R}_{4}\right\|_{W^{-1 / 3,3}(\Sigma)}=\left\|\mathbf{u}_{S, h} \cdot \mathbf{n}-\mathbf{u}_{D, h} \cdot \mathbf{n}\right\|_{-1 / 3,3 ; \Sigma} \cdot
$$

In turn, taking $\xi_{h} \in \Lambda_{h}(\Sigma)$ and then $\left(\mathbf{0},\left(0, \xi_{h}\right)\right) \in \mathbf{H}_{h} \times \mathbf{Q}_{h}$ in (5.14), we deduce that

$$
\left\langle\mathbf{u}_{S, h} \cdot \mathbf{n}-\mathbf{u}_{D, h} \cdot \mathbf{n}, \xi_{h}\right\rangle_{\Sigma}=0 \quad \forall \xi_{h} \in \Lambda_{h}(\Sigma),
$$

which says that $\mathbf{u}_{S, h} \cdot \mathbf{n}-\mathbf{u}_{D, h} \cdot \mathbf{n}$ belongs to $\Lambda_{h}^{\perp}(\Sigma)$ (cf. (4.14)). In this way, the proof follows from (5.27) and a direct application of (4.15) with $p=3 / 2$ and $q=3$ (cf. Lem. 4.5).

Finally, we focus on deriving the upper bound for $\mathcal{R}_{2}$, for which, given $\mathbf{v}_{D} \in \mathbf{H}_{\Gamma_{D}}^{3}$ (div; $\Omega_{D}$ ), we consider its Helmholtz decomposition provided by Lemma 4.3 with $p=3$. More precisely, we let $\mathbf{w}_{D} \in \mathbf{H}^{1}\left(\Omega_{D}\right)$ and $\beta_{D} \in W_{\Gamma_{D}}^{1,3}\left(\Omega_{D}\right)$ be such that $\mathbf{v}_{D}=\mathbf{w}_{D}+\operatorname{curl} \beta_{D}$ in $\Omega_{D}$, and

$$
\left\|\mathbf{w}_{D}\right\|_{1, \Omega_{D}}+\left\|\beta_{D}\right\|_{1,3 ; \Omega_{D}} \leq C_{\text {hel }}\left\|\mathbf{v}_{D}\right\|_{\mathbf{H}^{3}\left(\operatorname{div} ; \Omega_{D}\right)} .
$$

In turn, similarly to [3], we consider the finite element subspace of $W_{\Gamma_{D}}^{1,3}\left(\Omega_{D}\right)$ given by

$$
X_{h, \Gamma_{D}}:=\left\{v \in \mathcal{C}\left(\bar{\Omega}_{D}\right):\left.\quad v\right|_{T} \in P_{1}(T) \quad \forall T \in \mathcal{T}_{h}^{D}, \quad v=0 \quad \text { on } \quad \Gamma_{D}\right\}
$$

and introduce the Clément interpolator $I_{h}^{D}: W_{\Gamma_{D}}^{1,3}\left(\Omega_{D}\right) \rightarrow X_{h, \Gamma_{D}}$. In addition, recalling the Raviart-Thomas interpolator $\Pi_{h}: \mathbf{H}^{1}\left(\Omega_{D}\right) \rightarrow \mathbf{H}_{h, \Gamma_{D}}\left(\Omega_{D}\right)$ introduced in Section 4 , we are able to define

$$
\mathbf{v}_{D, h}:=\Pi_{h}\left(\mathbf{w}_{D}\right)+\operatorname{curl}\left(I_{h}^{D} \beta_{D}\right) \in \mathbf{H}_{h, \Gamma_{D}}\left(\Omega_{D}\right),
$$

which can be seen as a discrete Helmholtz decomposition of $\mathbf{v}_{D, h}$. Then, noting from (5.14) that $\mathcal{R}_{2}\left(\mathbf{v}_{D, h}\right)=0$, we can write

$$
\mathcal{R}_{2}\left(\mathbf{v}_{D}\right)=\mathcal{R}_{2}\left(\mathbf{v}_{D}-\mathbf{v}_{D, h}\right)=\mathcal{R}_{2}\left(\mathbf{w}_{D}-\Pi_{h}\left(\mathbf{w}_{D}\right)\right)+\mathcal{R}_{2}\left(\operatorname{curl}\left(\beta_{D}-I_{h}^{D} \beta_{D}\right)\right) .
$$

Next, in order to simplify the subsequent writing, we define $\widehat{\mathbf{w}}_{D}:=\mathbf{w}_{D}-\Pi_{h}\left(\mathbf{w}_{D}\right)$ and $\widehat{\beta}_{D}:=\beta_{D}-I_{h}^{D} \beta_{D}$. In this way, according to the definition of $\mathcal{R}_{2}(c f$. (5.25)), and employing the properties (4.1) and (4.2) of the Raviart-Thomas interpolator, which yield $\left\langle\widehat{\mathbf{w}}_{D} \cdot \mathbf{n}, \lambda_{h}\right\rangle_{\Sigma}=0$ and $\left(\operatorname{div} \widehat{\mathbf{w}}_{D}, p_{h}\right)_{D}=0$, respectively, we readily find that

$$
\mathcal{R}_{2}\left(\widehat{\mathbf{w}}_{D}\right)=\left(\mathbf{f}_{D}-\mathbf{U}_{D, h}, \widehat{\mathbf{w}}_{D}\right)_{D} .
$$

In turn, it follows straightforwardly from (5.25) as well that

$$
\mathcal{R}_{2}\left(\operatorname{curl} \widehat{\beta}_{D}\right)=\left(\mathbf{f}_{D}-\mathbf{U}_{D, h}, \operatorname{curl} \widehat{\beta}_{D}\right)_{D}+\left\langle\operatorname{curl} \widehat{\beta}_{D} \cdot \mathbf{n}, \lambda_{h}\right\rangle_{\Sigma} .
$$

The following lemma establishes the estimate for $\mathcal{R}_{2}$. 
Lemma 5.6. Assume that there exists a convex domain $\Xi$ such that $\Omega_{D} \subseteq \Xi$ and $\Gamma_{D} \subseteq \partial \Xi$. Assume further that $\mathbf{f}_{D} \in \mathbf{L}^{2}\left(\Omega_{D}\right)$. Then there exist $C_{1}, C_{2}>0$, independent of the meshsizes, such that

$$
\left\|\mathcal{R}_{2}\right\|_{\mathbf{H}^{3}\left(\operatorname{div} ; \Omega_{D}\right)^{\prime}} \leq C_{1}\left\{\sum_{T \in \mathcal{T}_{h}^{D}} h_{T}^{2}\left\|\mathbf{f}_{D}-\mathbf{U}_{D, h}\right\|_{0, T}^{2}\right\}^{1 / 2}+C_{2}\left\{\sum_{T \in \mathcal{T}_{h}^{D}} \widetilde{\Theta}_{D, T}^{3 / 2}\right\}^{2 / 3},
$$

where $\widetilde{\Theta}_{D, T}$ is defined in (5.4), and $\mathbf{U}_{D, h}$ is given by (5.5).

Proof. We begin by observing that the Cauchy-Schwarz inequality and the first approximation property of the Raviart-Thomas interpolation operator $\Pi_{h}$ in Lemma 4.1 imply

$$
\left|\mathcal{R}_{2}\left(\widehat{\mathbf{w}}_{D}\right)\right| \leq \widehat{C}_{1}\left\{\sum_{T \in \mathcal{T}_{h}^{D}} h_{T}^{2}\left\|\mathbf{f}_{D}-\mathbf{U}_{D, h}\right\|_{0, T}^{2}\right\}^{1 / 2}\left\|\mathbf{w}_{D}\right\|_{1, \Omega_{D}}
$$

We stress here that the term on the right-hand side of (5.33) is well defined thanks to the assumption $\mathbf{f}_{D} \in$ $\mathbf{L}^{2}\left(\Omega_{D}\right)$. On the other hand, in order to bound $\mathcal{R}_{2}\left(\operatorname{curl} \widehat{\beta}_{D}\right)(c f .(5.31))$, we begin by noticing that

$$
\left\langle\operatorname{curl} \widehat{\beta}_{D} \cdot \mathbf{n}, \lambda_{h}\right\rangle_{\Sigma}=\left\langle\operatorname{curl} \widehat{\beta}_{D} \cdot \mathbf{n}, E_{\Sigma}\left(\lambda_{h}\right)\right\rangle_{\partial \Omega_{D}}
$$

where $E_{\Sigma}: W^{1 / 3,3 / 2}(\Sigma) \rightarrow W^{1 / 3,3 / 2}\left(\partial \Omega_{D}\right)$ is any bounded extension operator. In particular, denoting by $\widehat{\lambda}_{h}$ the extension of $\lambda_{h}$ by the constant values of $p_{D}$ on the corresponding edges of $\Gamma_{D}$, and recalling from Theorem 1.5.2.3-(a) of [25] that, under no extra conditions, the product space $\prod_{e \in \mathcal{E}_{h}(\Sigma) \cup \mathcal{E}_{h}\left(\Gamma_{D}\right)} W^{1 / 3,3 / 2}(e)$ coincides with $W^{1 / 3,3 / 2}\left(\partial \Omega_{D}\right)$ through a linear isomorphism, we deduce that $\widehat{\lambda}_{h} \in W^{1 / 3,3 / 2}\left(\partial \Omega_{D}\right)$, so that we can write

$$
\left\langle\operatorname{curl} \widehat{\beta}_{D} \cdot \mathbf{n}, \lambda_{h}\right\rangle_{\Sigma}=\left\langle\operatorname{curl} \widehat{\beta}_{D} \cdot \mathbf{n}, \widehat{\lambda}_{h}\right\rangle_{\partial \Omega_{D}} .
$$

Now, in virtue of the surjectivity of the trace operator from $W^{1,3 / 2}\left(\Omega_{D}\right)$ onto $W^{1 / 3,3 / 2}\left(\partial \Omega_{D}\right)$, we know that there exists $\varphi_{h} \in W^{1,3 / 2}\left(\Omega_{D}\right)$ such that $\left.\varphi_{h}\right|_{\partial \Omega_{D}}=\widehat{\lambda}_{h}$. In this way, applying the Green formula (4.10) to $\mathbf{v}=\operatorname{curl} \widehat{\beta}_{D} \in \mathbf{H}^{3}\left(\operatorname{div}_{3} ; \Omega_{D}\right)$ and $\phi=\varphi_{h} \in W^{1,3 / 2}\left(\Omega_{D}\right)$, and recalling that $\mathbf{n}$ points towards inside $\Omega_{D}$ on $\Sigma$, we get

$$
\left\langle\operatorname{curl} \widehat{\beta}_{D} \cdot \mathbf{n}, \widehat{\lambda}_{h}\right\rangle_{\partial \Omega_{D}}=\left\langle\operatorname{curl} \widehat{\beta}_{D} \cdot \mathbf{n}, \varphi_{h}\right\rangle_{\partial \Omega_{D}}=-\int_{\Omega_{D}} \nabla \varphi_{h} \cdot \operatorname{curl} \widehat{\beta}_{D}
$$

which, in conjunction with (5.34) and (5.31), yields

$$
\mathcal{R}_{2}\left(\operatorname{curl} \widehat{\beta}_{D}\right)=\left(\mathbf{f}_{D}-\mathbf{U}_{D, h}-\nabla \varphi_{h}, \operatorname{curl} \widehat{\beta}_{D}\right)_{D} .
$$

Thus, applying Hölder's inequality in the right-hand side of (5.36), and then the boundedness of the Clément interpolator $I_{h}^{D}: W^{1,3}\left(\Omega_{D}\right) \rightarrow W^{1,3}\left(\Omega_{D}\right)(c f .[16]$, Lem. 1.127, p. 69), we obtain

$$
\left|\mathcal{R}_{2}\left(\operatorname{curl} \widehat{\beta}_{D}\right)\right| \leq \widehat{C}_{2}\left\{\sum_{T \in \mathcal{T}_{h}^{D}}\left\|\mathbf{f}_{D}-\mathbf{U}_{D, h}-\nabla \varphi_{h}\right\|_{\mathbf{L}^{3 / 2}(T)}^{3 / 2}\right\}^{2 / 3}\left\|\beta_{D}\right\|_{1,3 ; \Omega_{D}}
$$

Hence, as a direct consequence of estimates (5.33) and (5.37), and the stability estimate (5.28) for the Helmholtz decomposition, we get (5.32) and conclude the proof.

We end this section by stressing that the estimate (5.10) is a straightforward consequence of Lemmas 5.2-5.6, and the definition of the global estimator $\Theta(c f .(5.7))$, when $h \rightarrow 0$. 


\subsection{Efficiency}

The following theorem is the main result of this section.

Theorem 5.7. Suppose that the data $\mathbf{f}_{S} \in \mathbf{L}^{2}\left(\Omega_{S}\right), \mathbf{f}_{D} \in \mathbf{L}^{2}\left(\Omega_{D}\right)$ and $g_{D} \in L^{2}\left(\Omega_{D}\right)$ satisfy (5.9). Then, there exists a constant $C_{\text {eff }}>0$, independent of $h$, such that

$$
C_{\text {eff }} \Theta \leq\left\|\overrightarrow{\mathbf{u}}-\overrightarrow{\mathbf{u}}_{h}\right\|_{\mathbf{H} \times \mathbf{Q}}+\left|p_{D}-\varphi_{h}\right|_{1,3 / 2 ; \Omega_{D}}+\text { h.o.t., }
$$

where h.o.t. stands for one or several terms of higher order.

Throughout this section we assume, without loss of generality, that $\mathbf{K}^{-1} \mathbf{u}_{D, h}, \mathbf{f}_{S}, \mathbf{f}_{D}$, and $\varphi_{h}$ are all piecewise polynomials. Otherwise, if $\mathbf{K}, \mathbf{f}_{S}, \mathbf{f}_{D}$, and $\varphi_{h}$ are sufficiently smooth, and proceeding similarly as in Section 6.2 of [8], higher order terms given by the errors arising from suitable polynomial approximation of these expressions and functions would appear in (5.38), which explains the eventual h.o.t. in this inequality. More precisely, let us consider the $\mathbf{L}^{2}\left(\Omega_{\star}\right)$-orthogonal projector $\mathbf{P}_{k}^{\star}$ onto the vectorial piecewise polynomials of degree $\leq k$ on $\Omega_{\star}$, with $\star \in\{S, D\}$, which satisfies the following approximation property:

$\left(\mathbf{A P}_{k}^{\star}\right)$ there exists $C>0$, independent of $h$, such that for each $\mathbf{v} \in \mathbf{W}^{k+1, p}\left(\Omega_{\star}\right)$ there holds

$$
\left|\mathbf{v}-\mathbf{P}_{k}^{\star}(\mathbf{v})\right|_{k, p ; T} \leq C h_{T}|\mathbf{v}|_{k+1, p ; T} \quad \forall T \in \mathcal{T}_{h}^{\star} .
$$

Then, the h.o.t. arise when in the efficiency estimates to be derived below in Lemmas 5.12-5.15, the nonpolynomial data $\mathbf{f}_{S}$ and $\mathbf{f}_{D}$, and the given $\varphi_{h}$, are replaced by $\mathbf{P}_{k}^{S}\left(\mathbf{f}_{S}\right), \mathbf{P}_{k}^{D}\left(\mathbf{f}_{D}\right)$, and $\mathrm{P}_{k}^{D}\left(\varphi_{h}\right)$, respectively, with some $k \geq 0$, where $\mathrm{P}_{k}^{D}$ is the scalar version of $\mathbf{P}_{k}^{D}$. In particular, from the left hand side of (5.48) (cf. Lem. 5.12) we observe that, doing the above with $k=0$, the extra term $h_{T}^{2}\left\|\mathbf{f}_{S}-\mathbf{P}_{0}^{S}\left(\mathbf{f}_{S}\right)\right\|_{0, T}^{2}$ would appear, which can be bounded, using (5.39), as

$$
h_{T}^{2}\left\|\mathbf{f}_{S}-\mathbf{P}_{0}^{S}\left(\mathbf{f}_{S}\right)\right\|_{0, T}^{2} \leq C_{1} h_{T}^{4}\left|\mathbf{f}_{S}\right|_{1, T}^{2} \quad \forall T \in \mathcal{T}_{h}^{S},
$$

under the assumption that $\left.\mathbf{f}_{S}\right|_{T} \in \mathbf{H}^{1}(T)$ for all $T \in \mathcal{T}_{h}^{S}$. Proceeding similarly with the left hand side of (5.52) (cf. Lem. 5.15), we are lead to the extra term $h_{T}^{2}\left\|\mathbf{f}_{D}-\mathbf{P}_{0}^{D}\left(\mathbf{f}_{D}\right)\right\|_{0, T}^{2}$, and the estimate

$$
h_{T}^{2}\left\|\mathbf{f}_{D}-\mathbf{P}_{0}^{D}\left(\mathbf{f}_{D}\right)\right\|_{0, T}^{2} \leq C_{2} h_{T}^{4}\left|\mathbf{f}_{D}\right|_{1, T}^{2} \quad \forall T \in \mathcal{T}_{h}^{D},
$$

under the assumption that $\left.\mathbf{f}_{D}\right|_{T} \in \mathbf{H}^{1}(T)$ for all $T \in \mathcal{T}_{h}^{D}$. The respective terms in the other lemmas are handled analogously. Therefore, summing up over all $T \in \mathcal{T}_{h}^{\star}$, with $\star \in\{S, D\}$, in (5.40) and (5.41), the resulting expressions on the corresponding right hand sides become the h.o.t., which have a higher order of convergence than the rate of convergence $O\left(h^{1 / 3}\right)$ of the method (3.3) (see [11], Thm. 5.2 for details).

In order to prove (5.38) we need first to introduce the Banach space

$$
\mathbb{H}\left(\operatorname{div}_{4 / 3} ; \Omega_{S}\right):=\left\{\boldsymbol{\tau}_{S} \in \mathbb{L}^{2}\left(\Omega_{S}\right): \quad \operatorname{div} \boldsymbol{\tau}_{S} \in \mathbf{L}^{4 / 3}\left(\Omega_{S}\right)\right\}
$$

Then, we state the following result, which basically follows by applying integration by parts backwardly in the formulation (3.1), and proceeding as in Remark 2.1 of [6] for the Navier-Stokes terms.

Theorem 5.8. Let $(\mathbf{u},(p, \lambda)) \in \mathbf{H} \times \mathbf{Q}$ be the unique solution of (3.1). Then $\operatorname{div} \mathbf{u}_{S}=0$ in $\Omega_{S}$, $\operatorname{div} \mathbf{u}_{D}=g_{D}$ in $\Omega_{D}$, and $\mathbf{u}_{D} \cdot \mathbf{n}=\mathbf{u}_{S} \cdot \mathbf{n}$ on $\Sigma$. In addition, defining $p_{S}:=\left.p\right|_{\Omega_{S}}, p_{D}:=\left.p\right|_{\Omega_{D}}, \boldsymbol{\sigma}_{S}:=-p_{S} \mathbb{I}+2 \mu \mathbf{e}\left(\mathbf{u}_{S}\right)$, and $\mathbf{U}_{D}:=\frac{\mu}{\rho} \mathbf{K}^{-1} \mathbf{u}_{D}+\frac{\mathrm{F}}{\rho}\left|\mathbf{u}_{D}\right| \mathbf{u}_{D}$, there hold $p_{D} \in W^{1,3 / 2}\left(\Omega_{D}\right) \cap L^{2}\left(\Omega_{D}\right), \lambda=p_{D}$ on $\Sigma, \operatorname{div} \boldsymbol{\sigma}_{S}=\rho\left(\nabla \mathbf{u}_{S}\right) \mathbf{u}_{S}-\mathbf{f}_{S}$ in $\Omega_{S}$ (which yields $\boldsymbol{\sigma}_{S} \in \mathbb{H}\left(\mathbf{d i v}_{4 / 3} ; \Omega_{S}\right)$ ), $\mathbf{U}_{D}+\nabla p_{D}=\mathbf{f}_{D}$ in $\Omega_{D}$, and $\boldsymbol{\sigma}_{S} \mathbf{n}+\lambda \mathbf{n}+\frac{\alpha_{d} \mu}{\sqrt{\mathbf{t} \cdot \boldsymbol{\kappa} \mathbf{t}}}\left(\mathbf{u}_{S} \cdot \mathbf{t}\right) \mathbf{t}=\mathbf{0}$ on $\Sigma$.

We begin the derivation of the efficiency estimates with the following result.

Lemma 5.9. There hold

$$
\left\|\operatorname{div} \mathbf{u}_{S, h}\right\|_{0, T} \leq\left|\mathbf{u}_{S}-\mathbf{u}_{S, h}\right|_{1, T} \quad \forall T \in \mathcal{T}_{h}^{S}
$$

and

$$
\left\|g_{D}-\operatorname{div} \mathbf{u}_{D, h}\right\|_{0, T} \leq\left\|\mathbf{u}_{D}-\mathbf{u}_{D, h}\right\|_{\mathbf{H}^{3}(\operatorname{div} ; T)} \quad \forall T \in \mathcal{T}_{h}^{D} .
$$


Proof. It suffices to use from Theorem 5.8 that $\operatorname{div} \mathbf{u}_{S}=0$ in $\Omega_{S}$ and div $\mathbf{u}_{D}=g_{D}$ in $\Omega_{D}$. Further details are omitted.

In order to derive the upper bounds for the remaining terms defining the global a posteriori error estimator $\Theta$ (cf. (5.7)), we proceed similarly as in $[3,4,8,9,21]$, and apply results ultimately based on inverse inequalities and the localization technique based on triangle-bubble and edge-buble functions. To this end, we now recall some notation and introduce further preliminary results. Given $T \in \mathcal{T}_{h}:=\mathcal{T}_{h}^{S} \cup \mathcal{T}_{h}^{D}$, and $e \in \mathcal{E}(T)$, we let $\psi_{T}$ and $\psi_{e}$ be the usual triangle-bubble and edge-bubble functions, respectively (see [30], Eqs. (1.5) and (1.6)), which satisfy:

(i) $\psi_{T} \in P_{3}(T), \operatorname{supp}\left(\psi_{T}\right) \subseteq T, \psi_{T}=0$ on $\partial T$, and $0 \leq \psi_{T} \leq 1$ in $T$,

(ii) $\left.\psi_{e}\right|_{T} \in P_{2}(T), \operatorname{supp}\left(\psi_{e}\right) \subseteq \omega_{e}, \psi_{e}=0$ on $\partial T \backslash e$, and $0 \leq \psi_{e} \leq 1$ in $\omega_{e}:=\cup\left\{T^{\prime} \in \mathcal{T}_{h}: e \in \mathcal{E}\left(T^{\prime}\right)\right\}$.

In addition, we also recall from [30] that, given $k \in \mathbb{N} \cup\{0\}$, there exists an extension operator $L: \mathcal{C}(e) \rightarrow \mathcal{C}\left(\omega_{e}\right)$ that satisfies $L(\sigma) \in P_{k}(T)$ and $\left.L(\sigma)\right|_{e}=\sigma \forall \sigma \in P_{k}(e)$. A corresponding vectorial version of $L$, that is, the componentwise application of $L$, is denoted by $\mathbf{L}$. Additional properties of $\psi_{T}, \psi_{e}$, and $L$ are collected in the following lemma. Regarding the corresponding proof we refer to Lemma 3.3 of [30] for details.

Lemma 5.10. Let $p$ and $q$ be two fixed real numbers with $p \in[1,+\infty]$ and $1 / p+1 / q=1$. Given $T \in \mathcal{T}_{h}$ and $e \in \mathcal{E}(T)$, let $V_{T} \subset L^{\infty}(T)$ and $V_{e} \subset L^{\infty}(e)$ two arbitrary finite dimensional spaces. Then, there exist positive constants $c_{i}$ with $i \in\{1, \ldots, 7\}$, depending only on $p, q$, the spaces $V_{T}$ and $V_{e}$, and the shape-regularity of the triangulations (minimum angle condition), such that for each $u \in V_{T}$ and $\sigma \in V_{e}$, there hold

$$
\begin{aligned}
c_{1}\|u\|_{L^{p}(T)} & \leq \sup _{v \in V_{T}} \frac{\int_{T} u \psi_{T} v}{\|v\|_{L^{q}(T)}} \leq\|u\|_{L^{p}(T)}, \\
c_{2}\|\sigma\|_{L^{p}(e)} & \leq \sup _{\tau \in V_{e}} \frac{\int_{e} \sigma \psi_{e} \tau}{\|\tau\|_{L^{q}(e)}} \leq\|\sigma\|_{L^{p}(e)}, \\
c_{3} h_{T}^{-1}\left\|\psi_{T} u\right\|_{L^{q}(T)} & \leq\left\|\nabla\left(\psi_{T} u\right)\right\|_{L^{q}(T)} \leq c_{4} h_{T}^{-1}\left\|\psi_{T} u\right\|_{L^{q}(T)}, \\
c_{5} h_{T}^{-1}\left\|\psi_{e} L(\sigma)\right\|_{L^{q}(T)} & \leq\left\|\nabla\left(\psi_{e} L(\sigma)\right)\right\|_{L^{q}(T)} \leq c_{6} h_{T}^{-1}\left\|\psi_{e} L(\sigma)\right\|_{L^{q}(T)},
\end{aligned}
$$

and

$$
\left\|\psi_{e} L(\sigma)\right\|_{L^{q}(T)} \leq c_{7} h_{e}^{1 / q}\|\sigma\|_{L^{q}(e)} .
$$

As stated in Remark 3.2 of [30], $V_{T}$ and $V_{e}$ can be chosen as suitable spaces of polynomials. Thus, in what follows we will choose $V_{T}$ as $P_{k}(T)$ and $V_{e}$ as $P_{k}(e)$ for a given $k \in \mathbb{N} \cup\{0\}$. In addition, and coherently with previous notations, we set $\mathbf{V}_{T}$ and $\mathbf{V}_{e}$, respectively, as the corresponding vectorial counterpart. The following inverse estimate will be also used. We refer the reader to Lemma 1.138 of [16] for its proof.

Lemma 5.11. Let $k \in \mathbb{N} \cup\{0\}, n \in\{2,3\}, l, m \geq 0$ such that $m \leq l$, and $p, q \in[1,+\infty]$. Then, there exists $c>0$, depending only on $k, l, m$ and the shape regularity of the triangulations, such that, for each triangle (tetrahedron) $T \in \mathcal{T}_{h}$, there holds

$$
\|v\|_{l, p, T} \leq c h_{T}^{m-l+n(1 / p-1 / q)}\|v\|_{m, q, T} \quad \forall v \in P_{k}(T) .
$$

We point out that throughout this section each proof done in $2 \mathrm{D}$ can be easily extended to its threedimensional counterpart considering $n=3$ when we apply (5.47). In that case, other positive power of the meshsizes $h_{T_{\star}}$, with $\star \in\{S, D\}$, will appear on the right-hand side of the efficiency estimates which anyway are bounded. Next, we continue providing the corresponding efficiency estimates of our analysis with the upper bounds for the remaining three terms defining $\Theta_{S, T}^{2}(c f$. (5.1)). Since the corresponding proofs are adaptations 
to our configuration of those of Lemmas 4.4-4.6 from [4], we only mention the main tools employed and refer to the preprint version of the present paper ( $c f$. [10]) for details. However, for the sake of clearness and completeness, and because of some technicalities involved, we do provide full proofs for the estimates that include terms arising from the Darcy-Forchheimer equation.

Lemma 5.12. There exists $c>0$, independent of $h$, such that for each $T \in \mathcal{T}_{h}^{S}$ there holds

$$
\begin{gathered}
h_{T}^{2}\left\|\mathbf{f}_{S}+\operatorname{div} \boldsymbol{\sigma}_{S, h}-\rho\left(\nabla \mathbf{u}_{S, h}\right) \mathbf{u}_{S, h}-\frac{\rho}{2} \operatorname{div} \mathbf{u}_{S, h} \mathbf{u}_{S, h}\right\|_{0, T}^{2} \leq c\left\{\left\|p_{S}-p_{S, h}\right\|_{0, T}^{2}+\left|\mathbf{u}_{S}-\mathbf{u}_{S, h}\right|_{1, T}^{2}\right. \\
\left.+h_{T}\left\|\left(\nabla \mathbf{u}_{S}\right) \mathbf{u}_{S}-\left(\nabla \mathbf{u}_{S, h}\right) \mathbf{u}_{S, h}\right\|_{\mathbf{L}^{4 / 3}(T)}^{2}+h_{T}\left\|\operatorname{div}\left(\mathbf{u}_{S}-\mathbf{u}_{S, h}\right) \mathbf{u}_{S, h}\right\|_{\mathbf{L}^{4 / 3}(T)}^{2}\right\} .
\end{gathered}
$$

Proof. Given $T \in \mathcal{T}_{h}^{S}$, it proceeds by applying first (5.42) to $\left\|\boldsymbol{\chi}_{T}\right\|_{0, T}$, with $\boldsymbol{\chi}_{T}:=\mathbf{f}_{S}+\operatorname{div} \boldsymbol{\sigma}_{S, h}-\rho\left(\nabla \mathbf{u}_{S, h}\right) \mathbf{u}_{S, h}-$ $\frac{\rho}{2} \operatorname{div} \mathbf{u}_{S, h} \mathbf{u}_{S, h}$, and then employing Cauchy-Schwarz and Hölder's inequalities, (5.44), and the local inverse estimate (5.47). For further details we refer to Lemma 5.12 of [10].

Lemma 5.13. There exists $c>0$, independent of $h$, such that for each $e \in \mathcal{E}_{h}\left(\Omega_{S}\right)$ there holds

$$
\begin{aligned}
h_{e}\left\|\llbracket \boldsymbol{\sigma}_{S, h} \mathbf{n} \rrbracket\right\|_{0, e}^{2} \leq & c \sum_{T \subseteq \omega_{e}}\left\{\left\|p_{S}-p_{S, h}\right\|_{0, T}^{2}+\left|\mathbf{u}_{S}-\mathbf{u}_{S, h}\right|_{1, T}^{2}\right. \\
& \left.+h_{T}\left\|\left(\nabla \mathbf{u}_{S}\right) \mathbf{u}_{S}-\left(\nabla \mathbf{u}_{S, h}\right) \mathbf{u}_{S, h}\right\|_{\mathbf{L}^{4 / 3}(T)}^{2}+h_{T}\left\|\operatorname{div}\left(\mathbf{u}_{S}-\mathbf{u}_{S, h}\right) \mathbf{u}_{S, h}\right\|_{\mathbf{L}^{4 / 3}(T)}^{2}\right\},
\end{aligned}
$$

where $\omega_{e}$ is the union of the two triangles in $\mathcal{T}_{h}^{S}$ having e as an edge.

Proof. Given $e \in \mathcal{E}_{h}\left(\Omega_{S}\right)$, it begins by applying (5.43) to $\left\|\llbracket \boldsymbol{\sigma}_{S, h} \mathbf{n} \rrbracket\right\|_{0, e}$. The rest of the proof makes use of the Cauchy-Schwarz and Hölder inequalities, and the estimates (5.45)-(5.47). We refer to Lemma 5.13 of [10] for further details.

Before establishing the following lemma, we need to recall a local trace inequality ([1], Thm. 3.10). Indeed, there exists $c>0$, depending only on the shape regularity of the triangulations, such that for each $T \in \mathcal{T}_{h}^{S} \cup \mathcal{T}_{h}^{D}$ and $e \in \mathcal{E}(T)$, there holds

$$
h_{e}\|v\|_{0, e}^{2} \leq c\left\{\|v\|_{0, T}^{2}+h_{T}^{2}|v|_{1, T}^{2}\right\} \quad \forall v \in H^{1}(T) .
$$

Lemma 5.14. There exists $c>0$, independent of $h$, such that for each $e \in \mathcal{E}_{h}(\Sigma)$ there holds

$$
\begin{aligned}
h_{e}\left\|\boldsymbol{\sigma}_{S, h} \mathbf{n}+\lambda_{h} \mathbf{n}+\frac{\alpha_{d} \mu}{\sqrt{\mathbf{t} \cdot \boldsymbol{\kappa} \mathbf{t}}}\left(\mathbf{u}_{S, h} \cdot \mathbf{t}\right) \mathbf{t}\right\|_{0, e}^{2} \leq & c\left\{\left\|p_{S}-p_{S, h}\right\|_{0, T}^{2}+\left\|\mathbf{u}_{S}-\mathbf{u}_{S, h}\right\|_{1, T}^{2}+h_{e}\left\|\lambda-\lambda_{h}\right\|_{0, e}^{2}\right. \\
& +h_{T}\left\|\left(\nabla \mathbf{u}_{S}\right) \mathbf{u}_{S}-\left(\nabla \mathbf{u}_{S, h}\right) \mathbf{u}_{S, h}\right\|_{\mathbf{L}^{4 / 3}(T)}^{2} \\
& \left.+h_{T}\left\|\operatorname{div}\left(\mathbf{u}_{S}-\mathbf{u}_{S, h}\right) \mathbf{u}_{S, h}\right\|_{\mathbf{L}^{4 / 3}(T)}^{2}\right\},
\end{aligned}
$$

where $T$ is the triangle of $\mathcal{T}_{h}^{S}$ having e as an edge.

Proof. Given $e \in \mathcal{E}_{h}(\Sigma)$, we first apply (5.43) to $\left\|\chi_{e}\right\|_{0, e}$, with $\chi_{e}:=\boldsymbol{\sigma}_{S, h} \mathbf{n}+\lambda_{h} \mathbf{n}+\frac{\alpha_{d} \mu}{\sqrt{\mathbf{t} \cdot \boldsymbol{\kappa} \mathbf{t}}}\left(\mathbf{u}_{S, h} \cdot \mathbf{t}\right) \mathbf{t}$. Then, the proof continues by applying again the Cauchy-Schwarz and Hölder inequalities, and by using the estimates (5.45)-(5.47), and (5.50). We refer to Lemma 5.14 of [10] for all the details.

The second residual expression defining $\widehat{\Theta}_{D, T}^{2}(c f .(5.3))$, that is, the one containing the nonlinear DarcyForchheimer term, is estimated now. To that end, we adapt the proof of Lemma 6.3 from [7] to our context. 
Lemma 5.15. There exists $c>0$, independent of $h$, such that for each $T \in \mathcal{T}_{h}^{D}$ there holds

$$
h_{T}^{2}\left\|\mathbf{f}_{D}-\mathbf{U}_{D, h}\right\|_{0, T}^{2} \leq c\left\{\left\|p_{D}-p_{D, h}\right\|_{0, T}^{2}+h_{T}^{2}\left\|\mathbf{u}_{D}-\mathbf{u}_{D, h}\right\|_{\mathbf{L}^{3}(T)}^{2}+h_{T}^{4 / 3}\left\|\left|\mathbf{u}_{D}\right| \mathbf{u}_{D}-\left|\mathbf{u}_{D, h}\right| \mathbf{u}_{D, h}\right\|_{\mathbf{L}^{3 / 2}(T)}^{2}\right\} .
$$

Proof. Given $T \in \mathcal{T}_{h}^{D}$, we apply (5.42) to $\left\|\mathbf{f}_{D}-\mathbf{U}_{D, h}\right\|_{0, T}$, that is

$$
c_{1}\left\|\mathbf{f}_{D}-\mathbf{U}_{D, h}\right\|_{0, T} \leq \sup _{\mathbf{v} \in \mathbf{V}_{T}} \frac{\int_{T}\left(\mathbf{f}_{D}-\mathbf{U}_{D, h}\right) \cdot \psi_{T} \mathbf{v}}{\|\mathbf{v}\|_{0, T}},
$$

from which, using the identity $\mathbf{f}_{D}=\mathbf{U}_{D}+\nabla p_{D}$ in $\Omega_{D}$ (cf. Thm. 5.8), noting that $\nabla p_{D, h}=0$ on $T$, and integrating by parts, we find that

$$
\begin{aligned}
\int_{T}\left(\mathbf{f}_{D}-\mathbf{U}_{D, h}\right) \cdot \psi_{T} \mathbf{v} & =\int_{T}\left(\nabla\left(p_{D}-p_{D, h}\right)+\left(\mathbf{U}_{D}-\mathbf{U}_{D, h}\right)\right) \cdot \psi_{T} \mathbf{v} \\
& =-\int_{T}\left(p_{D}-p_{D, h}\right) \operatorname{div}\left(\psi_{T} \mathbf{v}\right)+\int_{T}\left(\mathbf{U}_{D}-\mathbf{U}_{D, h}\right) \cdot \psi_{T} \mathbf{v} .
\end{aligned}
$$

In this way, from the definitions of $\mathbf{U}_{D}$ and $\mathbf{U}_{D, h}$ ( $c f$. Thm. 5.8 and (5.5)), using the Cauchy-Schwarz and Hölder inequalities, applying (5.44) to $\left\|\nabla\left(\psi_{T} \mathbf{v}\right)\right\|_{0, T}$, and recalling that $0 \leq \psi_{T} \leq 1$, we get

$$
\begin{aligned}
& \int_{T}\left(\mathbf{f}_{D}-\mathbf{U}_{D, h}\right) \cdot \psi_{T} \mathbf{v} \leq C\left\{h_{T}^{-1}\left\|p_{D}-p_{D, h}\right\|_{0, T}+\left\|\mathbf{u}_{D}-\mathbf{u}_{D, h}\right\|_{0, T}\right\}\|\mathbf{v}\|_{0, T} \\
&+\left\|\left|\mathbf{u}_{D}\right| \mathbf{u}_{D}-\left|\mathbf{u}_{D, h}\right| \mathbf{u}_{D, h}\right\|_{\mathbf{L}^{3 / 2}(T)}\|\mathbf{v}\|_{\mathbf{L}^{3}(T)}
\end{aligned}
$$

Then, replacing (5.54) back into (5.53), and then applying Hölder's inequality and the local inverse estimate $\|\mathbf{v}\|_{\mathbf{L}^{3}(T)} \leq c h_{T}^{-1 / 3}\|\mathbf{v}\|_{0, T}(c f .(5.47))$, we arrive at (5.52) and complete the proof.

Now we turn to provide the upper bound for the term defining $\widetilde{\Theta}_{D, T}^{3 / 2}(c f .(5.4))$.

Lemma 5.16. There exists $c>0$, independent of $h$, such that for each $T \in \mathcal{T}_{h}^{D}$ there holds

$$
\left\|\mathbf{f}_{D}-\mathbf{U}_{D, h}-\nabla \varphi_{h}\right\|_{\mathbf{L}^{3 / 2}(T)}^{3 / 2} \leq c\left\{\left\|\mathbf{u}_{D}-\mathbf{u}_{D, h}\right\|_{\mathbf{L}^{3}(T)}^{3 / 2}+\left|p_{D}-\varphi_{h}\right|_{1,3 / 2 ; T}^{3 / 2}+\left\|\left|\mathbf{u}_{D}\right| \mathbf{u}_{D}-\left|\mathbf{u}_{D, h}\right| \mathbf{u}_{D, h}\right\|_{\mathbf{L}^{3 / 2}(T)}^{3 / 2}\right\} .
$$

Proof. We first recall from Theorem 5.8 that $\mathbf{f}_{D}=\mathbf{U}_{D}+\nabla p_{D}$ in $\Omega_{D}$. Then, replacing this identity in the expression $\left\|\mathbf{f}_{D}-\mathbf{U}_{D, h}-\nabla \varphi_{h}\right\|_{\mathbf{L}^{3 / 2}(T)}$, employing the definitions of $\mathbf{U}_{D}$ and $\mathbf{U}_{D, h}$ (cf. Thm. 5.8 and (5.5)), and applying the triangle and Hölder inequalities, we readily arrive at the estimate (5.55). Further details are omitted.

Finally, we provide the upper bound for the term defining $\Theta_{\Sigma, e}^{3}(c f .(5.6))$.

Lemma 5.17. There exists $c>0$, independent of $h$, such that for each $e \in \mathcal{E}_{h}(\Sigma)$ there holds

$$
\begin{aligned}
h_{e}\left\|\mathbf{u}_{S, h} \cdot \mathbf{n}-\mathbf{u}_{D, h} \cdot \mathbf{n}\right\|_{L^{3}(e)}^{3} \leq & c\left\{\left\|\mathbf{u}_{S}-\mathbf{u}_{S, h}\right\|_{\mathbf{L}^{3}\left(T_{S}\right)}^{3}+h_{T_{S}}^{2}\left|\mathbf{u}_{S}-\mathbf{u}_{S, h}\right|_{1, T_{S}}^{3}\right. \\
& \left.+\left\|\mathbf{u}_{D}-\mathbf{u}_{D, h}\right\|_{\mathbf{L}^{3}\left(T_{D}\right)}^{3}+h_{T_{D}}^{2}\left\|\operatorname{div}\left(\mathbf{u}_{D}-\mathbf{u}_{D, h}\right)\right\|_{0, T_{D}}^{3}\right\},
\end{aligned}
$$

where $T_{S}$ and $T_{D}$ are the triangles of $\mathcal{T}_{h}^{S}$ and $\mathcal{T}_{h}^{D}$, respectively, having e as an edge. 
Proof. Given $e \in \mathcal{E}_{h}(\Sigma)$, we let $T_{S}$ and $T_{D}$ be the triangles of $\mathcal{T}_{h}^{S}$ and $\mathcal{T}_{h}^{D}$, respectively, having $e$ as an edge, which means that $\omega_{e}:=T_{S} \cup T_{D}$, and define $\chi_{e}:=\mathbf{u}_{S, h} \cdot \mathbf{n}-\mathbf{u}_{D, h} \cdot \mathbf{n}$ on $e$. Then, applying (5.43) to $\left\|\chi_{e}\right\|_{L^{3}(e)}$, we have

$$
c_{2}\left\|\chi_{e}\right\|_{L^{3}(e)} \leq \sup _{\tau \in V_{e}} \frac{\int_{e} \chi_{e} \psi_{e} \tau}{\|\tau\|_{L^{3 / 2}(e)}} .
$$

Next, setting $\psi_{e, \star}:=\left.\psi_{e}\right|_{T_{\star}}$, with $\star \in\{S, D\}$, using the identity $\mathbf{u}_{D} \cdot \mathbf{n}=\mathbf{u}_{S} \cdot \mathbf{n}$ on $\Sigma$ (cf. Thm. 5.8), recalling that $\psi_{e, \star}=0$ on $\partial T_{\star} \backslash e$, and integrating by parts on $T_{\star}$, we obtain

$$
\begin{aligned}
\int_{e} \chi_{e} \psi_{e} \tau= & \int_{T_{S}}\left(\mathbf{u}_{S}-\mathbf{u}_{S, h}\right) \cdot \nabla\left(\psi_{e, S} L(\tau)\right)+\int_{T_{S}} \psi_{e, S} L(\tau) \operatorname{div}\left(\mathbf{u}_{S}-\mathbf{u}_{S, h}\right) \\
& +\int_{T_{D}}\left(\mathbf{u}_{D}-\mathbf{u}_{D, h}\right) \cdot \nabla\left(\psi_{e, D} L(\tau)\right)+\int_{T_{D}} \psi_{e, D} L(\tau) \operatorname{div}\left(\mathbf{u}_{D}-\mathbf{u}_{D, h}\right) .
\end{aligned}
$$

Thus, using the Cauchy-Schwarz and Hölder inequalities, applying (5.45) to $\left\|\nabla\left(\psi_{e, \star} L(\tau)\right)\right\|_{\mathbf{L}^{3 / 2}\left(T_{\star}\right)}$, and utilizing the local inverse estimate $\left\|\psi_{e, \star} L(\tau)\right\|_{0, T_{\star}} \leq c h_{T_{\star}}^{-1 / 3}\left\|\psi_{e, \star} L(\tau)\right\|_{L^{3 / 2}\left(T_{\star}\right)}(c f .(5.47))$, and the fact that $0 \leq \psi_{e} \leq 1$ in $\omega_{e}$, we find that

$$
\begin{aligned}
\int_{e} \chi_{e} \psi_{e} \tau \leq & C\left\{h_{T_{S}}^{-1}\left\|\mathbf{u}_{S}-\mathbf{u}_{S, h}\right\|_{\mathbf{L}^{3}\left(T_{S}\right)}+h_{T_{S}}^{-1 / 3}\left\|\operatorname{div}\left(\mathbf{u}_{S}-\mathbf{u}_{S, h}\right)\right\|_{0, T_{S}}\right\}\left\|\psi_{e, S} L(\tau)\right\|_{L^{3 / 2}\left(T_{S}\right)} \\
& +C\left\{h_{T_{D}}^{-1}\left\|\mathbf{u}_{D}-\mathbf{u}_{D, h}\right\|_{\mathbf{L}^{3}\left(T_{D}\right)}+h_{T_{D}}^{-1 / 3}\left\|\operatorname{div}\left(\mathbf{u}_{D}-\mathbf{u}_{D, h}\right)\right\|_{0, T_{D}}\right\}\left\|\psi_{e, D} L(\tau)\right\|_{L^{3 / 2}\left(T_{D}\right)} .
\end{aligned}
$$

Finally, applying now (5.46) to $\left\|\psi_{e, \star} L(\tau)\right\|_{L^{3 / 2}\left(T_{\star}\right)}$, combining the resulting estimate with (5.58) and (5.57), and using that $h_{e} \leq h_{T_{\star}}$, we arrive at (5.56) and conclude the proof.

In order to complete the global efficiency given by (5.38) ( $c f$. Thm. 5.7), we now need to estimate the terms $\left\|\lambda-\lambda_{h}\right\|_{0, e}^{2},\left\|\mathbf{u}_{S}-\mathbf{u}_{S, h}\right\|_{\mathbf{L}^{3}(T)}^{3},\left\|\operatorname{div}\left(\mathbf{u}_{S}-\mathbf{u}_{S, h}\right) \mathbf{u}_{S, h}\right\|_{\mathbf{L}^{4 / 3}(T)}^{2},\left\|\left(\nabla \mathbf{u}_{S}\right) \mathbf{u}_{S}-\left(\nabla \mathbf{u}_{S, h}\right) \mathbf{u}_{S, h}\right\|_{\mathbf{L}^{4 / 3}(T)}^{2}$, and $\|\left|\mathbf{u}_{D}\right| \mathbf{u}_{D}-$ $\left|\mathbf{u}_{D, h}\right| \mathbf{u}_{D, h} \|_{\mathbf{L}^{3 / 2}(T)}^{3 / 2}$ appearing in the upper bounds provided by Lemmas 5.12-5.17. To this end, we first recall that $W^{1 / 3,3 / 2}(\Sigma)$ is continuously embedded into $L^{2}(\Sigma)$, whence

$$
\sum_{e \in \mathcal{E}_{h}(\Sigma)}\left\|\lambda-\lambda_{h}\right\|_{0, e}^{2} \leq\left\|\lambda-\lambda_{h}\right\|_{0, \Sigma}^{2} \leq C\left\|\lambda-\lambda_{h}\right\|_{1 / 3,3 / 2 ; \Sigma}^{2}
$$

In turn, we make use of the continuity of the injection $\mathbf{i}: \mathbf{H}^{1}\left(\Omega_{S}\right) \rightarrow \mathbf{L}^{3}\left(\Omega_{S}\right)$ to obtain

$$
\sum_{T \in \mathcal{T}_{h}^{S}}\left\|\mathbf{u}_{S}-\mathbf{u}_{S, h}\right\|_{\mathbf{L}^{3}(T)}^{3}=\left\|\mathbf{u}_{S}-\mathbf{u}_{S, h}\right\|_{\mathbf{L}^{3}\left(\Omega_{S}\right)}^{3} \leq C\left\|\mathbf{u}_{S}-\mathbf{u}_{S, h}\right\|_{1, \Omega_{S}}^{3} .
$$

In addition, applying Hölder's inequality with $p=3 / 2$ and $q=3$, we first obtain

$$
\left\|\operatorname{div}\left(\mathbf{u}_{S}-\mathbf{u}_{S, h}\right) \mathbf{u}_{S, h}\right\|_{\mathbf{L}^{4 / 3}(T)}^{2} \leq\left\|\mathbf{u}_{S, h}\right\|_{\mathbf{L}^{4}(T)}^{2}\left\|\operatorname{div}\left(\mathbf{u}_{S}-\mathbf{u}_{S, h}\right)\right\|_{0, T}^{2},
$$

and then, bounding $\left\|\mathbf{u}_{S, h}\right\|_{\mathbf{L}^{4}(T)}^{2}$ by $\left\|\mathbf{u}_{S, h}\right\|_{\mathbf{L}^{4}\left(\Omega_{S}\right)}^{2}$ for each $T \in \mathcal{T}_{h}^{S}$, and employing the continuous injection $\mathbf{i}: \mathbf{H}^{1}\left(\Omega_{S}\right) \rightarrow \mathbf{L}^{4}\left(\Omega_{S}\right)$, and the a priori bounds of $\left\|\mathbf{u}_{S, h}\right\|_{1, \Omega_{S}}(c f$. (5.15)) combined with (5.9), we deduce that

$$
\begin{aligned}
\sum_{T \in \mathcal{T}_{h}^{S}}\left\|\operatorname{div}\left(\mathbf{u}_{S}-\mathbf{u}_{S, h}\right) \mathbf{u}_{S, h}\right\|_{\mathbf{L}^{4 / 3}(T)}^{2} & \leq\left\|\mathbf{u}_{S, h}\right\|_{\mathbf{L}^{4}\left(\Omega_{S}\right)}^{2} \sum_{T \in \mathcal{T}_{h}^{S}}\left\|\operatorname{div}\left(\mathbf{u}_{S}-\mathbf{u}_{S, h}\right)\right\|_{0, T}^{2} \\
& \leq C\left\|\mathbf{u}_{S, h}\right\|_{1, \Omega_{S}}^{2}\left\|\mathbf{u}_{S}-\mathbf{u}_{S, h}\right\|_{1, \Omega_{S}}^{2} \leq C\left\|\mathbf{u}_{S}-\mathbf{u}_{S, h}\right\|_{1, \Omega_{S}}^{2} .
\end{aligned}
$$


Similarly, adding and subtracting $\left(\nabla \mathbf{u}_{S}\right) \mathbf{u}_{S, h}$ (it also works with $\left.\left(\nabla \mathbf{u}_{S, h}\right) \mathbf{u}_{S}\right)$, applying Hölder's inequality as in (5.61), and using again the continuous injection $\mathbf{i}: \mathbf{H}^{1}\left(\Omega_{S}\right) \rightarrow \mathbf{L}^{4}\left(\Omega_{S}\right)$, and the a priori bounds of $\left\|\mathbf{u}_{S}\right\|_{1, \Omega_{S}}$ and $\left\|\mathbf{u}_{S, h}\right\|_{1, \Omega_{S}}$ (cf. (5.15)) combined with (5.9), we are able to show that

$$
\sum_{T \in \mathcal{T}_{h}^{S}}\left\|\left(\nabla \mathbf{u}_{S}\right) \mathbf{u}_{S}-\left(\nabla \mathbf{u}_{S, h}\right) \mathbf{u}_{S, h}\right\|_{\mathbf{L}^{4 / 3}(T)}^{2} \leq C\left\|\mathbf{u}_{S}-\mathbf{u}_{S, h}\right\|_{1, \Omega_{S}}^{2} .
$$

Finally, thanks to the Cauchy-Schwarz inequality and the a priori bounds of $\left\|\mathbf{u}_{D}\right\|_{\mathbf{H}^{3}\left(\operatorname{div} ; \Omega_{D}\right)}$ and $\left\|\mathbf{u}_{D, h}\right\|_{\mathbf{H}^{3}\left(\operatorname{div} ; \Omega_{D}\right)}(c f .(5.15))$ combined with (5.9), we find that

$$
\begin{aligned}
\sum_{T \in \mathcal{T}_{h}^{D}}\left\|\left|\mathbf{u}_{D}\right| \mathbf{u}_{D}-\left|\mathbf{u}_{D, h}\right| \mathbf{u}_{D, h}\right\|_{\mathbf{L}^{3 / 2}(T)}^{3 / 2} & \leq C \sum_{T \in \mathcal{T}_{h}^{D}}\left\{\left\|\mathbf{u}_{D}\right\|_{\mathbf{L}^{3}(T)}^{3 / 2}+\left\|\mathbf{u}_{D, h}\right\|_{\mathbf{L}^{3}(T)}^{3 / 2}\right\}\left\|\mathbf{u}_{D}-\mathbf{u}_{D, h}\right\|_{\mathbf{L}^{3}(T)}^{3 / 2} \\
& \leq C\left\{\left\|\mathbf{u}_{D}\right\|_{\mathbf{L}^{3}\left(\Omega_{D}\right)}^{3 / 2}+\left\|\mathbf{u}_{D, h}\right\|_{\mathbf{L}^{3}\left(\Omega_{D}\right)}^{3 / 2}\right\}\left\|\mathbf{u}_{D}-\mathbf{u}_{D, h}\right\|_{\mathbf{L}^{3}\left(\Omega_{D}\right)}^{3 / 2} \\
& \leq C\left\|\mathbf{u}_{D}-\mathbf{u}_{D, h}\right\|_{\mathbf{H}^{3}\left(\operatorname{div} ; \Omega_{D}\right)}^{3 / 2} .
\end{aligned}
$$

Consequently, it is not difficult to see that (5.38) follows from the definition of $\Theta$ (cf. (5.7)), Lemmas 5.9, $5.12-5.17$, and the estimates (5.59)-(5.64).

\section{Numerical Results}

This section serves to illustrate the performance and accuracy of our mixed finite element scheme (3.3) along with the reliability and efficiency properties of the a posteriori error estimator $\Theta(c f .(5.7))$ in 2D domains derived in Section 5. Our implementation is based on a FreeFem ++ code [26]. Regarding the implementation of the Newton iterative method associated to (3.3) (see [11], Sect. 6 for details), the iterations are terminated once the relative error of the entire coefficient vectors between two consecutive iterates, say coeff $^{m}$ and coeff ${ }^{m+1}$, is sufficiently small, i.e.

$$
\frac{\| \text { coeff }^{m+1}-\text { coeff }^{m} \|_{\ell^{2}}}{\| \text { coeff }^{m+1} \|_{\ell^{2}}} \leq \text { tol }
$$

where $\|\cdot\|_{\ell^{2}}$ is the standard $\ell^{2}$-norm in $\mathbb{R}^{N}$, with $N$ denoting the total number of degrees of freedom defining the finite element subspaces $\mathbf{H}_{h}$ and $\mathbf{Q}_{h}$ (cf. Sect. 3.3), and tol is a fixed tolerance chosen as tol $=1 E-06$. As usual, the individual errors are denoted by:

$$
\begin{gathered}
\mathrm{e}\left(\mathbf{u}_{S}\right):=\left\|\mathbf{u}_{S}-\mathbf{u}_{S, h}\right\|_{1, \Omega_{S}}, \quad \mathrm{e}\left(p_{S}\right):=\left\|p_{S}-p_{S, h}\right\|_{0, \Omega_{S}}, \\
\mathrm{e}\left(\mathbf{u}_{D}\right):=\left\|\mathbf{u}_{D}-\mathbf{u}_{D, h}\right\|_{\mathbf{H}^{3}\left(\operatorname{div} ; \Omega_{D}\right)}, \quad \mathrm{e}\left(p_{D}\right):=\left\|p_{D}-p_{D, h}\right\|_{0, \Omega_{D}}, \quad \mathrm{e}(\lambda):=\left\|\lambda-\lambda_{h}\right\|_{L^{3 / 2}(\Sigma)} .
\end{gathered}
$$

Note here that $\left\|\lambda-\lambda_{h}\right\|_{1 / 3,3 / 2 ; \Sigma}$ has been replaced by $\left\|\lambda-\lambda_{h}\right\|_{L^{3 / 2}(\Sigma)}$ since $\|\cdot\|_{1 / 3,3 / 2 ; \Sigma}$ is not easily computable. Similarly, since it does not seem trivial to define an explicit lifting $\varphi_{h}$, in the numerical results reported below, and more precisely in the definition of $\widetilde{\Theta}_{D, T}^{3 / 2}\left(c f\right.$. (5.4)), we replace $\varphi_{h}$ by a computable approximation of it denoted $\psi_{h}$. In fact, observing that the efficiency estimate (5.38) suggests that $\varphi_{h}$ be as close as possible to $p_{D}$, we let $\psi_{h}$ be the unique function in the finite element subspace $X_{h}:=\left\{v \in \mathcal{C}\left(\bar{\Omega}_{D}\right):\left.\quad v\right|_{T} \in P_{1}(T) \quad \forall T \in \mathcal{T}_{h}^{D}\right\}$, whose value on each interior vertex $\mathbf{x}$ of the triangulation $\mathcal{T}_{h}^{D}$ is given by the average of the constant values of $p_{D, h}$ on the triangles to which $\mathbf{x}$ belongs. Similarly, for each vertex $\mathbf{x}$ lying on $\partial \Omega_{D}, \psi_{h}(\mathbf{x})$ is defined as the average of the constant values of $\hat{\lambda}_{h}$ on the edges to which $\mathbf{x}$ belongs. We stress here that, when replacing $\varphi_{h}$ by $\psi_{h}$ in (5.35), the consistency error generated in the estimation of $\mathcal{R}_{2}\left(\operatorname{curl} \widehat{\beta}_{D}\right)(c f .(5.36))$ is given by

$$
\left\langle\operatorname{curl} \widehat{\beta}_{D} \cdot \mathbf{n}, \widehat{\lambda}_{h}-\psi_{h}\right\rangle_{\partial \Omega_{D}},
$$


which, according to the duality between $W^{-1 / 3,3}\left(\partial \Omega_{D}\right)$ and $W^{1 / 3,3 / 2}\left(\partial \Omega_{D}\right)$, is first estimated as

$$
\left|\left\langle\operatorname{curl} \widehat{\beta}_{D} \cdot \mathbf{n}, \widehat{\lambda}_{h}-\psi_{h}\right\rangle_{\partial \Omega_{D}}\right| \leq\left\|\operatorname{curl} \widehat{\beta}_{D} \cdot \mathbf{n}\right\|_{-1 / 3,3 ; \partial \Omega_{D}}\left\|\widehat{\lambda}_{h}-\psi_{h}\right\|_{1 / 3,3 / 2 ; \partial \Omega_{D}} .
$$

Then, employing the boundedness of the normal trace of $\mathbf{H}^{3}\left(\operatorname{div}_{3} ; \Omega_{D}\right)$ ( $c f .(4.12)$ and [16], Cor. B.57), the boundedness of the Clément interpolator $I_{h}^{D}: W^{1,3}\left(\Omega_{D}\right) \rightarrow W^{1,3}\left(\Omega_{D}\right)$ (cf. [16], Lem. 1.127, p. 69), and the stability estimate (5.28), we find that

$$
\begin{aligned}
\left\|\operatorname{curl} \widehat{\beta}_{D} \cdot \mathbf{n}\right\|_{-1 / 3,3 ; \partial \Omega_{D}} & \leq C\left\|\operatorname{curl} \widehat{\beta}_{D}\right\|_{\mathbf{H}^{3}\left(\operatorname{div}_{3} ; \Omega_{D}\right)}=C\left\|\operatorname{curl} \widehat{\beta}_{D}\right\|_{L^{3}\left(\Omega_{D}\right)} \\
& \leq C\left\|\widehat{\beta}_{D}\right\|_{1,3 ; \Omega_{D}} \leq C\left\|\beta_{D}\right\|_{1,3 ; \Omega_{D}} \leq C\left\|\mathbf{v}_{D}\right\|_{\mathbf{H}^{3}\left(\operatorname{div} ; \Omega_{D}\right)},
\end{aligned}
$$

which, replaced back into (6.1), yields

$$
\left|\left\langle\operatorname{curl} \widehat{\beta}_{D} \cdot \mathbf{n}, \widehat{\lambda}_{h}-\psi_{h}\right\rangle_{\partial \Omega_{D}}\right| \leq C\left\|\mathbf{v}_{D}\right\|_{\mathbf{H}^{3}\left(\operatorname{div} ; \Omega_{D}\right)}\left\|\widehat{\lambda}_{h}-\psi_{h}\right\|_{1 / 3,3 / 2 ; \partial \Omega_{D}}
$$

The foregoing inequality shows that our consistency error is under control as long as $\psi_{h}$ constitutes a good approximation of $\widehat{\lambda}_{h}$. Regarding this fact, in the examples reported below we provide numerical evidence that this is indeed the case, at least in the $L^{3 / 2}\left(\partial \Omega_{D}\right)$-norm, by computing

$$
\mathrm{e}\left(\widehat{\lambda}_{h}\right):=\left\|\widehat{\lambda}_{h}-\psi_{h}\right\|_{L^{3 / 2}\left(\partial \Omega_{D}\right)}
$$

and its corresponding rate of convergence, which is observed to behave of $O(h)$ in all cases. In turn, the global error is computed as $\mathrm{e}(\overrightarrow{\mathbf{u}})+\mathrm{e}\left(\psi_{h}\right)$, where

$$
\mathrm{e}(\overrightarrow{\mathbf{u}}):=\mathrm{e}\left(\mathbf{u}_{S}\right)+\mathrm{e}\left(\mathbf{u}_{D}\right)+\mathrm{e}\left(p_{S}\right)+\mathrm{e}\left(p_{D}\right)+\mathrm{e}(\lambda) \text { and } \mathrm{e}\left(\psi_{h}\right):=\left|p_{D}-\psi_{h}\right|_{1,3 / 2 ; \Omega_{D}},
$$

whereas the efficiency and reliability indexes with respect to $\Theta$ are given by

$$
\operatorname{eff}(\Theta):=\frac{\mathrm{e}(\overrightarrow{\mathbf{u}})+\mathrm{e}\left(\psi_{h}\right)}{\Theta} \text { and } \operatorname{rel}\left(\Theta^{1 / 2}\right):=\frac{\mathrm{e}(\overrightarrow{\mathbf{u}})}{\Theta^{1 / 2}} .
$$

Regarding these indexes, and assuming that $\mathrm{e}\left(\psi_{h}\right) \leq \mathrm{e}(\overrightarrow{\mathbf{u}})$, which is attained in all the numerical essays below, we observe from (5.8) (after discarding the higher order terms there) that

$$
C_{\text {eff }} \leq \operatorname{eff}(\Theta) \leq 2 C_{\mathrm{rel}} \Theta^{-1 / 2} \text { and } \frac{C_{\text {eff }}}{2} \Theta^{1 / 2} \leq \operatorname{rel}\left(\Theta^{1 / 2}\right) \leq C_{\mathrm{rel}},
$$

which says that, while eff $(\Theta)$ and $\operatorname{rel}\left(\Theta^{1 / 2}\right)$ are below and above bounded, respectively, eff $(\Theta)$ could become above unbounded whereas rel $\left(\Theta^{1 / 2}\right)$ could very well approaches 0 as $\Theta$ goes to 0. Nevertheless, the numerical results to be displayed below show that $\operatorname{eff}(\Theta)$ remains always above bounded as well, whereas $\operatorname{rel}\left(\Theta^{1 / 2}\right)$ does in fact decreases as $\Theta$ goes to 0 .

In addition, we define the experimental rates of convergence

$$
\mathrm{r}(\diamond):=\frac{\log \left(\mathrm{e}(\diamond) / \mathrm{e}^{\prime}(\diamond)\right)}{\log \left(h / h^{\prime}\right)} \quad \text { for each } \diamond \in\left\{\mathbf{u}_{S}, \mathbf{u}_{D}, p_{S}, p_{D}, \lambda, \widehat{\lambda}_{h}, \overrightarrow{\mathbf{u}}\right\},
$$

where $h$ and $h^{\prime}$ denote two consecutive mesh sizes, taken accordingly from $\left\{h_{S}, h_{D}, h_{\Sigma}\right\}$, with their respective errors $e$ and $e^{\prime}$. However, when the adaptive algorithm is applied, the expression $\log \left(h / h^{\prime}\right)$ appearing in the computation of the above rates is replaced by $-\frac{1}{2} \log \left(N / N^{\prime}\right)$, where $N$ and $N^{\prime}$ denote the corresponding degrees of freedom of each triangulation.

The examples to be considered in this section are described next. In all of them, for the sake of simplicity, we choose the parameters $\mu=1, \rho=1, \mathbf{F}=1, \alpha_{d}=1, \boldsymbol{\kappa}=\mathbb{I}$, and $\mathbf{K}=\mathbb{I}$. Furthermore, the condition $\left(p_{h}, 1\right)_{\Omega}=0$ is imposed via a penalization strategy.

Example 1 is used to corroborate the reliability and efficiency of the a posteriori error estimator $\Theta$, whereas Example 2 is utilized to illustrate the behavior of the associated adaptive algorithm, which applies the following procedure from [30]: 
(1) Start with a coarse mesh $\mathcal{T}_{h}:=\mathcal{T}_{h}^{S} \cup \mathcal{T}_{h}^{D}$.

(2) Solve the Newton iterative method associated to (3.3) for the current mesh $\mathcal{T}_{h}$.

(3) Compute the local indicator $\Theta_{T}$ for each $T \in \mathcal{T}_{h}:=\mathcal{T}_{h}^{S} \cup \mathcal{T}_{h}^{D}$ and $e \in \mathcal{E}_{h}(\Sigma)$, where

$$
\Theta_{T}:=\left\{\Theta_{S, T}^{2}+\widehat{\Theta}_{D, T}^{2}\right\}^{1 / 2}+\widetilde{\Theta}_{D, T}+\Theta_{\Sigma, e}, \quad(c f .(5.1),(5.3),(5.4),(5.6)) .
$$

(4) Check the stopping criterion and decide whether to finish or go to next step.

(5) Use the automatic meshing algorithm adaptmesh from Section 9.1.9 of [27] to refine each $T^{\prime} \in \mathcal{T}_{h}$ satisfying:

$$
\Theta_{T^{\prime}} \geq C_{\mathrm{adm}} \frac{1}{\# T} \sum_{T \in \mathcal{T}_{h}} \Theta_{T}, \quad \text { for some } C_{\mathrm{adm}} \in(0,1),
$$

where \#T denotes the number of triangles of the mesh $\mathcal{T}_{h}$.

(6) Define resulting meshes as current meshes $\mathcal{T}_{h}^{S}$ and $\mathcal{T}_{h}^{D}$, and go to step (2).

In particular, in Example 2 below we take $C_{\mathrm{adm}}=0.8$. In turn, if the refinement is with respect to the local indicator $\Theta_{T}^{1 / 2}$, then we simply replace $\Theta_{T^{\prime}}$ and $\Theta_{T}$ by $\Theta_{T^{\prime}}^{1 / 2}$ and $\Theta_{T}^{1 / 2}$, respectively, in the criterion (6.4).

\section{Example 1: Accuracy assessment with a smooth solution in a rectangular domain.}

In our first example we consider a rectangle domain divided in two coupled squares, i.e. $\Omega_{S}:=(0,1) \times(1,2)$, $\Omega_{D}:=(0,1)^{2}$ and $\Sigma:=(0,1) \times\{1\}$. The data $\mathbf{f}_{S}, \mathbf{f}_{D}$, and $g_{D}$ are chosen so that the exact solution in the rectangle domain $\Omega=\Omega_{S} \cup \Sigma \cup \Omega_{D}$ is given by the smooth functions

$$
\begin{aligned}
& \mathbf{u}_{S}:=\left(\begin{array}{c}
-2 \sin \left(\pi x_{1}\right) \cos \left(2 \pi x_{2}\right) \\
\cos \left(\pi x_{1}\right) \sin \left(2 \pi x_{2}\right)
\end{array}\right), \quad \mathbf{u}_{D}:=\left(\begin{array}{c}
\sin \left(\pi x_{1}\right) \exp \left(x_{2}\right) \\
\sin \left(\pi x_{2}\right) \exp \left(x_{1}\right)
\end{array}\right), \\
& p_{\star}:=\frac{1}{5} x_{1} \cos \left(\pi x_{2}\right) \quad \text { in } \quad \Omega_{\star}, \quad \text { with } \star \in\{S, D\} .
\end{aligned}
$$

Notice that this solution satisfies $\mathbf{u}_{S} \cdot \mathbf{n}=\mathbf{u}_{D} \cdot \mathbf{n}$ on $\Sigma$. However, the Beavers-Joseph-Saffman condition (2.3) is not satisfied, whereas the Dirichlet boundary condition for the Navier-Stokes velocity on $\Gamma_{S}$ and the Neumann boundary condition for the Darcy-Forchheimer velocity on $\Gamma_{D}$ are both non-homogeneous. In this way, the

\begin{tabular}{|c|c|c|c|c|c|c|c|c|c|c|c|}
\hline$N$ & $h_{S}$ & $h_{D}$ & Iter & $\mathrm{e}\left(\mathbf{u}_{S}\right)$ & $\mathbf{r}\left(\mathbf{u}_{S}\right)$ & $\mathrm{e}\left(p_{S}\right)$ & $\mathrm{r}\left(p_{S}\right)$ & $\mathrm{e}\left(\mathbf{u}_{D}\right)$ & $r\left(\mathbf{u}_{D}\right)$ & $\mathrm{e}\left(p_{D}\right)$ & $\mathbf{r}\left(p_{D}\right)$ \\
\hline 279 & 0.373 & 0.373 & 5 & $2.39 \mathrm{e}+00$ & - & $9.05 \mathrm{e}-01$ & - & $1.14 \mathrm{e}+00$ & - & $4.22 \mathrm{e}-02$ & - \\
\hline 1061 & 0.196 & 0.190 & 5 & $1.01 \mathrm{e}+00$ & 1.339 & $2.92 \mathrm{e}-01$ & 1.766 & $5.31 \mathrm{e}-01$ & 1.133 & $1.48 \mathrm{e}-02$ & 1.557 \\
\hline 3877 & 0.103 & 0.097 & 5 & $4.84 \mathrm{e}-01$ & 1.135 & $1.44 \mathrm{e}-01$ & 1.089 & $2.62 \mathrm{e}-01$ & 1.043 & $4.95 \mathrm{e}-03$ & 1.617 \\
\hline 15057 & 0.051 & 0.057 & 5 & $2.52 \mathrm{e}-01$ & 0.929 & $6.70 \mathrm{e}-02$ & 1.090 & $1.35 \mathrm{e}-01$ & 1.268 & $2.17 \mathrm{e}-03$ & 1.579 \\
\hline 59203 & 0.027 & 0.026 & 5 & $1.23 \mathrm{e}-01$ & 1.144 & $3.81 \mathrm{e}-02$ & 0.898 & $6.73 \mathrm{e}-02$ & 0.877 & $1.01 \mathrm{e}-03$ & 0.967 \\
\hline 236687 & 0.014 & 0.013 & 5 & $6.12 \mathrm{e}-02$ & 1.004 & $1.79 \mathrm{e}-02$ & 1.092 & $3.35 \mathrm{e}-02$ & 1.068 & $4.93 \mathrm{e}-04$ & 1.091 \\
\hline$h_{\Sigma}$ & $\mathrm{e}(\lambda)$ & $r(\lambda)$ & $\mathrm{e}\left(\widehat{\lambda}_{h}\right)$ & $\mathrm{r}(\widehat{\lambda}$ & $\mathrm{e}(\overrightarrow{\mathbf{u}})$ & 10 & $\mathrm{e}\left(\psi_{h}\right)$ & $\Theta$ & eff $(\Theta)$ & \multicolumn{2}{|c|}{$\operatorname{rel}\left(\Theta^{1 / 2}\right)$} \\
\hline $1 / 4$ & $4.52 \mathrm{e}-02$ & - & $6.84 \mathrm{e}-02$ & - & $4.52 \mathrm{e}+00$ & - & $1.69 \mathrm{e}-01$ & $2.38 \mathrm{e}+01$ & 0.1971 & \multicolumn{2}{|c|}{0.9267} \\
\hline $1 / 8$ & $1.38 \mathrm{e}-02$ & 1.712 & $2.20 \mathrm{e}-02$ & 1.635 & $1.86 \mathrm{e}+00$ & 1.382 & $8.51 \mathrm{e}-02$ & $9.86 \mathrm{e}+00$ & 0.1977 & \multicolumn{2}{|c|}{0.5938} \\
\hline $1 / 16$ & $4.94 \mathrm{e}-03$ & 1.480 & $1.04 \mathrm{e}-02$ & 1.085 & $9.00 \mathrm{e}-01$ & 1.120 & $4.92 \mathrm{e}-02$ & $5.04 \mathrm{e}+00$ & 0.1884 & \multicolumn{2}{|c|}{0.4011} \\
\hline $1 / 32$ & $1.86 \mathrm{e}-03$ & 1.414 & $5.04 \mathrm{e}-03$ & 1.044 & $4.59 \mathrm{e}-01$ & 1.160 & $4.28 \mathrm{e}-02$ & $2.56 \mathrm{e}+00$ & 0.1956 & \multicolumn{2}{|c|}{0.2864} \\
\hline $1 / 64$ & $8.53 \mathrm{e}-04$ & 1.121 & $2.49 \mathrm{e}-03$ & 1.014 & $2.30 \mathrm{e}-01$ & 0.921 & $4.00 \mathrm{e}-02$ & $1.32 \mathrm{e}+00$ & 0.2040 & \multicolumn{2}{|c|}{0.2001} \\
\hline $1 / 128$ & $4.15 \mathrm{e}-04$ & 1.041 & $1.24 \mathrm{e}-03$ & 1.009 & $1.14 \mathrm{e}-01$ & 1.018 & $3.84 \mathrm{e}-02$ & $6.70 \mathrm{e}-01$ & 0.2266 & \multicolumn{2}{|c|}{0.1386} \\
\hline
\end{tabular}
right-hand side of the resulting system must be modified accordingly as well as the global estimator $\Theta$ ( $c f$. (5.7)). The results reported in Table 1 are in accordance with the theoretical sub-optimal rate of convergence

Table 1. Example $1, \mathbf{B R}-\mathbf{R T}_{0}-P_{0}-P_{0}$ primal-mixed scheme with quasi-uniform refinement. 


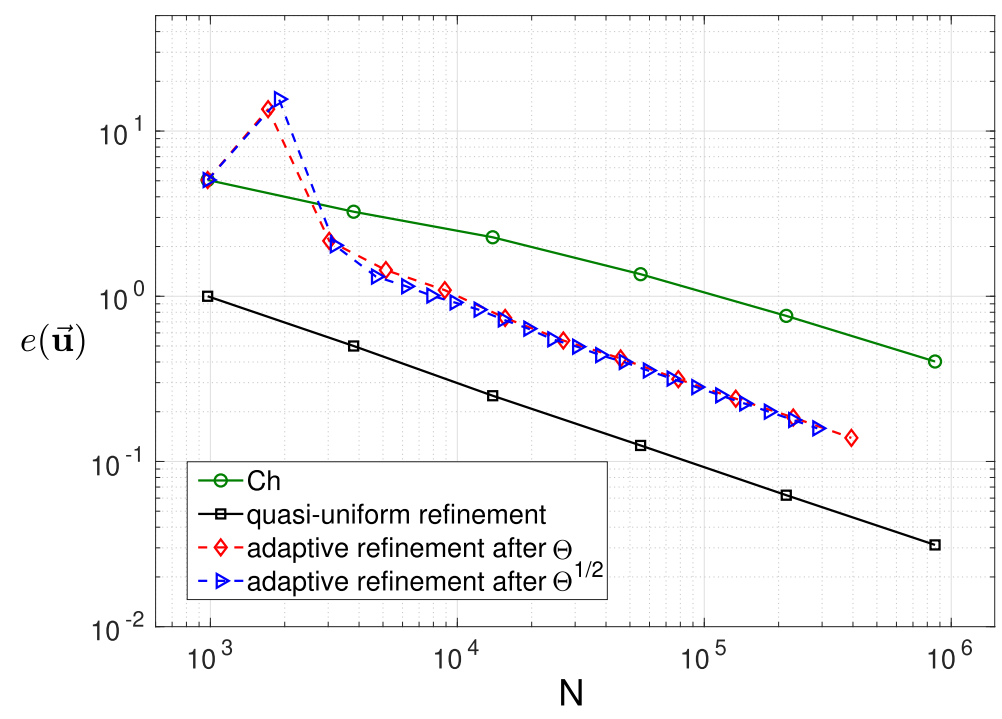

Figure 2. Example 2, Log-log plot of e $(\overrightarrow{\mathbf{u}})$ vs. $N$ for quasi-uniform/adaptive primal-mixed scheme.

TAble 2. Example 2, BR $-\mathbf{R T}_{0}-P_{0}-P_{0}$ primal-mixed scheme with quasi-uniform refinement.

\begin{tabular}{|c|c|c|c|c|c|c|c|c|c|c|c|}
\hline$N$ & $h_{S}$ & $h_{D}$ & Iter & $\mathrm{e}\left(\mathbf{u}_{S}\right)$ & $r\left(\mathbf{u}_{S}\right)$ & $\mathrm{e}\left(p_{S}\right)$ & $\mathrm{r}\left(p_{S}\right)$ & $\mathrm{e}\left(\mathbf{u}_{D}\right)$ & $r\left(\mathbf{u}_{D}\right)$ & $\mathrm{e}\left(p_{D}\right)$ & $\mathrm{r}\left(p_{D}\right)$ \\
\hline 975 & 0.188 & 0.200 & 4 & $2.90 \mathrm{e}+00$ & - & $1.47 \mathrm{e}+00$ & - & $6.38 \mathrm{e}-01$ & - & $2.80 \mathrm{e}-02$ & - \\
\hline 3803 & 0.100 & 0.095 & 5 & $2.13 \mathrm{e}+00$ & 0.495 & $7.99 \mathrm{e}-01$ & 0.976 & $3.07 \mathrm{e}-01$ & 0.984 & $1.36 \mathrm{e}-02$ & 0.974 \\
\hline 13907 & 0.050 & 0.049 & 5 & $1.56 \mathrm{e}+00$ & 0.450 & $5.56 \mathrm{e}-01$ & 0.523 & $1.56 \mathrm{e}-01$ & 1.037 & $6.76 \mathrm{e}-03$ & 1.065 \\
\hline 55232 & 0.026 & 0.026 & 5 & $8.94 \mathrm{e}-01$ & 0.837 & $3.84 \mathrm{e}-01$ & 0.559 & $7.83 e-02$ & 1.081 & $3.38 \mathrm{e}-03$ & 1.089 \\
\hline 214793 & 0.014 & 0.013 & 5 & $5.26 \mathrm{e}-01$ & 0.907 & $1.94 \mathrm{e}-01$ & 1.173 & $3.92 \mathrm{e}-02$ & 0.968 & $1.70 \mathrm{e}-03$ & 0.966 \\
\hline 859813 & 0.007 & 0.007 & 5 & $2.76 \mathrm{e}-01$ & 0.868 & $1.07 \mathrm{e}-01$ & 0.799 & $1.96 \mathrm{e}-02$ & 1.204 & $8.51 \mathrm{e}-04$ & 1.202 \\
\hline$h_{\Sigma}$ & $\mathrm{e}(\lambda)$ & $r(\lambda)$ & $\mathrm{e}\left(\widehat{\lambda}_{h}\right)$ & $r\left(\widehat{\lambda}_{h}\right)$ & $\mathrm{e}(\overrightarrow{\mathbf{u}})$ & $r(\overrightarrow{\mathbf{u}})$ & $\mathrm{e}\left(\psi_{h}\right)$ & $\Theta$ & $\operatorname{eff}(\Theta)$ & \multicolumn{2}{|c|}{$\operatorname{rel}\left(\Theta^{1 / 2}\right)$} \\
\hline $1 / 8$ & $1.45 \mathrm{e}-02$ & - & $6.89 \mathrm{e}-02$ & - & $5.06 \mathrm{e}+00$ & - & $2.64 \mathrm{e}-01$ & $2.84 \mathrm{e}+01$ & 0.1873 & \multicolumn{2}{|c|}{0.9486} \\
\hline $1 / 16$ & $3.65 \mathrm{e}-03$ & 1.993 & $3.26 \mathrm{e}-02$ & 1.081 & $3.25 \mathrm{e}+00$ & 0.650 & $2.14 \mathrm{e}-01$ & $2.54 \mathrm{e}+01$ & 0.1363 & \multicolumn{2}{|c|}{0.6447} \\
\hline $1 / 32$ & $8.87 \mathrm{e}-04$ & 2.042 & $1.57 \mathrm{e}-02$ & 1.053 & $2.28 \mathrm{e}+00$ & 0.549 & $1.66 \mathrm{e}-01$ & $1.32 \mathrm{e}+01$ & 0.1854 & \multicolumn{2}{|c|}{0.6269} \\
\hline $1 / 64$ & $4.78 \mathrm{e}-04$ & 0.893 & $7.85 \mathrm{e}-03$ & 0.999 & $1.36 \mathrm{e}+00$ & 0.746 & $1.73 \mathrm{e}-01$ & $8.13 \mathrm{e}+00$ & 0.1885 & \multicolumn{2}{|c|}{0.4769} \\
\hline $1 / 128$ & $4.74 \mathrm{e}-05$ & 3.334 & $3.88 \mathrm{e}-03$ & 1.018 & $7.61 \mathrm{e}-01$ & 0.855 & $1.57 \mathrm{e}-01$ & $4.78 \mathrm{e}+00$ & 0.1920 & \multicolumn{2}{|c|}{0.3480} \\
\hline $1 / 256$ & $2.90 \mathrm{e}-05$ & 0.705 & $1.94 \mathrm{e}-03$ & 1.000 & $4.03 \mathrm{e}-01$ & 0.917 & $1.56 \mathrm{e}-01$ & $2.43 \mathrm{e}+00$ & 0.2303 & \multicolumn{2}{|c|}{0.2584} \\
\hline
\end{tabular}

$O\left(h^{1 / 3}\right)$ provided by Theorem 5.2 of [11]. Actually, they are better than expected since they suggest that only technical difficulties stop us of proving an optimal rate of convergence $O(h)$, which is in fact observed there. In addition, we remark that the behaviors predicted by (6.3) and the comments right after it, are also illustrated in the table, in the sense that the efficiency index remains above and below bounded and the reliability index, while bounded as well, decreases as the estimators approach 0. Notice also that e $\left(\widehat{\lambda}_{h}\right)$ decreases with $O(h)$, which supports the choice of $\psi_{h}$ as an approximation of $\hat{\lambda}_{h}$ on $\partial \Omega_{D}$.

\section{Example 2: Adaptivity in a 2D helmet-shaped domain.}

In our second example, we consider a 2D helmet-shaped domain. More precisely, we consider the domain $\Omega=\Omega_{S} \cup \Sigma \cup \Omega_{D}$, where $\Omega_{S}:=(-1,-0.75) \times(0,1.25) \cup(-0.75,0.75) \times(0,0.25) \cup(0.75,1) \times(0,1.25), \Omega_{D}:=$ $(-1,1) \times(-0.5,0)$ and $\Sigma:=(-1,1) \times\{0\}$. The data $\mathbf{f}_{S}, \mathbf{f}_{D}$, and $g_{D}$ are chosen so that the exact solution in the 
Table 3. Example 2, BR $-\mathbf{R T}_{0}-P_{0}-P_{0}$ primal-mixed scheme with adaptive refinement via $\Theta$.

\begin{tabular}{|c|c|c|c|c|c|c|c|c|c|}
\hline$N$ & Iter & $\mathrm{e}\left(\mathbf{u}_{S}\right)$ & $r\left(\mathbf{u}_{S}\right)$ & $\mathrm{e}\left(p_{S}\right)$ & $\mathrm{r}\left(p_{S}\right)$ & $\mathrm{e}\left(\mathbf{u}_{D}\right)$ & $r\left(\mathbf{u}_{D}\right)$ & $\mathrm{e}\left(p_{D}\right)$ & $\mathrm{r}\left(p_{D}\right)$ \\
\hline 975 & 4 & $2.90 \mathrm{e}+00$ & - & $1.47 \mathrm{e}+00$ & - & $6.38 \mathrm{e}-01$ & - & $2.80 \mathrm{e}-02$ & - \\
\hline 1713 & 4 & $1.62 \mathrm{e}+00$ & 2.078 & $3.23 \mathrm{e}+00$ & - & $6.07 \mathrm{e}-01$ & 0.176 & $3.14 \mathrm{e}+00$ & - \\
\hline 3029 & 5 & $9.10 \mathrm{e}-01$ & 2.013 & $4.61 \mathrm{e}-01$ & 6.828 & $6.13 \mathrm{e}-01$ & - & $7.59 \mathrm{e}-02$ & 13.063 \\
\hline 5137 & 5 & $4.42 \mathrm{e}-01$ & 2.737 & $2.08 \mathrm{e}-01$ & 3.008 & $6.09 \mathrm{e}-01$ & 0.022 & $6.98 \mathrm{e}-02$ & 0.316 \\
\hline 8899 & 5 & $3.20 \mathrm{e}-01$ & 1.173 & $1.31 \mathrm{e}-01$ & 1.702 & $5.41 \mathrm{e}-01$ & 0.429 & $4.05 \mathrm{e}-02$ & 1.984 \\
\hline 15622 & 5 & $2.38 \mathrm{e}-01$ & 1.059 & $8.45 \mathrm{e}-02$ & 1.549 & $3.90 \mathrm{e}-01$ & 1.166 & $2.06 \mathrm{e}-02$ & 2.398 \\
\hline 26868 & 5 & $1.80 \mathrm{e}-01$ & 1.016 & $5.58 \mathrm{e}-02$ & 1.528 & $2.84 \mathrm{e}-01$ & 1.170 & $1.49 \mathrm{e}-02$ & 1.204 \\
\hline 45834 & 5 & $1.38 \mathrm{e}-01$ & 1.000 & $4.33 \mathrm{e}-02$ & 0.954 & $2.26 \mathrm{e}-01$ & 0.859 & $1.20 \mathrm{e}-02$ & 0.815 \\
\hline 78490 & 5 & $1.04 \mathrm{e}-01$ & 1.041 & $3.14 \mathrm{e}-02$ & 1.195 & $1.70 \mathrm{e}-01$ & 1.066 & $8.85 e-03$ & 1.121 \\
\hline 134124 & 5 & $7.98 \mathrm{e}-02$ & 1.002 & $2.30 \mathrm{e}-02$ & 1.164 & $1.30 \mathrm{e}-01$ & 0.984 & $6.49 \mathrm{e}-03$ & 1.159 \\
\hline 229268 & 5 & $6.09 \mathrm{e}-02$ & 1.006 & $1.77 \mathrm{e}-02$ & 0.980 & $1.00 \mathrm{e}-01$ & 0.971 & $5.34 \mathrm{e}-03$ & 0.727 \\
\hline 394144 & 5 & $4.67 \mathrm{e}-02$ & 0.986 & $1.34 \mathrm{e}-02$ & 1.023 & $7.49 \mathrm{e}-02$ & 1.081 & $3.84 \mathrm{e}-03$ & 1.215 \\
\hline $\mathrm{e}(\lambda)$ & $r(\lambda)$ & $\mathrm{e}\left(\widehat{\lambda}_{h}\right)$ & $r\left(\widehat{\lambda}_{h}\right)$ & $\mathrm{e}(\overrightarrow{\mathbf{u}})$ & $\mathrm{r}(\overrightarrow{\mathbf{u}})$ & $\mathrm{e}\left(\psi_{h}\right)$ & $\Theta$ & \multicolumn{2}{|c|}{$\operatorname{eff}(\Theta)$} \\
\hline $1.45 \mathrm{e}-02$ & - & $6.89 \mathrm{e}-02$ & - & $5.06 \mathrm{e}+00$ & - & $2.64 \mathrm{e}-01$ & $2.84 \mathrm{e}+01$ & \multicolumn{2}{|c|}{0.1873} \\
\hline $4.98 \mathrm{e}+00$ & - & $6.48 \mathrm{e}-02$ & 0.216 & $1.36 \mathrm{e}+01$ & - & $2.44 \mathrm{e}-01$ & $1.22 \mathrm{e}+01$ & \multicolumn{2}{|c|}{1.1288} \\
\hline $1.04 \mathrm{e}-01$ & 13.566 & $6.44 \mathrm{e}-02$ & 0.017 & $2.16 \mathrm{e}+00$ & 6.441 & $2.42 \mathrm{e}-01$ & $6.21 \mathrm{e}+00$ & \multicolumn{2}{|c|}{0.3875} \\
\hline $1.11 \mathrm{e}-01$ & - & $6.45 \mathrm{e}-02$ & - & $1.44 \mathrm{e}+00$ & 1.542 & $2.37 \mathrm{e}-01$ & $3.84 \mathrm{e}+00$ & \multicolumn{2}{|c|}{0.4367} \\
\hline $5.62 \mathrm{e}-02$ & 2.478 & $6.39 \mathrm{e}-02$ & 0.035 & $1.09 \mathrm{e}+00$ & 1.018 & $2.32 \mathrm{e}-01$ & $2.93 \mathrm{e}+00$ & \multicolumn{2}{|c|}{0.4508} \\
\hline $6.53 \mathrm{e}-03$ & 7.648 & $4.88 \mathrm{e}-02$ & 0.956 & $7.39 \mathrm{e}-01$ & 1.376 & $1.90 \mathrm{e}-01$ & $2.16 \mathrm{e}+00$ & \multicolumn{2}{|c|}{0.4298} \\
\hline $4.67 \mathrm{e}-03$ & 1.238 & $3.81 \mathrm{e}-02$ & 0.910 & $5.40 \mathrm{e}-01$ & 1.160 & $1.45 \mathrm{e}-01$ & $1.64 \mathrm{e}+00$ & \multicolumn{2}{|c|}{0.4187} \\
\hline $1.22 \mathrm{e}-03$ & 5.016 & $3.10 \mathrm{e}-02$ & 0.776 & $4.20 \mathrm{e}-01$ & 0.936 & $1.41 \mathrm{e}-01$ & $1.29 \mathrm{e}+00$ & \multicolumn{2}{|c|}{0.4365} \\
\hline $7.86 \mathrm{e}-04$ & 1.643 & $2.10 \mathrm{e}-02$ & 1.444 & $3.15 \mathrm{e}-01$ & 1.074 & $1.20 \mathrm{e}-01$ & $9.88 \mathrm{e}-01$ & \multicolumn{2}{|c|}{0.4403} \\
\hline $3.80 \mathrm{e}-04$ & 2.710 & $1.52 \mathrm{e}-02$ & 1.214 & $2.40 \mathrm{e}-01$ & 1.016 & $1.02 \mathrm{e}-01$ & $7.61 \mathrm{e}-01$ & \multicolumn{2}{|c|}{0.4489} \\
\hline $2.19 \mathrm{e}-04$ & 2.060 & $1.24 \mathrm{e}-02$ & 0.765 & $1.85 \mathrm{e}-01$ & 0.978 & $8.94 \mathrm{e}-02$ & $5.96 \mathrm{e}-01$ & \multicolumn{2}{|c|}{0.4596} \\
\hline $1.11 \mathrm{e}-04$ & 2.524 & $9.04 \mathrm{e}-03$ & 1.160 & $1.39 \mathrm{e}-01$ & 1.049 & $8.55 \mathrm{e}-02$ & $4.72 \mathrm{e}-01$ & \multicolumn{2}{|c|}{0.4754} \\
\hline
\end{tabular}

Table 4. Example 2, BR $-\mathbf{R T}_{0}-P_{0}-P_{0}$ primal-mixed scheme with adaptive refinement via $\Theta^{1 / 2}$, we show results for the meshes 1-2-3-4, 11-12-13-14, 19-20-21-22.

\begin{tabular}{|c|c|c|c|c|c|c|c|c|c|}
\hline$N$ & Iter & $\mathrm{e}\left(\mathbf{u}_{S}\right)$ & $r\left(\mathbf{u}_{S}\right)$ & $\mathrm{e}\left(p_{S}\right)$ & $\mathrm{r}\left(p_{S}\right)$ & $\mathrm{e}\left(\mathbf{u}_{D}\right)$ & $r\left(\mathbf{u}_{D}\right)$ & $\mathrm{e}\left(p_{D}\right)$ & $\mathrm{r}\left(p_{D}\right)$ \\
\hline 975 & 4 & $2.90 \mathrm{e}+00$ & - & $1.47 \mathrm{e}+00$ & - & $6.38 \mathrm{e}-01$ & - & $2.80 \mathrm{e}-02$ & - \\
\hline 1896 & 4 & $1.64 \mathrm{e}+00$ & 1.711 & $3.73 \mathrm{e}+00$ & - & $6.17 \mathrm{e}-01$ & 0.099 & $3.72 \mathrm{e}+00$ & - \\
\hline 3191 & 5 & $9.04 \mathrm{e}-01$ & 2.295 & $3.92 \mathrm{e}-01$ & 8.651 & $6.10 \mathrm{e}-01$ & 0.046 & $5.08 \mathrm{e}-02$ & 16.499 \\
\hline 4685 & 5 & $4.70 \mathrm{e}-01$ & 3.406 & $1.91 \mathrm{e}-01$ & 3.750 & $5.90 \mathrm{e}-01$ & 0.171 & $3.09 \mathrm{e}-02$ & 2.590 \\
\hline 24245 & 5 & $1.80 \mathrm{e}-01$ & 1.104 & $5.93 \mathrm{e}-02$ & 1.160 & $2.93 \mathrm{e}-01$ & 1.368 & $1.48 \mathrm{e}-02$ & 1.269 \\
\hline 30562 & 5 & $1.60 \mathrm{e}-01$ & 1.035 & $5.36 \mathrm{e}-02$ & 0.876 & $2.67 \mathrm{e}-01$ & 0.807 & $1.33 \mathrm{e}-02$ & 0.931 \\
\hline 37893 & 5 & $1.43 \mathrm{e}-01$ & 1.017 & $4.72 \mathrm{e}-02$ & 1.190 & $2.37 \mathrm{e}-01$ & 1.107 & $1.18 \mathrm{e}-02$ & 1.098 \\
\hline 47209 & 5 & $1.29 \mathrm{e}-01$ & 0.960 & $4.16 \mathrm{e}-02$ & 1.143 & $2.18 \mathrm{e}-01$ & 0.763 & $1.10 \mathrm{e}-02$ & 0.674 \\
\hline 145102 & 5 & $7.28 \mathrm{e}-02$ & 0.990 & $2.30 \mathrm{e}-02$ & 1.053 & $1.22 \mathrm{e}-01$ & 0.999 & $6.32 \mathrm{e}-03$ & 0.917 \\
\hline 182543 & 5 & $6.45 \mathrm{e}-02$ & 1.044 & $2.04 \mathrm{e}-02$ & 1.028 & $1.09 \mathrm{e}-01$ & 0.977 & $5.62 \mathrm{e}-03$ & 1.014 \\
\hline 229100 & 5 & $5.74 \mathrm{e}-02$ & 1.027 & $1.82 \mathrm{e}-02$ & 1.000 & $9.75 \mathrm{e}-02$ & 1.005 & $4.93 \mathrm{e}-03$ & 1.164 \\
\hline 286931 & 5 & $5.11 \mathrm{e}-02$ & 1.031 & $1.62 \mathrm{e}-02$ & 1.033 & $8.67 \mathrm{e}-02$ & 1.039 & $4.46 \mathrm{e}-03$ & 0.889 \\
\hline$e(\lambda)$ & $\mathrm{r}(\lambda)$ & $\mathrm{e}\left(\widehat{\lambda}_{h}\right)$ & $r\left(\widehat{\lambda}_{h}\right)$ & $\mathrm{e}(\overrightarrow{\mathbf{u}})$ & $\mathrm{r}(\overrightarrow{\mathbf{u}})$ & $\mathrm{e}\left(\psi_{h}\right)$ & $\Theta^{1 / 2}$ & \multicolumn{2}{|c|}{$\operatorname{rel}\left(\Theta^{1 / 2}\right)$} \\
\hline $1.45 \mathrm{e}-02$ & - & $6.89 \mathrm{e}-02$ & - & $5.06 \mathrm{e}+00$ & - & $2.64 \mathrm{e}-01$ & $5.33 \mathrm{e}+00$ & \multicolumn{2}{|c|}{0.9486} \\
\hline $5.90 \mathrm{e}+00$ & - & $6.49 \mathrm{e}-02$ & 0.179 & $1.56 \mathrm{e}+01$ & - & $2.43 \mathrm{e}-01$ & $3.53 \mathrm{e}+00$ & \multicolumn{2}{|c|}{4.4162} \\
\hline $7.72 \mathrm{e}-02$ & 16.656 & $6.38 \mathrm{e}-02$ & 0.067 & $2.03 \mathrm{e}+00$ & 7.829 & $2.34 \mathrm{e}-01$ & $2.46 \mathrm{e}+00$ & \multicolumn{2}{|c|}{0.8279} \\
\hline $3.32 \mathrm{e}-02$ & 4.393 & $6.37 \mathrm{e}-02$ & 0.003 & $1.32 \mathrm{e}+00$ & 2.270 & $2.33 \mathrm{e}-01$ & $1.99 \mathrm{e}+00$ & \multicolumn{2}{|c|}{0.6603} \\
\hline $1.98 \mathrm{e}-03$ & 11.340 & $3.36 \mathrm{e}-02$ & 1.737 & $5.49 \mathrm{e}-01$ & 1.324 & $1.66 \mathrm{e}-01$ & $1.29 \mathrm{e}+00$ & \multicolumn{2}{|c|}{0.4241} \\
\hline $1.66 \mathrm{e}-03$ & 1.568 & $3.07 \mathrm{e}-02$ & 0.775 & $4.95 \mathrm{e}-01$ & 0.895 & $1.58 \mathrm{e}-01$ & $1.22 \mathrm{e}+00$ & \multicolumn{2}{|c|}{0.4043} \\
\hline $1.30 \mathrm{e}-03$ & 2.265 & $2.70 \mathrm{e}-02$ & 1.190 & $4.40 \mathrm{e}-01$ & 1.090 & $1.42 \mathrm{e}-01$ & $1.16 \mathrm{e}+00$ & \multicolumn{2}{|c|}{0.3794} \\
\hline $9.74 \mathrm{e}-04$ & 2.613 & $2.37 \mathrm{e}-02$ & 1.197 & $4.00 \mathrm{e}-01$ & 0.870 & $1.31 \mathrm{e}-01$ & $1.10 \mathrm{e}+00$ & \multicolumn{2}{|c|}{0.3622} \\
\hline $3.24 \mathrm{e}-04$ & 2.658 & $1.39 \mathrm{e}-02$ & 1.130 & $2.25 \mathrm{e}-01$ & 1.002 & $1.00 \mathrm{e}-01$ & $8.46 \mathrm{e}-01$ & \multicolumn{2}{|c|}{0.2654} \\
\hline $2.54 \mathrm{e}-04$ & 2.104 & $1.16 \mathrm{e}-02$ & 1.599 & $2.00 \mathrm{e}-01$ & 1.006 & $9.97 \mathrm{e}-02$ & $8.06 \mathrm{e}-01$ & \multicolumn{2}{|c|}{0.2482} \\
\hline $1.92 \mathrm{e}-04$ & 2.489 & $1.03 \mathrm{e}-02$ & 0.998 & $1.78 \mathrm{e}-01$ & 1.018 & $9.28 \mathrm{e}-02$ & $7.63 \mathrm{e}-01$ & \multicolumn{2}{|c|}{0.2335} \\
\hline $1.68 \mathrm{e}-04$ & 1.162 & $8.72 \mathrm{e}-03$ & 1.506 & $1.59 \mathrm{e}-01$ & 1.031 & $8.74 \mathrm{e}-02$ & $7.26 \mathrm{e}-01$ & \multicolumn{2}{|c|}{0.2188} \\
\hline
\end{tabular}



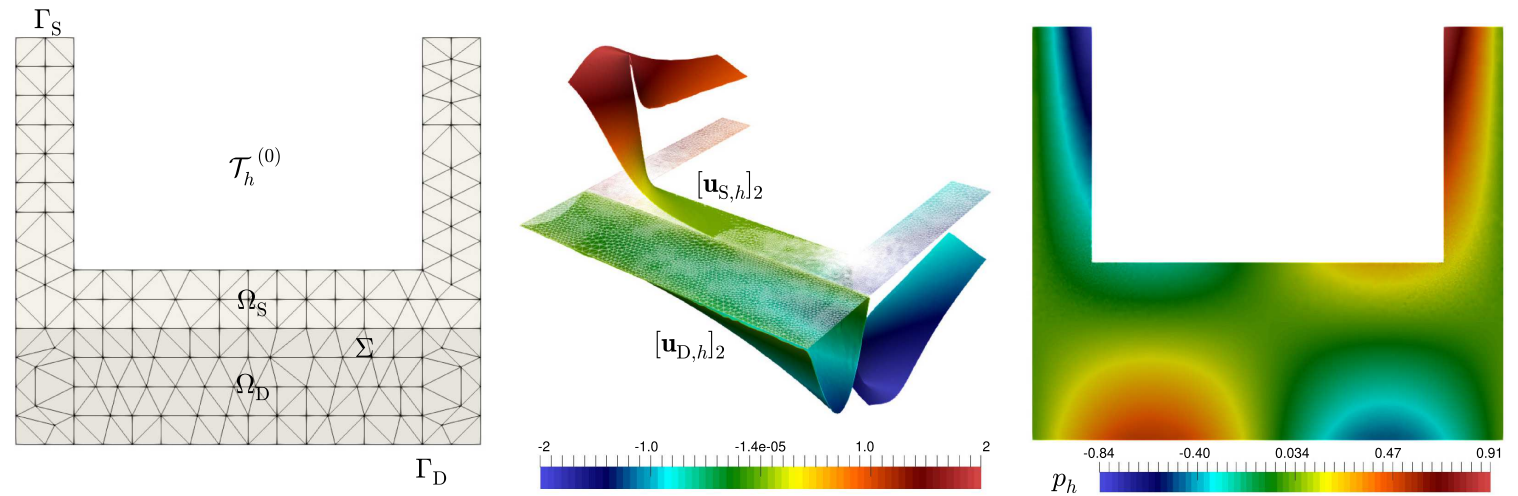

FiguRE 3. Example 2, domain configuration in the initial mesh, second velocity component and pressure in the whole domain.
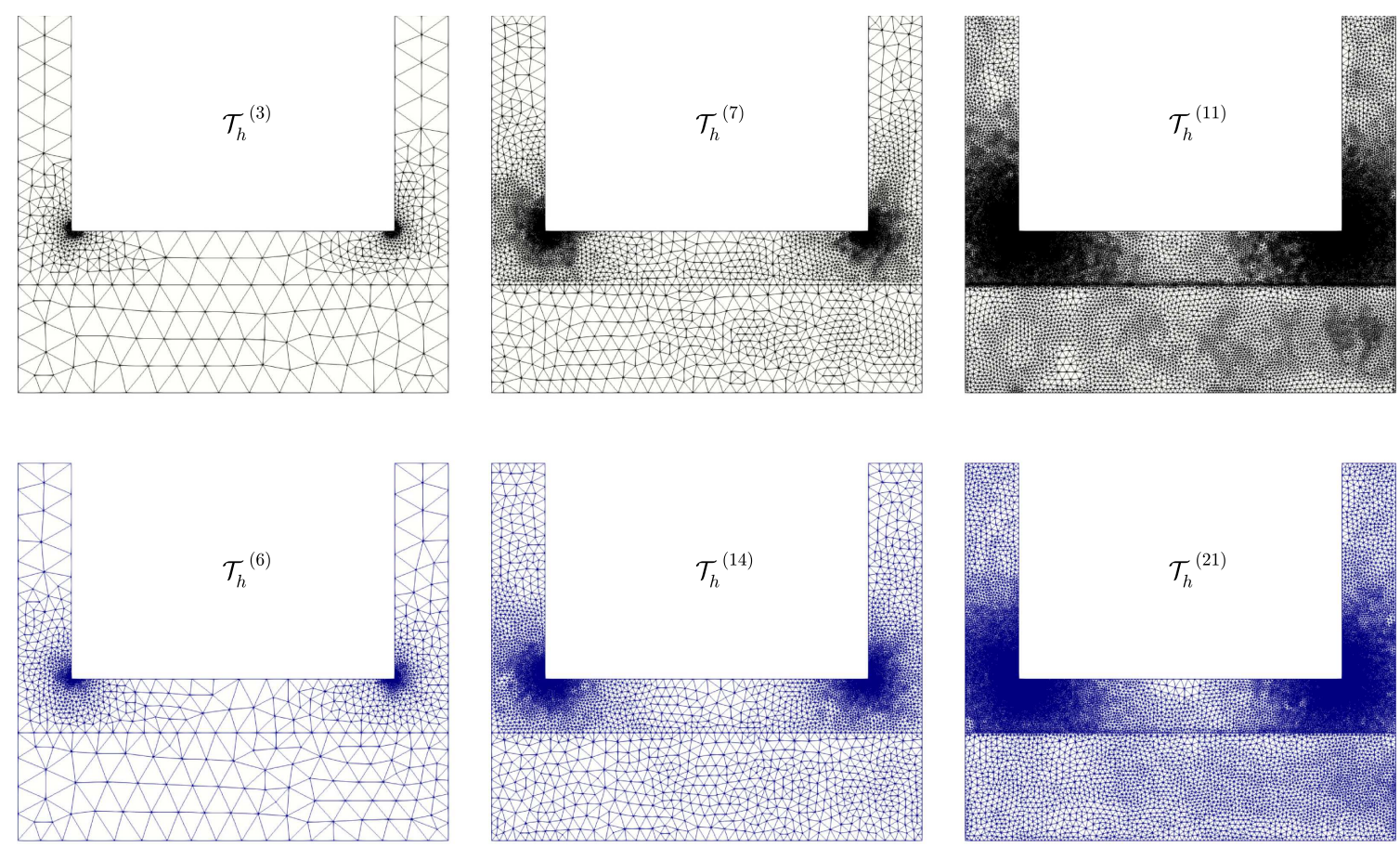

Figure 4. Example 2, three snapshots of adapted meshes according to the indicators $\Theta$ and $\Theta^{1 / 2}$ (top and bottom plots, respectively).

$2 \mathrm{D}$ helmet-shaped domain $\Omega$ is given by the smooth functions

$$
\begin{aligned}
& \mathbf{u}_{S}:=\left(\begin{array}{c}
\frac{\left(x_{2}-0.26\right)}{r_{1}\left(x_{1}, x_{2}\right)}+\frac{\left(x_{2}-0.26\right)}{r_{2}\left(x_{1}, x_{2}\right)} \\
-\frac{\left(x_{1}+0.74\right)}{r_{1}\left(x_{1}, x_{2}\right)}-\frac{\left(x_{1}-0.74\right)}{r_{2}\left(x_{1}, x_{2}\right)}
\end{array}\right), \quad \mathbf{u}_{D}:=\left(\begin{array}{c}
\sin \left(\pi x_{1}\right) \cos \left(\pi x_{1}\right) \exp \left(x_{2}\right) \\
\sin \left(\pi x_{2}\right) \cos \left(\pi x_{2}\right) \exp \left(x_{1}\right)
\end{array}\right), \\
& p_{\star}:=x_{2} \sin \left(\pi x_{1}\right) \quad \text { in } \quad \Omega_{\star}, \quad \text { with } \star \in\{S, D\},
\end{aligned}
$$


where

$$
r_{1}\left(x_{1}, x_{2}\right):=\sqrt{\left(x_{1}+0.74\right)^{2}+\left(x_{2}-0.26\right)^{2}} \quad \text { and } \quad r_{2}\left(x_{1}, x_{2}\right):=\sqrt{\left(x_{1}-0.74\right)^{2}+\left(x_{2}-0.26\right)^{2}} .
$$

Figure 2, summarizes the convergence history of the method applied to a sequence of quasi-uniformly and adaptively refined triangulation of the domain. Sub-optimal rates are observed in the first case, whereas adaptive refinements according to any of the a posteriori error indicators: $\Theta$ and $\Theta^{1 / 2}$, yield optimal convergence. In particular, Table 2 summarizes the errors, rates of convergence, efficiency and reliability indexes, and Newton iterations of the method applied to a sequence of quasi-uniform refinement triangulation of the domain. In turn, Tables 3 and 4 summarizes the convergence history of the primal-mixed scheme after $\Theta$ and $\Theta^{1 / 2}$, respectively, where for the sake of simplicity, we only show twelve rows of a total of twenty two for $\Theta^{1 / 2}$. Notice that in all the examples, when $\Theta<1$ and $h \rightarrow 0$, the rate of convergence of the total error and the efficiency and reliability indexes have the behavior that we expected. Notice also how the adaptive algorithms improves the efficiency of the method by delivering quality solutions at a lower computational cost, to the point that it is possible to get a better one (in terms of $\mathrm{e}(\overrightarrow{\mathbf{u}})$ ) with approximately only the $5.5 \%$ of the degrees of freedom of the last quasi-uniform mesh for the primal-mixed scheme. In addition, and similarly to the first example, we observe that $\mathrm{e}\left(\widehat{\lambda}_{h}\right)$ decreases with order $O(h)$ as well, thus confirming again that $\widehat{\lambda}_{h}$ is well approximated by $\psi_{h}$ on $\partial \Omega_{D}$. On the other hand, in Figure 3 we show the domain configuration in the initial mesh, the second component of velocity and the pressure field in the whole domain obtained through the primal-mixed scheme (via the indicator $\Theta$ ). In particular, we notice that the Navier-Stokes velocity exhibit high gradients near the vertices $(-0.75,0.25)$ and $(0.75,0.25)$. In turn, examples of some adapted meshes generated using $\Theta$ and $\Theta^{1 / 2}$ are collected in Figure 4. We can observe a clear clustering of elements near the vertices in $\Omega_{S}$ of the $2 \mathrm{D}$ helmed-shaped domain as we expected. Notice also a clustering of elements on $\Sigma$.

Acknowledgements. This research was partially supported by ANID-Chile through the project Centro DE Modelamiento Matemático (AFB170001) of the PIA Program: Concurso Apoyo a Centros Científicos y Tecnológicos de Excelencia con Financiamiento Basal, by the project PAI77190084 of the PAI Program: Convocatoria Nacional Subvención a la Instalación en la Academia, and project Fondecyt 1161325; and by Centro de Investigación en Ingeniería Matemática ( $\left.\mathrm{CI}^{2} \mathrm{MA}\right)$, Universidad de Concepción.

\section{REFERENCES}

[1] S. Agmon, Lectures on Elliptic Boundary Value Problems. Van Nostrand, Princeton, NJ (1965).

[2] A. Allendes, E. Otárola and A. Salgado, A posteriori error estimates for the stationary Navier-Stokes equations with Dirac measures. SIAM J. Sci. Comput. 42 (2020) A1860-A1884.

[3] M. Alvarez, G.N. Gatica and R. Ruiz-Baier, A posteriori error analysis for a viscous flow-transport problem. ESAIM: M2AN 50 (2016) 1789-1816.

[4] I. Babuška and G.N. Gatica, A residual-based a posteriori error estimator for the Stokes-Darcy coupled problem. SIAM J. Numer. Anal. 48 (2010) 498-523.

[5] F. Brezzi and M. Fortin, Mixed and Hybrid Finite Element Methods. In: Vol. 15 of Springer Series in Computational Mathematics. Springer-Verlag, New York (1991).

[6] J. Camaño, C. García and R. Oyarzúa, Analysis of a momentum conservative mixed-FEM for the stationary Navier-Stokes problem. To appear in: Numer. Meth. Partial Differ. Equ. (2021).

[7] C. Carstensen, A posteriori error estimate for the mixed finite element method. Math. Comp. 66 (1997) $465-476$.

[8] S. Caucao, D. Mora and R. Oyarzúa, A priori and a posteriori error analysis of a pseudostress-based mixed formulation of the Stokes problem with varying density. IMA J. Numer. Anal. 36 (2016) 947-983.

[9] S. Caucao, G.N. Gatica and R. Oyarzúa, A posteriori error analysis of a fully-mixed formulation for the Navier-Stokes/Darcy coupled problem with nonlinear viscosity. Comput. Methods Appl. Mech. Eng. 315 (2017) 943-971.

[10] S. Caucao, G.N. Gatica, R. Oyarzúa and F. Sandoval, Residual-based A Posteriori Error Analysis for the Coupling of the Navier-Stokes and Darcy-Forchheimer Equations. Centro de Investigación en Ingeniería Matemática $\left(\mathrm{CI}^{2} \mathrm{MA}\right)$, Universidad de Concepción, Chile. Preprint 2019-33 (2019).

[11] S. Caucao, M. Discacciati, G.N. Gatica and R. Oyarzúa, A conforming mixed finite element method for the NavierStokes/Darcy-Forchheimer coupled problem. ESAIM: M2AN 54 (2020) 1689-1723.

[12] P. Clément, Approximation by finite element functions using local regularisation. RAIRO Modél. Math. Anal. Numér. 9 (1975) $77-84$. 
[13] E. Creuse, M. Farhloul and L. Paquet, A posteriori error estimation for the dual mixed finite element method for the $p$-Laplacian in a polygonal domain. Comput. Methods Appl. Mech. Eng. 196 (2007) 2570-2582.

[14] C. Domínguez, G.N. Gatica and S. Meddahi, A posteriori error analysis of a fully-mixed finite element method for a twodimensional fluid-solid interaction problem. J. Comput. Math. 33 (2015) 606-641.

[15] F. Durango and J. Novo, A posteriori error estimations for mixed finite element approximations to the Navier-Stokes equations based on Newton-type linearization. J. Comput. Appl. Math. 367 (2020) 112429.

[16] A. Ern and J.-L. Guermond, Theory and Practice of Finite Elements. In: Vol. 159 of Applied Mathematical Sciences. SpringerVerlag, New York (2004).

[17] V.J. Ervin and T.N. Phillips, Residual a posteriori error estimator for a three-field model of a non-linear generalized Stokes problem. Comput. Methods Appl. Mech. Engrg. 195 (2006) 2599-2610.

[18] M. Farhloul and A.M. Zine, A posteriori error estimation for a dual mixed finite element approximation of non-Newtonian fluid flow problems. Int. J. Numer. Anal. Model. 5 (2008) 320-330.

[19] M. Farhloul, S. Nicaise and L. Paquet, A priori and a posteriori error estimations for the dual mixed finite element method of the Navier-Stokes problem. Numer. Methods Part. Differ. Equ. 25 (2009) 843-869.

[20] G.N. Gatica, A Simple Introduction to the Mixed Finite Element Method. Theory and Applications. Springer Briefs in Mathematics. Springer, Cham (2014).

[21] G.N. Gatica, G.C. Hsiao and S. Meddahi, A residual-based a posteriori error estimator for a two-dimensional fluid-solid interaction problem. Numer. Math. 114 (2009) 63-106.

[22] G.N. Gatica, R. Ruiz-Baier and G. Tierra, A posteriori error analysis of an augmented mixed method for the Navier-Stokes equations with nonlinear viscosity. Comput. Math. Appl. 72 (2016) 2289-2310.

[23] L.F. Gatica, R. Oyarzúa and N. Sánchez, A priori and a posteriori error analysis of an augmented mixed-FEM for the NavierStokes-Brinkman problem. Comput. Math. Appl. 75 (2018) 2420-2444.

[24] V. Girault and P.A. Raviart, Finite Element Methods for Navier-Stokes Equations. Theory and Algorithms. In: Vol. 5 of Springer Series in Computational Mathematics. Springer-Verlag, Berlin (1986).

[25] P. Grisvard, Elliptic Problems in Nonsmooth Domains. In: Vol. 24 of Monographs and Studies in Mathematics. Pitman (Advanced Publishing Program), Boston, MA (1985).

[26] F. Hecht, New development in freefem++. J. Numer. Math. 20 (2012) 251-265.

[27] F. Hecht, Freefem++, 3rd edition, Version 3.58-1. Laboratoire Jacques-Louis Lions, Université Pierre et Marie Curie, Paris (2018). [available in http://www.freefem.org/ff++].

[28] G. Kanschat and D. Schötzau, Energy norm a posteriori error estimation for divergence-free discontinuous Galerkin approximations of the Navier-Stokes equations. Inter. J. Numer. Methods Fluids 57 (2008) 1093-1113.

[29] J.T. Oden, W. Wu and M. Ainsworth, An a posteriori error estimate for finite element approximations of the Navier-Stokes equations. Comput. Methods Appl. Mech. Engrg. 111 (1994) 185-202.

[30] R. Verfürth, A Review of A-Posteriori Error Estimation and Adaptive Mesh-Refinement Techniques. Wiley Teubner, Chichester (1996).

[31] R. Verfürth, A Posteriori Error Estimation Techniques for Finite Element Methods. Numerical Mathematics and Scientific Computation. Oxford University Press, Oxford (2013). 